\title{
Boundary trace of positive solutions of semilinear elliptic equations in Lipschitz domains: the subcritical case
}

\author{
Moshe Marcus And LAURent VERon
}

\begin{abstract}
We study the generalized boundary value problem for nonnegative solutions of $-\Delta u+g(u)=0$ in a bounded Lipschitz domain $\Omega$, when $g$ is continuous and nondecreasing. Using the harmonic measure of $\Omega$, we define a trace in the class of outer regular Borel measures. We amphasize the case where $g(u)=|u|^{q-1} u, q>1$. When $\Omega$ is (locally) a cone with vertex $y$, we prove sharp results of removability and characterization of singular behavior. In the general case, assuming that $\Omega$ possesses a tangent cone at every boundary point and $q$ is subcritical, we prove an existence and uniqueness result for positive solutions with arbitrary boundary trace.
\end{abstract}

Mathematics Subject Classification (2010): 35K60 (primary); 31A20, 31C15, 44A25, 46E35 (secodary).

\section{Introduction}

In this article we study boundary value problems with measure data on the boundary, for equations of the form

$$
-\Delta u+g(u)=0 \text { in } \Omega
$$

where $\Omega$ is a bounded Lipschitz domain in $\mathbb{R}^{N}$ and $g$ is a continuous nondecreasing function vanishing at 0 (in short $g \in \mathcal{G}$ ). A function $u$ is a solution of the equation if $u$ and $g(u)$ belong to $L_{\text {loc }}^{1}(\Omega)$ and the equation holds in the distribution sense. The definition of a solution satisfying a prescribed boundary condition is more complex and will be described later on.

Boundary value problems for (1.1) with measure boundary data in smooth domains (or, more precisely, in $C^{2}$ domains) have been studied intensively in the last 20 years. Much of this work concentrated on the case of power nonlinearities, namely, $g(u)=|u|^{q-1} u$ with $q>1$. For details we address the reader

Both authors were partially sponsored by the French - Israeli cooperation program through grant No. 3-4299. The first author (MM) also wishes to acknowledge the support of the Israeli Science Foundation through grant No. 145-05.

Received December 30, 2009; accepted in revised form August 26, 2010. 
to the following papers and the references therein: Le Gall [17, 18], Dynkin and Kuznetsov [6-8], Mselati [26] (employing in an essential way probabilistic tools) and Marcus and Veron [20-24] (employing purely analytic methods).

The study of the corresponding linear boundary value problem in Lipschitz domains is classical. This study shows that, with a proper interpretation, the basic results known for smooth domains remain valid in the Lipschitz case. Of course there are important differences too: in the Poisson integral formula the Poisson kernel must be replaced by the Martin kernel and, when the boundary data is given by a function in $L^{1}$, the standard surface measure must be replaced by the harmonic measure. The Hopf principle does not hold anymore, but it is partially replaced by the Carleson lemma and the boundary Harnack principledue to Dahlberg [5]. A summary of the basic results for the linear case, to the extent needed in the present work, is presented in Section 2.

One might expect that in the nonlinear case the results valid for smooth domains extend to Lipschitz domains in a similar way. This is indeed the case as long as the boundary data is in $L^{1}$. However, in problems with measure boundary data, we encounter essentially new phenomena.

Following is an overview of our main results on boundary value problems for (1.1).

\section{A. General nonlinearity and finite measure data}

We start with the weak $L^{1}$ formulation of the boundary value problem

$$
-\Delta u+g(u)=0 \text { in } \Omega, u=\mu \text { on } \partial \Omega,
$$

where $\mu \in \mathfrak{M}(\partial \Omega)$.

Let $x_{0}$ be a point in $\Omega$, to be kept fixed, and let $\rho=\rho_{\Omega}$ denote the first eigenfunction of $-\Delta$ in $\Omega$ normalized by $\rho\left(x_{0}\right)=1$. It turns out that the family of test functions appropriate for the boundary value problem is

$$
X(\Omega)=\left\{\eta \in W_{0}^{1,2}(\Omega): \rho^{-1} \Delta \eta \in L^{\infty}(\Omega)\right\} .
$$

If $\eta \in X(\Omega)$ then $\sup |\eta| / \rho<\infty$.

Let $\mathbb{K}[\mu]$ denote the harmonic function in $\Omega$ with boundary trace $\mu$. Then $u$ is an $L^{1}$-weak solution of (1.2) if

$$
u \in L_{\rho}^{1}(\Omega), \quad g(u) \in L_{\rho}^{1}(\Omega)
$$

and

$$
\int_{\Omega}(-u \Delta \eta+g(u) \eta) d x=-\int_{\Omega}(\mathbb{K}[\mu] \Delta \eta) d x \quad \forall \eta \in X(\Omega) .
$$

Note that in (1.5) the boundary data appears only in an implicit form. In the next result we present a more explicit link between the solution and its boundary trace. 
A sequence of domains $\left\{\Omega_{n}\right\}$ is called a Lipschitz exhaustion of $\Omega$ if, for every $n, \Omega_{n}$ is Lipschitz and

$$
\Omega_{n} \subset \bar{\Omega}_{n} \subset \Omega_{n+1}, \quad \Omega=\cup \Omega_{n}, \quad \mathbb{H}_{N-1}\left(\partial \Omega_{n}\right) \rightarrow \mathbb{H}_{N-1}(\partial \Omega) .
$$

In Lischitz domains, the natural way to represent harmonic functions solutions of Dirichlet problems with continuous boundary data is use the harmonic measure. Its definition and mains properties are recalled in Section 2.1. As an illustration of this notion we prove the following:

Proposition 1.1. Let $\left\{\Omega_{n}\right\}$ be an exhaustion of $\Omega$, let $x_{0} \in \Omega_{1}$ and denote by $\omega_{n}$ (respectively $\omega$ ) the harmonic measure on $\partial \Omega_{n}$ (respectively $\partial \Omega$ ) relative to $x_{0}$. If $u$ is an $L^{1}$-weak solution of (1.2) then, for every $Z \in C(\bar{\Omega})$,

$$
\lim _{n \rightarrow \infty} \int_{\partial \Omega_{n}} Z u d \omega_{n}=\int_{\partial \Omega} Z d \mu
$$

We note that any solution of (1.1) is in $W_{\text {loc }}^{1, p}(\Omega)$ for some $p>1$ and consequently possesses an integrable trace on $\partial \Omega_{n}$.

In general problem (1.2) does not possess a solution for every $\mu$. We denote by $\mathfrak{M}^{g}(\partial \Omega)$ the set of measures $\mu \in \mathfrak{M}(\partial \Omega)$ for which such a solution exists. The following statements are established in the same way as in the case of smooth domains:

(i) If a solution exists it is unique. Furthermore the solution depends monotonically on the boundary data.

(ii) If $u$ is an $L^{1}$-weak solution of (1.2) then $|u|$ (respectively $u_{+}$) is a subsolution of this problem with $\mu$ replaced by $|\mu|$ (respectively $\mu_{+}$).

A measure $\mu \in \mathfrak{M}(\partial \Omega)$ is $g$-admissible if $g(\mathbb{K}[|\mu|]) \in L_{\rho}^{1}(\Omega)$. When there is no risk of confusion we shall simply write "admissible" instead of " $g$-admissible". The following provides a sufficient condition for existence.

Theorem 1.2. If $\mu$ is g-admissible then problem (1.2) possesses a unique solution.

B. The boundary trace of positive solutions of (1.1); general nonlinearity

We say that $u \in L_{\text {loc }}^{1}(\Omega)$ is a regular solution of the equation (1.1) if $g(u) \in L_{\rho}^{1}(\Omega)$.

Proposition 1.3. Let $u$ be a positive solution of the equation (1.1). If $u$ is regular then $u \in L_{\rho}^{1}(\Omega)$ and it possesses a boundary trace $\mu \in \mathfrak{M}(\partial \Omega)$. Thus $u$ is the solution of the boundary value problem (1.2) with this measure $\mu$.

As in the case of smooth domains, a positive solution possesses a boundary trace even if the solution is not regular. The boundary trace may be defined in several ways; in every case it is expressed by an unbounded measure. A definition of trace is "good" if the trace uniquely determines the solution. A discussion of the 
various definitions of boundary trace, for boundary value problems in $C^{2}$ domains, with power nonlinearities, can be found in [6,24] and the references therein. In [20] the authors introduced a definition of trace - later referred to as the "rough trace" by Dynkin [6] - which proved to be "good" in the subcritical case, but not in the supercritical case (see [21]). Mselati [26] obtained a "good" definition of trace for the problem with $g(u)=u^{2}$ and $N \geq 4$, in which case this non-linearity is supercritical. His approach employed probabilistic methods developed by Le Gall in a series of papers. For a presentation of these methods we refer the reader to his book [18]. Following this work the authors introduced in [24] a notion of trace, called "the precise trace", defined in the framework of the fine topology associated with the Bessel capacity $C_{2 / q, q^{\prime}}$ on $\partial \Omega$. This definition of trace turned out to be "good" for all power nonlinearities $g(u)=u^{q}, q>1$, at least in the class of $\sigma$ moderate solutions. In the subcritical case, the precise trace reduces to the rough trace. At the same time Dynkin [7] extended Mselati's result to the case $(N+$ $1) /(N-1) \leq q \leq 2$. Finally, Marcus [19] proved that, for $g(u)=u^{q}$ and arbitrary $q \epsilon(N+1)=(N-1)$, every positive solution of $(1.1)$ is $\sigma$-moderate. This result, combined with [24], implies that every positive solution (for any $q>1$ ) is uniquely determined by its precise boundary trace.

In the present paper we confine ourselves to boundary value problems with rough trace data and in the subcritical case (see the definitions below). In a forthcoming paper we shall study equations with power non-linearities in polyhedral domains. In this case we obtain necessary and su cient condi- tions for removability of singular sets [25]. Using these results it is possible to extend the precise trace theory [24] to the case of polyhedral domains.

Here are the main results of the present paper, including the relevant definitions.

Definition 1.4. Let $u$ be a positive supersolution, respectively subsolution, of (1.1). A point $y \in \partial \Omega$ is a regular boundary point relative to $u$ if there exists an open neighborhood $D$ of $y$ such that $g \circ u \in L_{\rho}^{1}(\Omega \cap D)$. If no such neighborhood exists we say that $y$ is a singular boundary point relative to $u$.

The set of regular boundary points of $u$ is denoted by $\mathcal{R}(u)$; its complement on the boundary is denoted by $\mathcal{S}(u)$. Evidently $\mathcal{R}(u)$ is relatively open.

Theorem 1.5. Let $u$ be a positive solution of (1.1) in $\Omega$. Then $u$ possesses a trace on $\mathcal{R}(u)$, given by a Radon measure $v$.

Furthermore, for every compact set $F \subset \mathcal{R}(u)$,

$$
\int_{\Omega}(-u \Delta \eta+g(u) \eta) d x=-\int_{\Omega}\left(\mathbb{K}\left[v \chi_{F}\right] \Delta \eta\right) d x
$$

for every $\eta \in X(\Omega)$ such that $\operatorname{supp} \eta \cap \partial \Omega \subset F$ and $\nu \chi_{F} \in \mathfrak{M}^{g}(\partial \Omega)$.

Definition 1.6. Let $g \in \mathcal{G}$. Let $u$ be a positive solution of (1.1) with regular boundary set $\mathcal{R}(u)$ and singular boundary set $\mathcal{S}(u)$. The Radon measure $v$ in $\mathcal{R}(u)$ associated with $u$ as in Theorem 1.5 is called the regular part of the trace of $u$. The couple 
$(\nu, \mathcal{S}(u))$ is called the boundary trace of $u$ on $\partial \Omega$. This trace is also represented by the (possibly unbounded) Borel measure $\bar{v}$ given by

$$
\bar{v}(E)= \begin{cases}v(E), & \text { if } E \subset \mathcal{R}(u) \\ \infty, & \text { otherwise }\end{cases}
$$

The boundary trace of $u$ in the sense of this definition will be denoted by $\operatorname{tr}_{\partial \Omega} u$.

Let

$$
V_{v}:=\sup \left\{u_{\nu_{\chi_{F}}}: F \subset \mathcal{R}(u), F \text { compact }\right\}
$$

where $u_{\nu \chi_{F}}$ denotes the solution of (1.2) with $\mu=v \chi_{F}$. Then $V_{v}$ is called the semi-regular component of $u$.

Definition 1.7. A compact set $F \subset \partial \Omega$ is removable relative to (1.1) if the only non-negative solution $u \in C(\bar{\Omega} \backslash F)$ which vanishes on $\bar{\Omega} \backslash F$ is the trivial solution $u=0$.

An important subclass of $\mathcal{G}$ is the class of functions $g$ satisfying the KellerOsserman condition, that is

$$
\int_{a}^{\infty} \frac{d s}{\sqrt{G(s)}}<\infty \quad \text { where } G(s)=\int_{0}^{s} g(\tau) d \tau,
$$

for some $a>0$. It is proved in $[16,27]$ that, if $g$ satisfies this condition, there exists a non-increasing function $h$ from $\mathbb{R}_{+}$to $\mathbb{R}_{+}$with limits

$$
\lim _{s \rightarrow 0} h(s)=\infty \quad \lim _{s \rightarrow \infty} h(s)=a_{+}:=\inf \{a>0: g(a)>0\}
$$

such that any solution $u$ of (1.1) satisfies

$$
u(x) \leq h(\operatorname{dist}(x, \partial \Omega)) \quad \forall x \in \Omega .
$$

Lemma 1.8. Let $g \in \mathcal{G}$ and assume that $g$ satisfies the Keller-Osserman condition. Let $F \subset \partial \Omega$ be a compact set and denote by $\mathcal{U}_{F}$ the class of solutions $u$ of (1.1) which satisfy the condition,

$$
u \in C(\bar{\Omega} \backslash F), \quad u=0 \text { on } \partial \Omega \backslash F .
$$

Then there exists a function $U_{F} \in \mathcal{U}_{F}$ such that

$$
u \leq U_{F} \quad \forall u \in \mathcal{U}_{F} .
$$

Furthermore, $\mathcal{S}\left(U_{F}\right)=: F^{\prime} \subset F ; F^{\prime}$ need not be equal to $F$.

Definition 1.9. $U_{F}$ is called the maximal solution associated with $F$. The set $F^{\prime}=$ $\mathcal{S}\left(U_{F}\right)$ is called the $g$-kernel of $F$ and denoted by $k_{g}(F)$. 
Theorem 1.10. Let $g \in \mathcal{G}$ and assume that $g$ is convex and satisfies the KellerOsserman condition.

EXISTENCE. The following set of conditions is necessary and sufficient for existence of a solution $u$ of the generalized boundary value problem

$$
-\Delta u+g(u)=0 \text { in } \Omega, \quad \operatorname{tr}_{\partial \Omega} u=(\nu, F),
$$

where $F \subset \partial \Omega$ is a compact set and $\nu$ is a Radon measure on $\partial \Omega \backslash F$.

(i) For every compact set $E \subset \partial \Omega \backslash F, v \chi_{E} \in \mathfrak{M}^{g}(\partial \Omega)$.

(ii) If $k_{g}(F)=F^{\prime}$, then $F \backslash F^{\prime} \subset \mathcal{S}\left(V_{v}\right)$.

When this holds,

$$
V_{v} \leq u \leq V_{v}+U_{F} .
$$

Furthermore if $F$ is a removable set then (1.2) possesses exactly one solution.

UNIQUENESS. Given a compact set $F \subset \partial \Omega$, assume that

$$
U_{E} \text { is the unique positive solution with trace }\left(0, k_{g}(E)\right)
$$

for every compact $E \subset F$. Under this assumption:

(a) If $u$ is a solution of (1.15) then

$$
\max \left(V_{v}, U_{F}\right) \leq u \leq V_{v}+U_{F} .
$$

(b) Equation (1.1) possesses at most one solution satisfying (1.18).

(c) Condition (1.17) is necessary and sufficient in order that (1.15) possess at most one solution.

MONOTONICITY.

(d) Let $u_{1}, u_{2}$ be two positive solutions of (1.1) with boundary traces $\left(v_{1}, F_{1}\right)$ and $\left(v_{2}, F_{2}\right)$ respectively. Suppose that $F_{1} \subset F_{2}$ and that $v_{1} \leq v_{2} \chi_{F_{1}}=: v_{2}^{\prime}$. If (1.17) holds for $F=F_{2}$ then $u_{1} \leq u_{2}$.

In the remaining part of this paper we consider equation (1.1) with power nonlinearity:

$$
-\Delta u+|u|^{q-1} u=0
$$

with $q>1$.

C. Classification of positive solutions in a conical domain possessing an isolated singularity at the vertex

Let $C_{S}$ be a cone with vertex 0 and opening $S \subset S^{N-1}$, where $S$ is a Lipschitz domain. Put $\Omega=C_{S} \cap B_{1}(0)$. Denote by $\lambda_{S}$ the first eigenvalue and by $\phi_{S}$ the first eigenfunction of $-\Delta^{\prime}$ in $W_{0}^{1,2}(S)$ normalized by $\max \phi_{S}=1$. Put

$$
\alpha_{S}=\frac{1}{2}\left(N-2+\sqrt{(N-2)^{2}+4 \lambda_{S}}\right)
$$


and

$$
\Phi_{1}(x)=\frac{1}{\gamma}|x|^{-\alpha_{S}} \phi_{S}(x /|x|)
$$

where $\gamma_{S}$ is a positive number. $\Phi_{1}$ is a harmonic function in $C_{S}$ vanishing on $\partial C_{S} \backslash\{0\}$ and $\gamma$ is chosen so that the boundary trace of $\Phi_{1}$ is $\delta_{0}$ (=Dirac measure on $\partial C_{S}$ with mass 1 at the origin). Further denote $\Omega_{S}=C_{S} \cap B_{1}(0)$.

It was shown in [9] that, if $q \geq 1+\frac{2}{\alpha_{S}}$ there is no solution of (1.19) in $\Omega$ with isolated singularity at 0 . We obtain the following result.

Theorem 1.11. Assume that $1<q<1+\frac{2}{\alpha_{S}}$. Then $\delta_{0}$ is admissible for $\Omega$ and consequently, for every real $k$, there exists a unique solution of this equation in $\Omega$ with boundary trace $k \delta_{0}$. This solution, denoted by $u_{k}$ satisfies

$$
u_{k}(x)=k \Phi_{1}(x)(1+o(1)) \quad \text { as } x \rightarrow 0 .
$$

The function

$$
u_{\infty}=\lim _{k \rightarrow \infty} u_{k}
$$

is the unique positive solution of (5.1) in $\Omega_{S}$ which vanishes on $\partial \Omega \backslash\{0\}$ and is strongly singular at 0 , i.e.,

$$
\int_{\Omega} u_{\infty}^{q} \rho d x=\infty
$$

where $\rho$ is the first eigenfunction of $-\Delta$ in $\Omega$ normalized by $\rho\left(x_{0}\right)=1$ for some (fixed) $x_{0} \in \Omega$. Its asymptotic behavior at 0 is given by,

$$
u_{\infty}(x)=|x|^{-\frac{2}{q-1}} \omega_{S}(x /|x|)(1+o(1)) \quad \text { as } x \rightarrow 0
$$

where $\omega$ is the (unique) positive solution of

$$
-\Delta^{\prime} \omega-\lambda_{N, q} \omega+|\omega|^{q-1} \omega=0
$$

on $S^{N-1}$ with

$$
\lambda_{N, q}=\frac{2}{q-1}\left(\frac{2 q}{q-1}-N\right) .
$$

As a consequence one can state the following classification result.

Theorem 1.12. Assume that $1<q<q_{S}=1+2 / \alpha_{S}$ and denote

$$
\tilde{\alpha}_{S}=\frac{1}{2}\left(2-N+\sqrt{(N-2)^{2}+4 \lambda_{S}}\right) .
$$

If $u \in C\left(\bar{\Omega}_{S} \backslash\{0\}\right)$ is a positive solution of (1.19) vanishing on $\left(\partial C_{S} \cap B_{r_{0}}(0)\right) \backslash\{0\}$, the following alternative holds:

Either

$$
\limsup _{x \rightarrow 0}|x|^{-\tilde{\alpha}_{S}} u(x)<\infty
$$


or

$$
\text { there exist } k>0 \text { such that (1.20) holds, }
$$

or

(1.22) holds.

In the first case $u \in C(\bar{\Omega})$; in the second, $u$ possesses a weak singularity at the vertex while in the last case $u$ has a strong singularity there.

\section{Criticality in Lipschitz domains}

Let $\Omega$ be a Lipschitz domain and let $\xi \in \partial \Omega$. We say that $q_{\xi}$ is the critical value for (1.19) at $\xi$ if, for $1<q<q_{\xi}$, the equation possesses a solution with boundary trace $\delta_{\xi}$ while, for $q>q_{\xi}$ no such solution exists. We say that $q_{\xi}^{\sharp}$ is the secondary critical value at $\xi$ if for $1<q<q_{\xi}^{\sharp}$ there exists a non-trivial solution of (1.19) which vanishes on $\partial \Omega \backslash\{\xi\}$ but for $q>q_{\xi}^{\sharp}$ no such solution exists. Thus, if $q_{\xi}<q<q_{\xi}^{\sharp}$ there exist solutions with isolated singularity at $\xi$ but these solutions do not possess a nite boundary trace.

In the case of smooth domains, $q_{\xi}=q_{\xi}^{\sharp}$ and $q_{\xi}=(N+1) /(N-1)$ for every boundary point $\xi$. Furthermore, if $q=q_{\xi}$ there is no solution with isolated singularity at $\xi$, i.e., an isolated singularity at $\xi$ is removable.

In Lipschitz domains the critical value depends on the point. Clearly $q_{\xi} \leq q_{\xi}^{\sharp}$, but the question whether, in general, $q_{\xi}=q_{\xi}^{\sharp}$ remains open. However we prove that, if $\Omega$ is a polyhedron, $q_{\xi}=q_{\xi}^{\sharp}$ at every point and the function $\xi \rightarrow q_{\xi}$ obtains only a finite number of values. In fact it is constant on each open face and each open edge, of any dimension. In addition, if $q=q_{\xi}$, an isolated singularity at $\xi$ is removable. The same holds true in a piecewise $C^{2}$ domain $\Omega$ except that $\xi \rightarrow q_{\xi}$ is not constant on edges but it is continuous on every relatively open edge.

For general Lipschitz domains, we can provide only a partial answer to the question posed above.

We say that $\Omega$ possesses a tangent cone at a point $\xi \in \partial \Omega$ if the limiting inner cone with vertex at $\xi$ is the same as the limiting outer cone at $\xi$.

Theorem 1.13. Suppose that $\Omega$ possesses a tangent cone $C_{\xi}^{\Omega}$ at a point $\xi \in \partial \Omega$ and denote by $q_{c, \xi}$ the critical value for this cone at the vertex $\xi$. Then

$$
q_{\xi}=q_{\xi}^{\sharp}=q_{c, \xi}
$$

Furthermore, if $1<q<q_{\xi}$ then $\delta_{\xi}$ is admissible, i.e.,

$$
M_{\xi}:=\int_{\Omega} K(x, \xi)^{q} \rho(x) d x<\infty .
$$

We do not know if, under the assumptions of this theorem, an isolated singularity at $\xi$ is removable when $q=q_{c, \xi}$. It would be useful to resolve this question. 
E. The generalized boundary value problem in Lipschitz domains: the subcritical case

In the case of smooth domains, a boundary value problem for equation (1.19) is either subcritical or supercritical. This is no longer the case when the domain is merely Lipschitz since the criticality varies from point to point. In this part of the paper we discuss the generalized boundary value problem in the strictly subcritical case.

Under the conditions of Theorem 1.13 we know that, if $\xi \in \partial \Omega$ and $1<$ $q<q_{\xi}$ then $K(\cdot, \xi) \in L_{\rho}^{1}(\Omega)$. In the next result, we derive, under an additional restriction on $q$, uniform estimates of the norm $\|K(\cdot, \xi)\|_{L_{\rho}^{1}(\Omega)}$. Such estimates are needed in the study of existence and uniqueness. For its statement we need the following notation:

If $z \in \partial \Omega$, we denote by $S_{z, r}$ the opening of the largest cone $C_{S}$ with vertex at $z$ such that $C_{S} \cap B_{r}(z) \subset \Omega \cup\{z\}$. If $E$ is a compact subset of $\partial \Omega$ we denote:

$$
q_{E}^{*}=\lim _{r \rightarrow 0}\left(\left\{q_{S_{z, r}}: z \in \partial \Omega, \operatorname{dist}(z, E)<r\right\}\right) .
$$

We observe that

$$
q_{E}^{*} \leq \inf \left\{q_{c, z}: z \in E\right\}
$$

but this number also measures, in a sense, the rate of convergence of interior cones to the limiting cones. If $\Omega$ is convex then $q_{E}^{*} \leq(N+1) /(N-1)$ for every nonempty set $E$. On the other hand if $\Omega$ is the complement of a bounded convex set then $q_{E}^{*}=(N+1) /(N-1)$.

Theorem 1.14. If $E$ is a compact subset of $\partial \Omega$ and $1<q<q_{E}^{*}$ then, there exists $M>0$ such that

$$
\int_{\Omega} K^{q}(x, y) \rho(x) d x \leq M \quad \forall y \in E .
$$

Using this theorem we obtain:

Theorem 1.15. Assume that $\Omega$ is a bounded Lipschitz domain and $u$ is a positive solution of (1.19). If $y \in \mathcal{S}(u)$ (i.e. $y \in \partial \Omega$ is a singular point of $u$ ) and $1<q<$ $q_{\{y\}}^{*}$ then, for every $k>0$, the measure $k \delta_{y}$ is admissible and

$$
u \geq u_{k \delta_{y}}=\text { solution with boundary trace } k \delta_{y} .
$$

Remark 1.16. It can be shown that, if $q>q_{\{y\}}^{*},(1.26)$ may not hold. For instance, such solutions exist if $\Omega$ is a smooth, obtuse cone and $y$ is the vertex of the cone. Therefore the condition $q<q_{\{y\}}^{*}$ for every $y \in \partial \Omega$ is, in some sense necessary for uniqueness in the subcritical case.

As a consequence we first obtain the existence and uniqueness result in the context of bounded measures. 
Theorem 1.17. Let $E \subset \partial \Omega$ be a closed set and assume that $1<q<q_{E}^{*}$. Then, for every $\mu \in \mathfrak{M}(\Omega)$ such that $\operatorname{supp} \mu \subset E$ there exists a (unique) solution $u_{\mu}$ of (5.1) in $\Omega$ with boundary trace $\mu$.

Further, using Theorems 1.10, 1.11 and 1.14, we establish the existence and uniqueness result for generalized problems.

Theorem 1.18. Let $\Omega$ be a bounded Lipschitz domain which possesses a tangent cone at every boundary point. If

$$
1<q<q_{\partial \Omega}^{*}
$$

then, for every positive, outer regular Borel measure $\bar{v}$ on $\partial \Omega$, there exists a unique solution $u$ of (1.19) such that $\operatorname{tr}_{\partial \Omega}(u)=\bar{v}$.

\section{Boundary value problems}

\subsection{Classical harmonic analysis in Lipschitz domains}

A bounded domain $\Omega \subset \mathbb{R}^{N}$ is called a Lipschitz domain if there exist positive numbers $r_{0}, \lambda_{0}$ and a cylinder

$$
O_{r_{0}}=\left\{\xi=\left(\xi_{1}, \xi^{\prime}\right) \in \mathbb{R}^{N}:\left|\xi^{\prime}\right|<r_{0},\left|\xi_{1}\right|<r_{0}\right\}
$$

such that, for every $y \in \partial \Omega$ there exist:

(i) A Lipschitz function $\psi^{y}$ on the $(N-1)$-dimensional ball $B_{r_{0}}^{\prime}(0)$ with Lipschitz constant $\geq \lambda_{0}$

(ii) An isometry $T^{y}$ of $\mathbb{R}^{N}$ such that

$$
\begin{aligned}
& T^{y}(y)=0, \quad\left(T^{y}\right)^{-1}\left(O_{r_{0}}\right):=O_{r_{0}}^{y}, \\
& T^{y}\left(\partial \Omega \cap O_{r_{0}}^{y}\right)=\left\{\left(\psi^{y}\left(\xi^{\prime}\right), \xi^{\prime}\right): \xi^{\prime} \in B_{r_{0}}^{\prime}(0)\right\} \\
& T^{y}\left(\Omega \cap O_{r_{0}}^{y}\right)=\left\{\left(\xi_{1}, \xi^{\prime}\right): \xi^{\prime} \in B_{r_{0}}^{\prime}(0),-r_{0}<\xi_{1}<\psi^{y}\left(\xi^{\prime}\right)\right\} .
\end{aligned}
$$

The constant $r_{0}$ is called a localization constant of $\Omega ; \lambda_{0}$ is called a Lipschitz constant of $\Omega$. The pair $\left(r_{0}, \lambda_{0}\right)$ is called a Lipschitz character (or, briefly, L-character) of $\Omega$. Note that, if $\Omega$ has L-character $\left(r_{0}, \lambda_{0}\right)$ and $r^{\prime} \in\left(0, r_{0}\right), \lambda^{\prime} \in\left(\lambda_{0}, \infty\right)$ then $\left(r^{\prime}, \lambda^{\prime}\right)$ is also an L-character of $\Omega$.

By the Rademacher theorem, the outward normal unit vector exists $\mathcal{H}^{N-1}$-a.e. on $\partial \Omega$, where $\mathcal{H}^{N-1}$ is the $\mathrm{N}-1$ dimensional Hausdorff measure. The unit normal at a point $y \in \partial \Omega$ will be denoted by $\mathbf{n}_{y}$.

We list below some facts concerning the Dirichlet problem in Lipschitz domains. 
A.1- Let $x_{0} \in \Omega, h \in C(\partial \Omega)$ and denote $L_{x_{0}}(h):=v_{h}\left(x_{0}\right)$ where $v_{h}$ is the solution of the Dirichlet problem

$$
\begin{cases}-\Delta v=0 & \in \Omega \\ v=h & \text { on } \partial \Omega .\end{cases}
$$

Then $L_{x_{0}}$ is a continuous linear functional on $C(\partial \Omega)$. Therefore there exists a unique Borel measure on $\partial \Omega$, called the harmonic measure in $\Omega$, denoted by $\omega_{\Omega}^{x_{0}}$ such that

$$
v_{h}\left(x_{0}\right)=\int_{\partial \Omega} h d \omega_{\Omega}^{x_{0}} \quad \forall h \in C(\partial \Omega) .
$$

When there is no danger of confusion, the subscript $\Omega$ will be dropped. Because of Harnack's inequality the measures $\omega^{x_{0}}$ and $\omega^{x}, x_{0}, x \in \Omega$ are mutually absolutely continuous. For every fixed $x \in \Omega$ denote the Radon-Nikodym derivative by

$$
K(x, y):=\frac{d \omega^{x}}{d \omega^{x_{0}}}(y) \text { for } \omega^{x_{0}} \text {-a.e. } y \in \partial \Omega .
$$

Then, for every $\bar{x} \in \Omega$, the function $y \mapsto K(\bar{x}, y)$ is positive and continuous on $\partial \Omega$ and, for every $\bar{y} \in \partial \Omega$, the function $x \mapsto K(x, \bar{y})$ is harmonic in $\Omega$ and satisfies

$$
\lim _{x \rightarrow y} K(x, \bar{y})=0 \quad \forall y \in \partial \Omega \backslash\{\bar{y}\} .
$$

By [12]

$$
\lim _{z \rightarrow y} \frac{G(x, z)}{G\left(x_{0}, z\right)}=K(x, y) \quad \forall y \in \partial \Omega .
$$

Thus the kernel $K$ defined above is the Martin kernel.

The following is an equivalent definition of the harmonic measure [12]:

For any closed set $E \subset \partial \Omega$

$$
\begin{aligned}
& \omega^{x_{0}}(E) \\
& :=\inf \left\{\phi\left(x_{0}\right): \phi \in C(\Omega)_{+} \text {superharmonic in } \Omega, \liminf _{x \rightarrow E} \phi(x) \geq 1\right\} .
\end{aligned}
$$

The extension to open sets and then to arbitrary Borel sets is standard.

By (2.4), (2.5) and (2.7), the unique solution $v$ of (2.3) is given by

$$
\begin{aligned}
& v(x)=\int_{\partial \Omega} K(x, y) h(y) d \omega^{x_{0}}(y) \\
& =\inf \left\{\phi \in C(\Omega): \phi \text { superharmonic, } \liminf _{x \rightarrow y} \phi(x) \geq h(y), \quad \forall y \in \partial \Omega\right\} .
\end{aligned}
$$

For details see [12].

A.2- Let $\left(x_{0}, y_{0}\right) \in \Omega \times \partial \Omega$. A function $v$ defined in $\Omega$ is called a kernel function at $y_{0}$ if it is positive and harmonic in $\Omega$ and verifies $v\left(x_{0}\right)=1$ and $\lim _{x \rightarrow y} v(x)=0$ for any $y \in \partial \Omega \backslash\left\{y_{0}\right\}$. It is proved in [12, Section 3] that the kernel function at $y_{0}$ is unique. Clearly this unique function is $K\left(\cdot, y_{0}\right)$. 
A.3- We denote by $G(x, y)$ the Green kernel for the Laplacian in $\Omega \times \Omega$. This means that the solution of the Dirichlet problem

$$
\begin{cases}-\Delta u=f & \text { in } \Omega \\ u=0 & \text { on } \partial \Omega\end{cases}
$$

with $f \in C^{2}(\bar{\Omega})$, is expressed by

$$
u(x)=\int_{\Omega} G(x, y) f(y) d y \quad \forall y \in \bar{\Omega} .
$$

We shall write $(2.10)$ as $u=\mathbb{G}[f]$.

A.4- Let $\Lambda$ be the first eigenvalue of $-\Delta$ in $W_{0}^{1,2}(\Omega)$ and denote by $\rho$ the corresponding eigenfunction normalized by $\max _{\Omega} \rho=1$.

Let $0<\delta<\operatorname{dist}\left(x_{0}, \Omega\right)$ and put

$$
C_{x_{0}, \delta}:=\max _{\left|x-x_{0}\right|=\delta} G\left(x, x_{0}\right) / \rho(x) .
$$

Since $C_{x_{0}, \delta} \rho-G\left(\cdot, x_{0}\right)$ is superharmonic, the maximum principle implies that

$$
0 \leq G\left(x, x_{0}\right) \leq C_{x_{0}, \delta} \rho(x) \quad \forall x \in \Omega \backslash B_{\delta}\left(x_{0}\right) .
$$

On the other hand, by [15, Lemma 3.4]: for any $x_{0} \in \Omega$ there exists a constant $C_{x_{0}}>0$ such that

$$
0 \leq \rho(x) \leq C_{x_{0}} G\left(x, x_{0}\right) \quad \forall x \in \Omega .
$$

A.5- For every bounded regular Borel measure $\mu$ on $\partial \Omega$ the function

$$
v(x)=\int_{\partial \Omega} K(x, y) d \mu(y) \quad \forall x \in \Omega,
$$

is harmonic in $\Omega$. We denote this relation by $v=\mathbb{K}[\mu]$.

A.6- Conversely, for every positive harmonic function $v$ in $\Omega$ there exists a unique positive bounded regular Borel measure $\mu$ on $\partial \Omega$ such that (2.13) holds. The measure $\mu$ is constructed as follows [12, Theorem 4.3].

Let $S P(\Omega)$ denote the set of continuous, non-negative superharmonic functions in $\Omega$. Let $v$ be a positive harmonic function in $\Omega$.

If $E$ denotes a relatively closed subset of $\Omega$, denote by $R_{v}^{E}$ the function defined in $\Omega$ by

$$
R_{v}^{E}(x)=\inf \{\phi(x): \phi \in S P(\Omega), \phi \geq v \text { in } E\} .
$$

Then $R_{v}^{E}$ is superharmonic in $\Omega, R_{v}^{E}$ decreases as $E$ decreases and, if $F$ is another relatively closed subset of $\Omega$, then

$$
R_{v}^{E \cup F} \leq R_{v}^{E}+R_{v}^{F} .
$$


Now, relative to a point $x \in \Omega$, the measure $\mu$ is defined by

$$
\mu_{v}^{x}(F)=\inf \left\{R_{v}^{E}(x): E=\bar{D} \cap \Omega, D \text { open in } \mathbb{R}^{N}, D \supset F\right\},
$$

for every compact set $F \subset \partial \Omega$. From here it is extended to open sets and then to arbitrary Borel sets in the usual way.

It is easy to see that, if $D$ contains $\partial \Omega$ then $R_{v}^{\bar{D} \cap \Omega}=v$. Therefore

$$
\mu_{v}^{x}(\partial \Omega)=v(x) .
$$

In addition, if $F$ is a compact subset of the boundary, the function $x \mapsto \mu_{v}^{x}(F)$ is harmonic in $\Omega$ and vanishes on $\partial \Omega \backslash F$.

A.7- If $x, x_{0}$ are two points in $\Omega$, the Harnack inequality implies that $\mu_{v}^{x}$ is absolutely continuous with respect to $\mu_{v}^{x_{0}}$. Therefore, for $\mu_{v}^{x_{0}}$-a.e. point $y \in \partial \Omega$, the density function $d \mu_{v}^{x} / d \mu_{v}^{x_{0}}(y)$ is a kernel function at $y$. By the uniqueness of the kernel function it follows that

$$
\frac{d \mu_{v}^{x}}{d \mu_{v}^{x_{0}}}(y)=K(x, y), \quad \mu_{v}^{x_{0}} \text {-a.e. } y \in \partial \Omega .
$$

Therefore, using (2.15),

$$
\begin{aligned}
& \text { (a) } \mu_{v}^{x}(F)=\int_{F} K(x, y) d \mu^{x_{0}}(y), \\
& \text { (b) } v(x)=\int_{\partial \Omega} K(x, y) d \mu^{x_{0}}(y) .
\end{aligned}
$$

A.8- By a result of Dahlberg [5, Theorem 3], the (interior) normal derivative of $G\left(\cdot, x_{0}\right)$ exists $\mathcal{H}_{N-1}$-a.e. on $\partial \Omega$ and is positive. In addition, for every Borel set $E \subset \partial \Omega$

$$
\omega^{x_{0}}(E)=\gamma_{N} \int_{E} \partial G\left(\xi, x_{0}\right) / \partial \mathbf{n}_{\xi} d S_{\xi},
$$

where $\gamma_{N}(N-2)$ is the surface area of the unit ball in $\mathbb{R}^{N}$ and $d S$ is surface measure on $\partial \Omega$. Thus, for each fixed $x \in \Omega$, the harmonic measure $\omega^{x}$ is absolutely continuous relative to $\left.\mathcal{H}_{N-1}\right|_{\partial \Omega}$ with density function $P(x, \cdot)$ given by

$$
P(x, \xi)=\partial G(\xi, x) / \partial \mathbf{n}_{\xi} \text { for a.e. } \xi \in \partial \Omega .
$$

In view of (2.8), the unique solution $v$ of (2.3) is given by

$$
v(x)=\int_{\Omega} P(x, \xi) h(\xi) d S_{\xi}
$$

for every $h \in C(\partial \Omega)$. Accordingly $P$ is the Poisson kernel for $\Omega$. The expression on the right hand side of (2.20) will be denoted by $\mathbb{P}[h]$. We observe that,

$$
\mathbb{K}\left[h \omega^{x_{0}}\right]=\mathbb{P}[h] \quad \forall h \in C(\partial \Omega) .
$$


A.9- The boundary Harnack principle, first proved in [5], can be formulated as follows [13].

Let $D$ be a Lipschitz domain with L-character $\left(r_{0}, \lambda_{0}\right)$. Let $\xi \in \partial D$ and $\delta \in$ $\left(0, r_{0}\right)$. Assume that $u, v$ are positive harmonic functions in $D$, vanishing on $\partial D \cap$ $B_{\delta}(\xi)$. Then there exists a constant $C=C\left(N, r_{0}, \lambda_{0}\right)$ such that

$$
C^{-1} u(x) / v(x) \leq u(y) / v(y) \leq C u(x) / v(x) \quad \forall x, y \in B_{\delta / 2}(\xi) .
$$

A.10- Let $D, D^{\prime}$ be two Lipschitz domains with L-character $\left(r_{0}, \lambda_{0}\right)$. Assume that $D^{\prime} \subset D$ and $\partial D \cap \partial D^{\prime}$ contains a relatively open set $\Gamma$. Let $x_{0} \in D^{\prime}$ and let $\omega, \omega^{\prime}$ denote the harmonic measures of $D, D^{\prime}$ respectively, relative to $x_{0}$. Then, for every compact set $F \subset \Gamma$, there exists a constant $c_{F}=C\left(F, N, r_{0}, \lambda_{0}, x_{0}\right)$ such that

$$
\omega^{\prime}\left\lfloor_{F} \leq \omega L_{F} \leq c_{F} \omega^{\prime}\left\lfloor_{F}\right.\right.
$$

Indeed, if $G, G^{\prime}$ denote the Green functions of $D, D^{\prime}$ respectively then, by the boundary Harnack principle,

$$
\partial G^{\prime}\left(\xi, x_{0}\right) / \partial \mathbf{n}_{\xi} \leq \partial G\left(\xi, x_{0}\right) / \partial \mathbf{n}_{\xi} \leq c_{F} \partial G\left(\xi, x_{0}\right) / \partial \mathbf{n}_{\xi} \text { for a.e. } \xi \in F .
$$

Therefore (2.23) follows from (2.18).

A.11- By [15, Lemma 3.3], for every positive harmonic function $v$ in $\Omega$,

$$
\int_{\Omega} v(x) G\left(x, x_{0}\right) d x<\infty .
$$

In view of (2.12), it follows that $v \in L_{\rho}^{1}(\Omega)$.

\subsection{The dynamic approach to boundary trace}

Let $\Omega$ be a bounded Lipschitz domain and $\left\{\Omega_{n}\right\}$ be a Lipschitz exhaustion of $\Omega$. This means that, for every $n, \Omega_{n}$ is Lipschitz and

$$
\Omega_{n} \subset \bar{\Omega}_{n} \subset \Omega_{n+1}, \quad \Omega=\cup \Omega_{n}, \quad \mathbb{H}_{N-1}\left(\partial \Omega_{n}\right) \rightarrow \mathbb{H}_{N-1}(\partial \Omega) .
$$

Lemma 2.1. Let $x_{0} \in \Omega_{1}$ and denote by $\omega_{n}$ (respectively $\omega$ ) the harmonic measure in $\Omega_{n}$ (respectively $\Omega$ ) relative to $x_{0}$. Then, for every $Z \in C(\bar{\Omega})$,

$$
\lim _{n \rightarrow \infty} \int_{\partial \Omega_{n}} Z d \omega_{n}=\int_{\partial \Omega} Z d \omega .
$$

Proof. By the definition of harmonic measure

$$
\int_{\partial \Omega_{n}} d \omega_{n}=1
$$


We extend $\omega_{n}$ as a Borel measure on $\bar{\Omega}$ by setting $\omega_{n}\left(\bar{\Omega} \backslash \partial \Omega_{n}\right)=0$, and keep the notation $\omega_{n}$ for the extension. Since the sequence $\left\{\omega_{n}\right\}$ is bounded, there exists a weakly convergent subsequence (still denoted by $\left\{\omega_{n}\right\}$ ). Evidently the limiting measure, say $\tilde{\omega}$, is supported in $\partial \Omega$ and $\tilde{\omega}(\partial \Omega)=1$. It follows that for every $Z \in C(\bar{\Omega})$,

$$
\int_{\partial \Omega_{n}} Z d \omega_{n} \rightarrow \int_{\partial \Omega} Z d \tilde{\omega}
$$

Let $\zeta:=\left.Z\right|_{\partial \Omega}$ and $z:=\mathbb{K}^{\Omega}[\zeta]$. Again by the definition of harmonic measure,

$$
\int_{\partial \Omega_{n}} z d \omega_{n}=\int_{\partial \Omega} \zeta d \omega=z\left(x_{0}\right)
$$

It follows that

$$
\int_{\partial \Omega} \zeta d \tilde{\omega}=\int_{\partial \Omega} \zeta d \omega
$$

for every $\zeta \in C(\partial \Omega)$. Consequently $\tilde{\omega}=\omega$. Since the limit does not depend on the subsequence it follows that the whole sequence $\left\{\omega_{n}\right\}$ converges weakly to $\omega$. This implies (2.27).

In the next lemma we continue to use the notation introduced above.

Lemma 2.2. Let $x_{0} \in \Omega_{1}$, let $\mu$ be a bounded Borel measure on $\partial \Omega$ and put $v:=$ $\mathbb{K}^{\Omega}[\mu]$. Then, for every $Z \in C(\bar{\Omega})$,

$$
\lim _{n \rightarrow \infty} \int_{\partial \Omega_{n}} Z v d \omega_{n}=\int_{\partial \Omega} Z d \mu
$$

Proof. It is sufficient to prove the result for positive $\mu$. Let $h_{n}:=\left.v\right|_{\partial \Omega_{n}}$. Evidently $v=\mathbb{K}^{\Omega_{n}}\left[h_{n} \omega_{n}\right]$ in $\Omega_{n}$. Therefore

$$
v\left(x_{0}\right)=\int_{\partial \Omega_{n}} h_{n} d \omega_{n}=\mu(\partial \Omega) .
$$

Let $\mu_{n}$ denote the extension of $h_{n} \omega_{n}$ as a measure in $\bar{\Omega}$ such that $\mu_{n}\left(\bar{\Omega} \backslash \partial \Omega_{n}\right)=0$. Then $\left\{\mu_{n}\right\}$ is bounded and consequently there exists a weakly convergent subsequence $\left\{\mu_{n_{j}}\right\}$. The limiting measure, say $\tilde{\mu}$, is supported in $\partial \Omega$ and

$$
\tilde{\mu}(\partial \Omega)=v\left(x_{0}\right)=\mu(\partial \Omega) .
$$

It follows that for every $Z \in C(\bar{\Omega})$,

$$
\int_{\partial \Omega_{n_{j}}} Z d \mu_{n_{j}} \rightarrow \int_{\partial \Omega} Z d \tilde{\mu} .
$$

To complete the proof, we have to show that $\tilde{\mu}=\mu$. Let $F$ be a closed subset of $\partial \Omega$ and put,

$$
\mu^{F}=\mu \chi_{F}, \quad v^{F}=\mathbb{K}^{\Omega}\left[\mu^{F}\right] .
$$


Let $h_{n}^{F}:=\left.v^{F}\right|_{\partial \Omega_{n}}$ and let $\mu_{n}^{F}$ denote the extension of $h_{n}^{F} \omega_{n}$ as a measure in $\bar{\Omega}$ such that $\mu_{n}^{F}\left(\bar{\Omega} \backslash \partial \Omega_{n}\right)=0$. As in the previous part of the proof, there exists a weakly convergent subsequence of $\left\{\mu_{n_{j}}^{F}\right\}$. The limiting measure $\tilde{\mu}^{F}$ is supported in $F$ and

$$
\tilde{\mu}^{F}(F)=\tilde{\mu}^{F}(\partial \Omega)=v^{F}\left(x_{0}\right)=\mu^{F}(\partial \Omega)=\mu(F) .
$$

As $v^{F} \leq v$, we have $\tilde{\mu}^{F} \leq \tilde{\mu}$. Consequently

$$
\mu(F) \leq \tilde{\mu}(F)
$$

Observe that $\tilde{\mu}$ depends on the first subsequence $\left\{\mu_{n_{j}}\right\}$, but not on the second subsequence. Therefore (2.30) holds for every closed set $F \subset \partial \Omega$, which implies that $\mu \leq \tilde{\mu}$. On the other hand, $\mu$ and $\tilde{\mu}$ are positive measures which, by (2.29), have the same total mass. Therefore $\mu=\tilde{\mu}$.

Lemma 2.3. Let $\mu \in \mathfrak{M}(\partial \Omega)$ (= space of bounded Borel measures on $\partial \Omega$ ). Then $\mathbb{K}[\mu] \in L_{\rho}^{1}(\Omega)$ and there exists a constant $C=C(\Omega)$ such that

$$
\|\mathbb{K}[\mu]\|_{L_{\rho}^{1}(\Omega)} \leq C\|\mu\|_{\mathfrak{M}(\partial \Omega)} .
$$

In particular if $h \in L^{1}(\partial \Omega ; \omega)$ then

$$
\|\mathbb{P}[h]\|_{L_{\rho}^{1}(\Omega)} \leq C\|h\|_{L^{1}(\partial \Omega ; \omega)} .
$$

Proof. Let $x_{0}$ be a point in $\Omega$ and let $K$ be defined as in (2.5). Put $\phi(\cdot)=G\left(\cdot, x_{0}\right)$ and $d_{0}=\operatorname{dist}\left(x_{0}, \Omega\right)$. Let $\left(r_{0}, \lambda_{0}\right)$ denote the Lipschitz character of $\Omega$.

By [3, Theorem 1], there exist positive constants $c_{1}\left(N, r_{0}, \lambda_{0}, d_{0}\right)$ and $c_{0}(N$, $\left.r_{0}, \lambda_{0}, d_{0}\right)$ such that for every $y \in \partial \Omega$,

$$
c_{1}^{-1} \frac{\phi(x)}{\phi^{2}\left(x^{\prime}\right)}|x-y|^{2-N} \leq K(x, y) \leq c_{1} \frac{\phi(x)}{\phi^{2}\left(x^{\prime}\right)}|x-y|^{2-N},
$$

for all $x, x^{\prime} \in \Omega$ such that

$$
c_{0}|x-y|<\operatorname{dist}\left(x^{\prime}, \partial \Omega\right) \leq\left|x^{\prime}-y\right|<|x-y|<\frac{1}{4} \min \left(d_{0}, r_{0} / 8\right) .
$$

Therefore, by (2.12) and (2.11), there exists a constant $c_{2}\left(N, r_{0}, \lambda_{0}, d_{0}\right)$ such that

$$
c_{2}^{-1} \frac{\phi^{2}(x)}{\phi^{2}\left(x^{\prime}\right)}|x-y|^{2-N} \leq \rho(x) K(x, y) \leq c_{2} \frac{\phi^{2}(x)}{\phi^{2}\left(x^{\prime}\right)}|x-y|^{2-N}
$$

for $x, x^{\prime}$ as above. There exists a constant $\bar{c}_{0}$, depending on $c_{0}, N$, such that, for every $x \in \Omega$ satisfying $|x-y|<\frac{1}{4} \min \left(d_{0}, r_{0} / 8\right)$ there exists $x^{\prime} \in \Omega$ which satisfies (2.34) and also

$$
\left|x-x^{\prime}\right| \leq \bar{c}_{0} \min \left(\operatorname{dist}(x, \partial \Omega), \operatorname{dist}\left(x^{\prime}, \partial \Omega\right)\right)
$$


By the Harnack chain argument, $\phi(x) / \phi\left(x^{\prime}\right)$ is bounded by a constant depending on $N, \bar{c}_{0}$. Therefore

$$
c_{3}^{-1}|x-y|^{2-N} \leq \rho(x) K(x, y) \leq c_{3}|x-y|^{2-N}
$$

for some constant $c_{3}\left(N, r_{0}, \lambda_{0}, d_{0}\right)$ and all $x \in \Omega$ sufficiently close to the boundary. Assuming that $\mu \geq 0$,

$$
\int_{\Omega} \mathbb{K}[\mu](x) \rho(x) d x=\int_{\partial \Omega} \int_{\Omega} K(x, \xi) \rho(x) d x d \mu(\xi) \leq C\|\mu\|_{\mathfrak{M}(\partial \Omega)} .
$$

In the general case we apply this estimate to $\mu_{+}$and $\mu_{-}$. This implies (2.31). For the last statement of the theorem see (2.21).

Definition 2.4. Let $D$ be a Lipschitz domain and let $\left\{D_{n}\right\}$ be a Lipschitz exhaustion of $D$. We say that $\left\{D_{n}\right\}$ is a uniform Lipschitz exhaustion if there exist positive numbers $\bar{r}, \bar{\lambda}$ such that $D_{n}$ has L-character $(\bar{r}, \bar{\lambda})$ for all $n \in \mathbb{N}$. The pair $(\bar{r}, \bar{\lambda})$ is an L-character of the exhaustion.

Lemma 2.5. Assume $D, D^{\prime}$ are two Lipschitz domains such that

$$
\Gamma \subset \partial D \cap \partial D^{\prime} \subset \partial\left(D \cup D^{\prime}\right)
$$

where $\Gamma$ is a relatively open set. Suppose $D, D^{\prime}, D \cup D^{\prime}$ have L-character $\left(r_{0}, \lambda_{0}\right)$. Let $x_{0}$ be a point in $D \cap D^{\prime}$ and put

$$
d_{0}=\min \left(\operatorname{dist}\left(x_{0}, \partial D\right), \operatorname{dist}\left(x_{0}, \partial D^{\prime}\right)\right) .
$$

Let $u$ be a positive harmonic function in $D \cup D^{\prime}$ and denote its boundary trace on $D$ (respectively $\left.D^{\prime}\right)$ by $\mu$ (respectively $\left.\mu^{\prime}\right)$. Then, for every compact set $F \subset \Gamma$, there exists a constant $c_{F}=c\left(F, r_{0}, \lambda_{0}, d_{0}, N\right)$ such that

$$
c_{F}^{-1} \mu^{\prime}\left\lfloor_{F} \leq \mu\left\lfloor_{F} \leq c_{F} \mu^{\prime}\left\lfloor_{F} .\right.\right.\right.
$$

Proof. We prove (2.36) in the case that $D^{\prime} \subset D$. This implies (2.36) in the general case by comparison of the boundary trace on $\partial D$ or $\partial D^{\prime}$ with the boundary trace on $\partial\left(D \cup D^{\prime}\right)$.

Let $Q$ be an open set such that $Q \cap D$ is Lipschitz and

$$
F \subset Q, \quad \bar{Q} \cap D \subset D^{\prime}, \quad \bar{Q} \cap \partial D \subset \Gamma .
$$

Then there exist uniform Lipschitz exhaustions of $D$ and $D^{\prime}$, say $\left\{D_{n}\right\}$ and $\left\{D_{n}^{\prime}\right\}$, possessing the following properties:

(i) $\bar{D}_{n}^{\prime} \cap Q=\bar{D}_{n} \cap Q$.

(ii) $x_{0} \in D_{1}^{\prime}$ and dist $\left(x_{0}, \partial D_{1}^{\prime}\right) \geq \frac{1}{4} d_{0}$. 
(iii) There exist $r_{Q}>0$ and $\lambda_{Q}>0$ such that both exhaustions have L-character $\left(r_{Q}, \lambda_{Q}\right)$.

Put $\Gamma_{n}:=\partial D_{n} \cap Q=\partial D_{n}^{\prime} \cap Q$ and let $\omega_{n}$ (respectively $\omega_{n}^{\prime}$ ) denote the harmonic measure, relative to $x_{0}$, of $D_{n}$ (respectively $D_{n}^{\prime}$ ). By Lemma 2.2,

$$
\int_{\Gamma_{n}} \phi u(y) d \omega_{n}(y) \rightarrow \int_{\Gamma} \phi d \mu,
$$

and

$$
\int_{\Gamma_{n}} \phi u(y) d \omega_{n}^{\prime}(y) \rightarrow \int_{\Gamma} \phi d \mu^{\prime}
$$

for every $\phi \in C_{c}(Q)$. By $\mathbf{A . 1 0}$ there exists a constant $c_{Q}=c\left(Q, r_{Q}, \lambda_{Q}, d_{0}, N\right)$ such that

$$
\omega_{n}^{\prime} \mathrm{L}_{n} \leq \omega_{n} \mathrm{~L}_{\Gamma_{n}} \leq c_{Q} \omega_{n}^{\prime} \mathrm{L}_{\Gamma_{n}} .
$$

This implies (2.36).

\section{3. $L^{1}$ data}

We denote by $X(\Omega)$ the space of test functions,

$$
X(\Omega)=\left\{\eta \in W_{0}^{1,2}(\Omega): \rho^{-1} \Delta \eta \in L^{\infty}(\Omega)\right\} .
$$

Let $X_{+}(\Omega)$ denote its positive cone.

Let $f \in L^{\infty}(\Omega)$, and let $u$ be the weak $W_{0}^{1,2}$ solution of the Dirichlet problem

$$
-\Delta u=f \text { in } \Omega, \quad u=0 \text { on } \partial \Omega .
$$

If $\Omega$ is a Lipschitz domain (as we assume here) then $u \in C(\bar{\Omega})$ (see [28]). Since $\mathbb{G}[f]$ is a weak $W_{0}^{1,2}$ solution, it follows that the solution of (2.38), which is unique in $C(\bar{\Omega})$, is given by $u=\mathbb{G}[f]$. If, in addition, $|f| \leq c_{1} \rho$ then, by the maximum principle,

$$
|u| \leq\left(c_{1} / \Lambda\right) \rho,
$$

where $\Lambda$ is the first eigenvalue of $-\Delta$ in $\Omega$.

In particular, if $\eta \in X(\Omega)$ then $\eta \in C(\bar{\Omega})$ and it satisfies

$$
\begin{aligned}
-\mathbb{G}[\Delta \eta] & =\eta, \\
|\eta| & \leq \Lambda^{-1}\left\|\rho^{-1} \Delta \eta\right\|_{L^{\infty}} \rho .
\end{aligned}
$$

If, in addition, $\Omega$ is a $C^{2}$ domain then the solution of (2.38) is in $C^{1}(\bar{\Omega})$. 
Lemma 2.6. Let $\Omega$ be a Lipschitz bounded domain. Then for any $f \in L_{\rho}^{1}(\Omega)$ there exists a unique $u \in L_{\rho}^{1}(\Omega)$ such that

$$
-\int_{\Omega} u \Delta \eta d x=\int_{\Omega} f \eta d x \quad \forall \eta \in X(\Omega) .
$$

Furthermore $u=\mathbb{G}[f]$. Conversely, if $f \in L_{\text {loc }}^{1}(\Omega), f \geq 0$ and there exists $x_{0} \in \Omega$ such that $\mathbb{G}[f]\left(x_{0}\right)<\infty$ then $f \in L_{\rho}^{1}(\Omega)$. Finally

$$
\|u\|_{L_{\rho}(\Omega)} \leq \Lambda^{-1}\|f\|_{L_{\rho}(\Omega)} .
$$

Proof. First assume that $f$ is bounded. We have already observed that, in this case, the weak $W_{0}^{1,2}$ solution $u$ of the Dirichlet problem $(2.38)$ is in $C(\bar{\Omega})$ and $u=\mathbb{G}[f]$. Furthermore, it follows from [4] that

$$
\int_{\Omega} \nabla \eta \cdot \nabla u d x=-\int_{\Omega} u \Delta \eta d x .
$$

Thus $u=\mathbb{G}[f]$ is also a weak $L_{\rho}^{1}$ solution (in the sense of (2.42)).

Let $\eta_{0}$ be the weak $W_{0}^{1,2}$ solution of (2.38) when $f=\operatorname{sgn}(u) \rho$; evidently $\eta_{0} \in X(\Omega)$. If $u \in L_{\rho}^{1}(\Omega)$ is a solution of (2.42) for some $f \in L_{\rho}^{1}(\Omega)$ then

$$
\int_{\Omega}|u| \rho d x=\int_{\Omega} f \eta_{0} d x \leq \Lambda^{-1} \int_{\Omega}|f| \rho d x .
$$

The second inequality follows from (2.39). This proves (2.43) and implies uniqueness.

Now assume that $f \in L_{\rho}^{1}(\Omega)$ and let $\left\{f_{n}\right\}$ be a sequence of bounded functions such that $f_{n} \rightarrow f$ in this space. Let $u_{n}$ be the weak $W_{0}^{1,2}$ solution of (2.38) with $f$ replaced by $f_{n}$. Then $u_{n}$ satisfies (2.42) and $u_{n}=\mathbb{G}\left[f_{n}\right]$. By (2.43), $\left\{u_{n}\right\}$ converges in $L_{\rho}^{1}(\Omega)$, say $u_{n} \rightarrow u$. In view of (2.11) it follows that $u=\mathbb{G}[f]$ and that $u$ satisfies (2.42).

If $f \in L_{\mathrm{loc}}^{1}(\Omega), f \geq 0$ and $\mathbb{G}[f]\left(x_{0}\right)<\infty$ then, by (2.12), $f \in L_{\rho}^{1}(\Omega)$.

Lemma 2.7. Let $\Omega$ be a Lipschitz bounded domain. If $f \in L_{\rho}^{1}(\Omega)$ and $h \in$ $L^{1}(\partial \Omega ; \omega)$, there exists a unique $u \in L_{\rho}^{1}(\Omega)$ satisfying

$$
\int_{\Omega}(-u \Delta \eta-f \eta) d x=-\int_{\Omega} \mathbb{P}[h] \Delta \eta d x \quad \forall \eta \in X(\Omega)
$$

or equivalently

$$
u=\mathbb{G}[f]-\mathbb{P}[h] .
$$


The following estimate holds

$$
\begin{aligned}
\|u\|_{L_{\rho}^{1}(\Omega)} & \leq c\left(\|f\|_{L_{\rho}^{1}(\Omega)}+\|\mathbb{P}[h]\|_{L_{\rho}^{1}(\Omega)}\right) \\
& \leq c\left(\|f\|_{L_{\rho}^{1}(\Omega)}+\|h\|_{L^{1}(\partial \Omega, \omega)}\right) .
\end{aligned}
$$

Furthermore, for any nonnegative element $\eta \in X(\Omega)$, we have

$$
-\int_{\Omega}|u| \Delta \eta d x \leq-\int_{\Omega} \mathbb{P}[|h|] \Delta \eta d x+\int_{\Omega} \eta f \operatorname{sgn}(u) d x,
$$

and

$$
-\int_{\Omega} u_{+} \Delta \eta d x \leq-\int_{\Omega} \mathbb{P}\left[h_{+}\right] \Delta \eta d x+\int_{\Omega} \eta f \operatorname{sgn}_{+}(u) d x .
$$

Proof. Existence. By Lemma 2.3, the assumption on $h$ implies that $\mathbb{P}[|h|] \in L_{\rho}^{1}(\Omega)$. If we denote by $v$ the unique function in $L_{\rho}^{1}(\Omega)$ which satifies

$$
-\int_{\Omega} v \Delta \eta d x=-\int_{\Omega} f \eta d x \quad \forall \eta \in X(\Omega),
$$

then $u=v-\mathbb{P}[h] \in L_{\rho}^{1}(\Omega)$ and (2.45) holds.

By Lemma 2.6, (2.46) is equivalent to (2.45).

Estimate (2.47). This inequality follows from (2.45) and (2.43).

Estimate (2.49). Let $\left\{\Omega_{n}\right\}$ be an exhaustion of $\Omega$ by smooth domains. If $u$ is the solution of (2.45) and $h_{n}:=\left.u\right|_{\partial \Omega_{n}}$ then, in $\Omega_{n}$,

$$
u=\mathbb{G}^{\Omega_{n}}[f]-\mathbb{P}^{\Omega_{n}}\left[h_{n}\right] \text { in } \Omega_{n},
$$

or equivalently,

$$
\begin{aligned}
\int_{\Omega_{n}}(-u \Delta \eta-f \eta) d x & =-\int_{\Omega_{n}} \mathbb{P}\left[h_{n}\right] \Delta \eta d x \\
& =-\int_{\partial \Omega_{n}}(\partial \eta / \partial \mathbf{n}) h_{n} d x \quad \forall \eta \in X\left(\Omega_{n}\right) .
\end{aligned}
$$

We recall that, since $\Omega_{n}$ is smooth, $\eta \in X\left(\Omega_{n}\right)$ implies that $\eta \in C^{1}\left(\bar{\Omega}_{n}\right)$. In addition it is known that (see e.g. [29]), for every non-negative $\eta \in X\left(\Omega_{n}\right)$,

$$
\int_{\Omega_{n}}(-|u| \Delta \eta-f \eta \operatorname{sign} u) d x \leq-\int_{\partial \Omega_{n}} \partial \eta / \partial \mathbf{n}\left|h_{n}\right| d x .
$$

Let $\rho_{n}$ be the first eigenfunction of $-\Delta$ in $\Omega_{n}$, normalized by $\rho_{n}(\bar{x})=1$ for some $\bar{x} \in \Omega_{1}$. Let $\eta$ be a non-negative function in $X(\Omega)$ and let $\eta_{n}$ be the solution of the problem

$$
\Delta z=(\Delta \eta) \rho_{n} / \rho \text { in } \Omega_{n}, z=0 \text { on } \partial \Omega_{n} .
$$


Then $\eta_{n} \in X\left(\Omega_{n}\right)$ and, since $\rho_{n} \rightarrow \rho$,

$$
\Delta \eta_{n} \rightarrow \Delta \eta, \quad \eta_{n} \rightarrow \eta .
$$

If $v:=\mathbb{P}[|h|]$ then $v \geq|u|$ so that

$$
\tilde{h}_{n}:=\left.v\right|_{\partial \Omega_{n}} \geq\left|h_{n}\right|
$$

Therefore

$$
\begin{aligned}
& -\int_{\partial \Omega_{n}} \partial \eta_{n} / \partial \mathbf{n}\left|h_{n}\right| d x \leq-\int_{\partial \Omega_{n}} \partial \eta / \partial \mathbf{n}\left|\tilde{h}_{n}\right| d x= \\
& -\int_{\Omega_{n}} \mathbb{P}^{\Omega_{n}}\left[\tilde{h}_{n}\right] \Delta \eta_{n} d x=-\int_{\Omega_{n}} v \Delta \eta_{n} d x \rightarrow-\int_{\Omega} v \Delta \eta d x .
\end{aligned}
$$

Finally, (2.51) and (2.52) imply (2.48).

Estimate (2.49). This inequality is obtained by adding (2.45) and (2.48).

Definition 2.8. We shall say that a function $g: \mathbb{R} \rightarrow \mathbb{R}$ belongs to $\mathcal{G}(\mathbb{R})$ if it is continuous, nondecreasing and $g(0)=0$.

Lemma 2.9. Let $\Omega$ be a Lipschitz bounded domain and $g \in \mathcal{G}(\mathbb{R})$. If $f \in L_{\rho}^{1}(\Omega)$ and $h \in L^{1}(\partial \Omega ; \omega)$, there exists a unique $u \in L_{\rho}^{1}(\Omega)$ such that $g(u) \in L_{\rho}^{1}(\Omega)$ and

$$
\int_{\Omega}(-u \Delta \eta+(g(u)-f) \eta) d x=-\int_{\Omega} \mathbb{P}[h] \Delta \eta d x \quad \forall \eta \in X(\Omega) .
$$

The correspondence $(f, h) \mapsto u$ is increasing.

If $u, u^{\prime}$ are solutions of (2.53) corresponding to data $f, h$ and $f^{\prime}, h^{\prime}$ respectively then the following estimate holds:

$$
\begin{aligned}
& \left\|u-u^{\prime}\right\|_{L_{\rho}^{1}(\Omega)}+\left\|g(u)-g\left(u^{\prime}\right)\right\|_{L_{\rho}^{1}(\Omega)} \\
& \leq c\left(\left\|f-f^{\prime}\right\|_{L_{\rho}^{1}(\Omega)}+\left\|\mathbb{P}\left[h-h^{\prime}\right]\right\|_{L_{\rho}^{1}(\Omega)}\right) \\
& \leq c\left(\left\|f-f^{\prime}\right\|_{L_{\rho}^{1}(\Omega)}+\left\|h-h^{\prime}\right\|_{L^{1}(\partial \Omega, \omega)}\right) .
\end{aligned}
$$

Finally, for any nonnegative element $\eta \in X(\Omega)$, we have

$$
-\int_{\Omega}|u| \Delta \eta d x+\int_{\Omega}|g(u)| \eta d x \leq-\int_{\Omega} \mathbb{P}[|h|] \Delta \eta d x+\int_{\Omega} \eta f \operatorname{sgn}(u) d x,
$$

and

$$
-\int_{\Omega} u_{+} \Delta \eta d x+\int_{\Omega} g(u)_{+} \eta d x \leq-\int_{\Omega} \mathbb{P}\left[h_{+}\right] \Delta \eta d x+\int_{\Omega} \eta f \operatorname{sgn}_{+}(u) d x .
$$


Proof. If $u, u^{\prime}$ are two solutions as stated above then $v=u-u^{\prime}$ satisfies

$$
\int_{\Omega}(-v \Delta \eta+F \eta) d x=-\int_{\Omega} \mathbb{P}\left[h-h^{\prime}\right] \Delta h d x \quad \forall \eta \in X(\Omega)
$$

where $F=g(u)-g\left(u^{\prime}\right)-\left(f-f^{\prime}\right) \in L_{\rho}^{1}(\Omega)$. Applying (2.48) to this equation and using the properties of $g$ described in Definition 2.8 we obtain (2.54). Similarly we obtain (2.55) and (2.56), using (2.48) and (2.49). These inequalities imply uniqueness and monotone dependence on data.

In the case that $f$ and $h$ are bounded, existence is obtained by the standard variational method. In general we approach $f$ in $L_{\rho}^{1}(\Omega)$ by functions in $C_{c}^{\infty}(\Omega)$ and $h$ in $L^{1}(\partial \Omega ; \omega)$ by functions in $C(\partial \Omega)$ and employ (2.54).

\section{Measure data}

Denote by $\mathfrak{M}_{\rho}(\Omega)$ the space of Radon measures $v$ in $\Omega$ such that $\rho|\nu|$ is a bounded measure.

Lemma 3.1. Let $\Omega$ be a Lipschitz bounded domain. Let $v \in \mathfrak{M}_{\rho}(\Omega)$ and $u \in$ $L_{\text {loc }}^{1}(\Omega)$ be a nonnegative solution of

$$
-\Delta u=v \quad \text { in } \Omega
$$

Then $u \in L_{\rho}^{1}(\Omega)$ and there exists a unique positive Radon measure $\mu$ on $\partial \Omega$ such that

$$
u=\mathbb{K}[\mu]+\mathbb{G}[v]
$$

Proof. Let $D$ be a smooth subdomain of $\Omega$ such that $\bar{D} \subset \Omega$. Since $u \in W_{\text {loc }}^{1, p}(\Omega)$ for some $p>1$ it follows that $u$ possesses a trace, say $h_{D}$, in $W^{1-\frac{1}{p}, p}(\partial D)$. Put $v:=u-\mathbb{G}^{D}[v]$. Then $-\Delta v=0$ in $D$ and $v \geq 0$ on $\partial D$ and therefore in $D$. If $\left\{D_{n}\right\}$ is an increasing sequence of such domains, converging to $\Omega$, then $\mathbb{G}^{D_{n}}[\nu] \uparrow \mathbb{G}^{\Omega}[\nu]$. Thus $v=u-\mathbb{G}^{\Omega}[\nu]$ is a non-negative harmonic function in $\Omega$ and consequently possesses a boundary trace $\mu \in \mathfrak{M}(\partial \Omega)$ such that $v=\mathbb{K}[\mu]$.

Lemma 3.2. Let $\Omega$ be a Lipschitz bounded domain. If $\nu \in \mathfrak{M}_{\rho}(\Omega)$ and $\mu \in$ $\mathfrak{M}(\partial \Omega)$, there exists a unique $u \in L_{\rho}^{1}(\Omega)$ satisfying

$$
\int_{\Omega}-u \Delta \eta d x=\int_{\Omega} \eta d v-\int_{\Omega} \mathbb{K}[\mu] \Delta \eta d x \quad \forall \eta \in X(\Omega) .
$$

This is equivalent to

$$
u=\mathbb{G}[\nu]+\mathbb{K}[\mu] .
$$


The following estimate holds

$$
\begin{aligned}
\|u\|_{L_{\rho}^{1}(\Omega)} & \leq c\left(\|v\|_{\mathfrak{M}_{\rho}(\Omega)}+\|\mathbb{K}[\mu]\|_{L_{\rho}^{1}(\Omega)}\right) \\
& \leq c\left(\|v\|_{\mathfrak{M}_{\rho}(\Omega)}+\|\mu\|_{\mathfrak{M}(\partial \Omega)}\right) .
\end{aligned}
$$

In addition, if $d v=f d x$ for some $f \in L_{\rho}^{1}(\Omega)$ then, for any nonnegative element $\eta \in X(\Omega)$, we have

$$
-\int_{\Omega}|u| \Delta \eta d x \leq-\int_{\Omega} \mathbb{K}[|\mu|] \Delta \eta d x+\int_{\Omega} \eta f \operatorname{sgn}(u) d x,
$$

and

$$
-\int_{\Omega} u_{+} \Delta \eta d x \leq-\int_{\Omega} \mathbb{K}\left[\mu_{+}\right] \Delta \eta d x+\int_{\Omega} \eta f \operatorname{sgn}_{+}(u) d x .
$$

Proof. We approximate $\mu$ by a sequence $\left\{h_{n} P\left(x_{0}, \cdot\right)\right\}$ and $v$ by a sequence $\left\{f_{n}\right\}$ such that

$$
h_{n} P\left(x_{0}, \cdot\right) \in L^{1}(\partial \Omega), \quad h_{n} P\left(x_{0}, \cdot\right) \mathcal{H}_{N-1} \rightarrow \mu \quad \text { weakly in measure }
$$

and

$$
f_{n} \in L_{\rho}^{1}(\Omega), \quad f_{n} \rightarrow v \quad \text { weakly relative to } C_{\rho}(\Omega),
$$

where $C_{\rho}$ denotes the space of functions $\zeta \in C(\Omega)$ such that $\rho \zeta \in L^{\infty}(\Omega)$. Applying Lemma 2.7 to problem (2.47) $\left(f, h\right.$ replaced by $\left.f_{n}, h_{n}\right)$ and taking the limit we obtain a solution $u \in L_{\rho}^{1}(\Omega)$ of (3.2) satisfying (3.4).

Lemma 2.6 implies that any solution $u$ of (3.2) satisfies (3.3). Therefore the solution is unique and hence (3.4) holds for all solutions.

Inequalities (3.5) and (3.6) are proved in the same way as the corresponding inequalities in Lemma 2.7

Definition 3.3. Let $\Omega$ be a bounded Lipschitz domain and let $g \in \mathcal{G}(\mathbb{R})$. If $\mu \in$ $\mathfrak{M}(\partial \Omega)$, a function $u \in L_{\rho}^{1}(\Omega)$ is a weak solution of

$$
\begin{cases}-\Delta u+g(u)=0 & \text { in } \Omega \\ u=\mu & \text { in } \partial \Omega\end{cases}
$$

if $g(u) \in L_{\rho}^{1}(\Omega)$ and

$$
u+\mathbb{G}[g(u)]=\mathbb{K}[\mu]
$$

a.e. in $\Omega$. Equivalently

$$
\int_{\Omega}(-u \Delta \eta+g(u) \eta) d x=-\int_{\Omega}(\mathbb{K}[\mu] \Delta \eta) d x \quad \forall \eta \in X(\Omega) .
$$

The measure $\mu$ is called the boundary trace of $u$ on $\partial \Omega$. 
Similarly a function $u \in L_{\rho}^{1}(\Omega)$ is a weak supersolution, respectively subsolution, of (3.7) if $g(u) \in L_{\rho}^{1}(\Omega)$ and

$$
u+\mathbb{G}[g(u)] \geq \mathbb{K}[\mu] \text { respectively } u+\mathbb{G}[g(u)] \leq \mathbb{K}[\mu] .
$$

This is equivalent to (3.9), with $=$ replaced by $\geq$ or $\leq$, holding for every positive $\eta \in X(\Omega)$.

Remark 3.4. It follows from this definition and Lemma 2.9 that, if

$$
\mu_{n} \rightarrow \mu \text { weakly in } \mathfrak{M}(\partial \Omega), u_{n} \rightarrow u, \quad g\left(u_{n}\right) \rightarrow g(u) \text { in } L_{\rho}^{1}(\Omega),
$$

and if

$$
u_{n}=\mathbb{K}\left[\mu_{n}\right]-\mathbb{G}\left[g\left(u_{n}\right)\right],
$$

then $u=\mathbb{K}[\mu]-\mathbb{G}[g(u)]$.

Lemma 3.5. Let $\Omega$ be a Lipschitz bounded domain and let $g \in \mathcal{G}$. Suppose that $\mu \in \mathfrak{M}(\partial \Omega)$ and that there exists a solution of problem (3.7). Then the solution is unique.

If $\mu, \mu^{\prime}$ are two measures in $\mathfrak{M}(\partial \Omega)$, for which problem (3.7) possesses solutions $u, u^{\prime}$ respectively, then the following estimate holds:

$$
\begin{aligned}
\left\|u-u^{\prime}\right\|_{L_{\rho}^{1}(\Omega)}+\left\|g(u)-g\left(u^{\prime}\right)\right\|_{L_{\rho}^{1}(\Omega)} & \left.\leq\left\|\mathbb{K}\left[\mu-\mu^{\prime}\right]\right\|_{L_{\rho}^{1}(\Omega)}\right) \\
& \leq\left\|\mu-\mu^{\prime}\right\|_{\mathfrak{M}(\partial \Omega)} .
\end{aligned}
$$

If $\mu \leq \mu^{\prime}$ then $u \leq u^{\prime}$.

In addition, for any nonnegative element $\eta \in X(\Omega)$, we have

$$
-\int_{\Omega}(|u| \Delta \eta-|g(u)| \eta) d x \leq-\int_{\Omega} \mathbb{K}[|\mu|] \Delta \eta d x
$$

and

$$
-\int_{\Omega}\left(u_{+} \Delta \eta-g(u)_{+} \eta\right) d x \leq-\int_{\Omega} \mathbb{K}\left[\mu_{+}\right] \Delta \eta d x .
$$

Proof. This follows from Lemma 3.2 in the same way that Lemma 2.9 follows from Lemma 2.7.

Definition 3.6. Assume that $u \in W_{\text {loc }}^{1, p}(\Omega)$ for some $p>1$. We say that $u$ possesses a boundary trace $\mu \in \mathfrak{M}(\partial \Omega)$ if, for every Lipschitz exhaustion $\left\{\Omega_{n}\right\}$ of $\Omega$,

$$
\lim _{n \rightarrow \infty} \int_{\partial \Omega_{n}} Z u d \omega_{n}=\int_{\partial \Omega} Z d \mu
$$

holds for every $Z \in C(\bar{\Omega})$.

Similarly we say that $u$ possesses a trace $\mu$ on a relatively open set $A \subset \partial \Omega$ if (3.14) holds for every $Z \in C(\bar{\Omega})$ such that supp $Z \subset \Omega \cup A$. 
Remark 3.7. If $u \in W_{\text {loc }}^{1, p}(\Omega)$ for some $p>1$ then, by Sobolev's trace theorem, for every relatively open $(N-1)$ - dimensional Lipschitz surface $\Sigma, u$ possesses a trace in $W^{1-\frac{1}{p}, p}(\Sigma)$. In particular the trace is in $L^{1}(\Sigma)$. In fact there exists an element of the Lebesgue equivalence class of $u$ such that the trace on $\Sigma$ is precisely the restriction of $u$ to $\Sigma$. When it is relevant, as in (3.14), we assume that $u$ is represented by such an element.

If $u \in W^{1, p}(\Omega)$ then, by the same token, $u$ possesses a trace in $W^{1-\frac{1}{p}, p}(\partial \Omega)$. If $\left\{\Omega_{n}\right\}$ is a uniform Lipschitz exhaustion and $h_{n}$ (respectively $h$ ) denotes the trace of $u$ on $\partial \Omega_{n}$ (respectively $\partial \Omega$ ) then

$$
\left\|h_{n}\right\|_{W^{1-\frac{1}{p}, p}\left(\partial \Omega_{n}\right)} \rightarrow\|h\|_{W^{1-\frac{1}{p}, p}(\partial \Omega)} .
$$

This follows from the continuity of the imbedding

$$
W^{1, p}(\Omega) \hookrightarrow W^{1-\frac{1}{p}, p}(\partial \Omega)
$$

and the fact that $C^{1}(\bar{\Omega})$ is dense in $W^{1, p}(\Omega)$.

Similarly, if $\left\{\Omega_{n}\right\}$ is a Lipschitz exhaustion (not necessarily uniform, but satisfies (2.26)) then

$$
\left\|h_{n}\right\|_{L^{1}\left(\partial \Omega_{n}\right)} \rightarrow\|h\|_{L^{1}(\partial \Omega)} .
$$

In particular, if $u \in W_{0}^{1, p}(\Omega)$ then its boundary trace is zero, in the sense of the above definition.

Proposition 3.8. Let $u$ be a weak solution of (3.7). If $\left\{\Omega_{n}\right\}$ is a Lipschitz exhaustion of $\Omega$ then, for every $Z \in C(\bar{\Omega})$,

$$
\lim _{n \rightarrow \infty} \int_{\partial \Omega_{n}} Z u d \omega_{n}=\int_{\partial \Omega} Z d \mu
$$

where $\omega_{n}$ is the harmonic measure of $\Omega_{n}$ (relative to a point $x_{0} \in \Omega_{1}$ ).

Proof. If $v:=\mathbb{G}[g \circ u]$ then $v \in L_{\rho}^{1}(\Omega)$ and $u+v$ is a harmonic function. By (3.8), $u+v=\mathbb{K}^{\Omega}[\mu]$. Therefore, by Lemma 2.2,

$$
\lim _{n \rightarrow \infty} \int_{\partial \Omega_{n}} Z(u+v) d \omega_{n}=\int_{\partial \Omega} Z d \mu
$$

for every $Z \in C(\bar{\Omega})$. As $v \in W_{0}^{1, p}(\Omega)$ for some $p>1$ its boundary trace is zero. Therefore (3.16) implies (3.15).

Definition 3.9. A measure $\mu \in \mathfrak{M}(\partial \Omega)$ is called $g$-admissible if $g(\mathbb{K}[|\mu|]) \in$ $L_{\rho}^{1}(\Omega)$.

Theorem 3.10. If $\mu$ is g-admissible then problem (3.7) possesses a unique solution. 
Proof. First assume that $\mu>0$. Under the admissibility assumption, $U=\mathbb{K}[\mu]$ is a supersolution of (3.7). Let $\left\{D_{n}\right\}$ be an increasing sequence of smooth domains such that $\bar{D}_{n} \subset D_{n+1} \subset \Omega$ and $D_{n} \uparrow \Omega$. Let $u_{n}$ be the solution of problem (3.7) in $D_{n}$ with boundary data $h_{n}=\left.U\right|_{\partial D_{n}}$. Then $\left\{u_{n}\right\}$ decreases and the limit $u=\lim u_{n}$ satisfies (3.7).

In the general case we define $\bar{U}=\mathbb{K}[|\mu|]$ and $U, u_{n}$ as before. By assumption $g(\bar{U}) \in L_{\rho}^{1}(\Omega)$ and $\bar{U}$ dominates $\left|u_{n}\right|$ for all $n$. Let $\eta$ be a non-negative function in $X(\Omega)$ and let $\zeta_{n}$ be the solution of the problem

$$
\Delta \zeta=(\Delta \eta) \rho_{n} / \rho \text { in } D_{n}, \zeta=0 \text { on } \partial D_{n}
$$

Then $\zeta_{n} \in X\left(D_{n}\right)$ and, since $\rho_{n} \rightarrow \rho$,

$$
\left(\Delta \zeta_{n}\right) \rightarrow(\Delta \eta), \quad \zeta_{n} \rightarrow \eta
$$

In addition, $\left(\Delta \zeta_{n}\right) / \rho_{n}=(\Delta \eta) / \rho$ is bounded and, by (2.39), the sequence $\left\{\zeta_{n} / \rho_{n}\right\}$ is uniformly bounded.

The solutions $u_{n}$ satisfy,

$$
\int_{D_{n}}\left(-u_{n} \Delta \zeta_{n}+g\left(u_{n}\right) \zeta_{n}\right) d x=-\int_{D_{n}} \mathbb{P}^{D_{n}}\left[h_{n}\right] \Delta \zeta_{n} d x
$$

The sequence $\left\{u_{k}: k>n\right\}$ is bounded in $W^{1, p}\left(D_{n}\right)$ for every $n$. Consequently there exists a subsequence (still denoted by $\left\{u_{n}\right\}$ ) which converges pointwise a.e. in $\Omega$. We denote its limit by $u$. Since $\left\{u_{n}\right\}$ is dominated by $\bar{U}$ it follows that

$$
\lim _{n \rightarrow \infty} \int_{D_{n}}\left(-u_{n} \Delta \zeta_{n}+g\left(u_{n}\right) \zeta_{n}\right) d x=\int_{\Omega}(-u \Delta \eta+g(u) \eta) d x
$$

Furthermore,

$$
\int_{D_{n}} \mathbb{P}^{D_{n}}\left[h_{n}\right] \Delta \zeta_{n} d x=\int_{D_{n}} U \Delta \eta\left(\rho_{n} / \rho\right) d x \rightarrow \int_{\Omega} U \Delta \eta d x=\int_{\Omega} \mathbb{K}[\mu] \Delta \eta d x .
$$

Thus $u$ is the solution of (3.7).

Remark 3.11. If we do not assume that $g(0)=0$ the admissibility condition becomes,

$$
g\left(\mathbb{K}\left[\mu_{+}\right]+\rho(g(0))_{+}\right) \in L_{\rho}^{1}(\Omega) \quad \text { and } g\left(-\mathbb{K}\left[\mu_{-}\right]-\rho(g(0))_{-}\right) \in L_{\rho}^{1}(\Omega) .
$$

\section{The boundary trace of positive solutions}

As before we assume that $\Omega$ is a bounded Lipschitz domain and $g \in \mathcal{G}$. We denote by $\rho$ the first eigenfunction of $-\Delta$ in $\Omega$ normalized by $\rho\left(x_{0}\right)=1$ at some (fixed) point $x_{0} \in \Omega$. 
A function $u \in L_{\text {loc }}^{1}(\Omega)$ is a solution of the equation

$$
-\Delta u+g(u)=0 \quad \text { in } \Omega,
$$

if $g \circ u \in L_{\text {loc }}^{1}(\Omega)$ and $u$ satisfies the equation in the distribution sense.

A function $u \in L_{\text {loc }}^{1}(\Omega)$ is a supersolution (respectively subsolution) of the equation (4.1) if $g \circ u \in L_{\mathrm{loc}}^{1}(\Omega)$ and

$$
-\Delta u+g \circ u \geq 0 \quad(\text { respectively } \leq 0)
$$

in the distribution sense.

Proposition 4.1. Let $u$ be a positive solution of (4.1). If $g \circ u \in L_{\rho}^{1}(\Omega)$ then $u \in L_{\rho}^{1}(\Omega)$ and it possesses a boundary trace $\mu \in \mathfrak{M}(\partial \Omega)$, i.e., $u$ is the solution of the boundary value problem (3.7) with this measure $\mu$.

Proof. If $v:=\mathbb{G}[g \circ u]$ then $v \in L_{\rho}^{1}(\Omega)$ and $u+v$ is a positive harmonic function. Hence $u+v \in L_{\rho}^{1}(\Omega)$ and there exists a non-negative measure $\mu \in \mathfrak{M}(\partial \Omega)$ such that $u+v=\mathbb{K}[\mu]$. In view of (3.8), this implies our assertion.

Lemma 4.2. If $u$ is a non-negative solution of (4.1) then $u \in C^{1}(\Omega)$.

Let $\left\{u_{n}\right\}$ be a sequence of non-negative solutions of (4.1) which is uniformly bounded in every compact subset of $\Omega$. Then there exists a subsequence $\left\{u_{n_{j}}\right\}$ which converges in $C^{1}\left(\bar{\Omega}^{\prime}\right)$ for every $\Omega^{\prime} \Subset \Omega$ to a solution $u$ of $(4.1)$.

Proof. Since $g \circ u \in L_{\text {loc }}^{1}(\Omega)$ it follows that $u \in W_{\text {loc }}^{1, p}(\Omega)$ for some $p \in[1, N /(N-$ $1)$ ). Let $\Omega^{\prime}$ be a smooth domain such that $\Omega^{\prime} \Subset \Omega$. By the trace imbedding theorem, $u$ possesses a trace $h \in L^{1}\left(\partial \Omega^{\prime}\right)$. If $U$ is the harmonic function in $\Omega^{\prime}$ with boundary trace $h$ then $u<U$. Thus $u$ (and hence $g \circ u$ ) is bounded in every compact subset of $\Omega$. By elliptic p.d.e. estimates, $u \in C^{1}(\Omega)$.

The second assertion of the lemma follows from the first by a standard argument.

\section{Theorem 4.3.}

(i) Let $u$ be a non-negative supersolution (respectively subsolution) of (4.1). Then $u \in W_{\text {loc }}^{1, p}(\Omega)$ for some $p \in[1, N /(N-1))$. In particular, if $\Omega^{\prime}$ is a $C^{1}$ domain such that $\Omega^{\prime} \Subset \Omega$ then $u$ possesses a trace $h \in L^{1}\left(\partial \Omega^{\prime}\right)$.

(ii) If $u$ is a positive supersolution, there exists a non-negative solution $\underline{u} \leq u$ which is the largest among all solutions dominated by $u$.

If $u$ is a positive subsolution and $u$ is dominated by a solution $w$ of (4.1) then there exists a minimal solution $\bar{u}$ such that $u \leq \bar{u}$. In particular, if $g \in \mathcal{G}$ satisfies the Keller-Osserman condition then such a solution exists.

(iii) Under the assumptions of (ii), if $g \circ \underline{u} \in L_{\rho}^{1}(\Omega)$ (respectively $g \circ \bar{u} \in L_{\rho}^{1}(\Omega)$ ) then the boundary trace of $\underline{u}($ respectively $\bar{u})$ is also the boundary trace of $u$ in the sense of Definition 3.6. 
Proof. First consider the case of a supersolution. Since $-\Delta u+g(u) \geq 0$ there exists a positive Radon measure $\tau$ in $\Omega$ such that

$$
-\Delta u+g(u)=\tau \text { in } \Omega .
$$

Therefore $u \in W_{\text {loc }}^{1, p}(\Omega)$ and consequently $u$ possesses an $L^{1}$ trace on $\partial \Omega^{\prime}$ for every $\Omega^{\prime}$ as above.

Next, let $\left\{\Omega_{n}\right\}$ be a $C^{1}$ exhaustion of $\Omega$ which is also uniformly Lipschitz. Let $v_{n}$ be the solution of the boundary value problem

$$
-\Delta v+g(v)=0 \text { in } \Omega_{n}, v=u \text { on } \partial \Omega_{n} .
$$

Since $u$ possesses a trace in $L^{1}\left(\partial \Omega_{n}\right)$ this boundary value problem possesses a (unique) solution. By the comparison principle $0 \leq v_{n} \leq u$ in $\Omega_{n}$. Therefore the sequence $\left\{v_{n}\right\}$ decreases and consequently it converges to a solution $\underline{u}$ of (4.1). Evidently this is the largest solution dominated by $u$.

Now suppose that $g \circ \underline{u} \in L_{\rho}^{1}(\Omega)$ (but not necessarily $g \circ u \in L_{\rho}^{1}(\Omega)$ ). By Proposition 4.1, $\underline{u} \in L_{\rho}^{1}(\Omega)$ and $\underline{u}$ possesses a boundary trace $\mu$. By the definition of $v_{n}$,

$$
\begin{aligned}
\int_{\partial \Omega_{n}} u d \omega_{n}=\int_{\partial \Omega_{n}} P^{\Omega_{n}}\left(x_{0}, y\right) u(y) d S & =v_{n}\left(x_{0}\right)+\int_{\Omega_{n}} G^{\Omega_{n}}\left(x, x_{0}\right) g\left(v_{n}(x)\right) d x \\
& \rightarrow \underline{u}\left(x_{0}\right)+\int_{\Omega} G^{\Omega}\left(x, x_{0}\right) g(\underline{u}(x)) d x .
\end{aligned}
$$

Hence, taking a subsequence if necessary, we may assume that

$$
u \chi_{\partial \Omega_{n}} \omega_{n} \rightarrow \mu^{\prime}
$$

where $\mu^{\prime}$ is a measure on $\partial \Omega$ such that

$$
\mu^{\prime}(\partial \Omega)=\underline{u}\left(x_{0}\right)+\int_{\Omega} G^{\Omega}\left(x, x_{0}\right) g(\underline{u}(x)) d x .
$$

On the other hand, as $\mu$ is the boundary trace of $\underline{u}$,

$$
\underline{u}\left(x_{0}\right)+\int_{\Omega} G^{\Omega}\left(x, x_{0}\right) g(\underline{u}(x)) d x=\mu(\partial \Omega) .
$$

Thus $\mu(\partial \Omega)=\mu^{\prime}(\partial \Omega)$. However, as $\underline{u} \leq u$, we have $\mu \leq \mu^{\prime}$. This implies that $\mu=\mu^{\prime}$.

Next we treat the case of a subsolution. The proof of (i) is the same as before. We turn to (ii). In the present case, the corresponding sequence $\left\{v_{n}\right\}$ is increasing and, in general, may not converge. But, as we assume that $u$ is dominated by a solution $w$, the sequence converges to a solution $\bar{u}$ which is clearly the smallest solution above $u$. In particular, if $g$ satisfies the Keller-Osserman condition then $\left\{v_{n}\right\}$ is uniformly bounded in every compact subset of $\Omega$ and consequently converges to a solution.

The proof of (iii) for subsolutions is again the same as in the case of supersolutions. 
Corollary 4.4. I. Let u be a non-negative supersolution of (4.1). Let A be a relatively open subset of $\partial \Omega$. Suppose that, for every Lipschitz domain $\Omega^{\prime}$ such that

$$
\Omega^{\prime} \subset \Omega, \quad \partial \Omega^{\prime} \cap \partial \Omega \subset A,
$$

we have

$$
g \circ u \in L_{\rho}^{1}\left(\Omega^{\prime}\right) .
$$

Then both $u$ and $\underline{u}$ possess traces on $A$ and the two traces are equal.

II. Let $u$ be a non-negative subsolution of (4.1). Let A be a relatively open subset of $\partial \Omega$. Suppose that for every Lipschitz domain $\Omega^{\prime}$ satisfying (4.3) we have

$$
g \circ \bar{u} \in L_{\rho}^{1}\left(\Omega^{\prime}\right) .
$$

Then both $u$ and $\bar{u}$ posses traces on $A$ and the two traces are equal.

Proof. Let $u$ be a supersolution and let $\Omega^{\prime}$ be a domain as above. Denote by $\rho^{\prime}$ the first eigenfunction of $-\Delta$ in $\Omega^{\prime}$ normalized by $\rho^{\prime}\left(x_{0}\right)=1$ for some $x_{0} \in \Omega^{\prime}$. Since $\rho^{\prime} \leq c \rho$, (4.3) implies that $g \circ u \in L_{\rho^{\prime}}^{1}\left(\Omega^{\prime}\right)$. Let $\underline{u}^{\prime}$ denote the largest solution of (4.1) in $\Omega^{\prime}$ dominated by $u$. Then $g \circ \underline{u}^{\prime} \in L_{\rho^{\prime}}^{1}\left(\Omega^{\prime}\right)$ and, by Theorem 4.3, $\underline{u}^{\prime} \in L_{\rho}^{1}\left(\Omega^{\prime}\right)$ and $\underline{u}^{\prime}$ has a trace $v^{\prime}$ on $\partial \Omega^{\prime}$ which is also the boundary trace of $u$ on $\partial \Omega$.

Let $\left\{\Omega_{n}\right\}$ be an increasing uniformly Lipschitz sequence of domains such that $\partial \Omega_{n} \cap \Omega$ is a $C^{1}$ surface, $D_{n}:=\Omega \backslash \Omega_{n}$ is Lipschitz and

$$
F_{n}:=\partial \Omega_{n} \backslash \Omega \subset F_{n+1}^{0} \subset A, \quad \cup \Omega_{n}=\Omega, \quad \cup F_{n}^{0}=A,
$$

where $F_{n}^{0}$ is the relative interior of $F_{n}$. Denote by $\underline{u}_{n}$ the largest solution dominated by $u$ in $\Omega_{n}$ and observe that $\left\{\underline{u}_{n}\right\}$ is decreasing and converges to a solution. Obviously this is the largest solution dominated by $u$, namely, $\underline{u}$.

Let $\tau_{n}$ be the trace of $\underline{u}_{n}$ on $\partial \Omega_{n}$. Put $v_{n}=\tau_{n} \chi_{F_{n}}$. Recall that $\tau_{n}$ is also the trace of $u$ so that

$$
v_{n}^{\prime}=\tau_{n}-v_{n}=u \chi_{\partial \Omega_{n} \backslash F_{n}} d S .
$$

Assertion A. There exists a Radon measure $v$ on $A$ such that $v_{n} \rightarrow v$ and $v$ is the trace of $u$, as well as of $\underline{u}$, on $A$.

Let $E$ be a compact subset of $A$ and denote,

$$
n(E):=\inf \left\{m \in \mathbb{N}: E \subset F_{m}^{0}\right\} .
$$

In view of the fact that, for $n \geq n(E), v_{n}$ is the trace of $u$, relative to $\Omega_{n}$, on a set $F_{n(E)}^{0}$ in which $E$ is strongly contained and the fact that $\left\{\Omega_{n}\right\}$ is Lipschitz, Lemma 2.5 implies that the set $\left\{v_{n}(E): n \geq n(E)\right\}$ is bounded. By taking a sequence if necessary we may assume that

$$
v_{n} L_{E} \rightarrow v_{E} .
$$


Applying this procedure to $E=F_{m}$ for each $m \in \mathbb{N}$ and then using the diagonalization method we obtain a subsequence, again denoted by $\left\{v_{n}\right\}$, such that

$$
v_{n} \rightarrow v
$$

where $v$ is a Radon measure on $A$ (not necessarily bounded).

Next we wish to show that $v$ is the trace of $u$ on $A$ relative to $\Omega$. To this purpose we construct a $C^{1}$ exhaustion of $\Omega$, say $\left\{D_{n}\right\}$, such that $D_{n} \Subset \Omega_{n}$ and ว $D_{n}=\Gamma_{n} \cup \Gamma_{n}^{\prime}$ where

$$
\begin{aligned}
& \Gamma_{n}^{\prime}=\partial \Omega_{n} \cap\left\{y \in \Omega: \operatorname{dist}\left(y, F_{n}\right) \geq \epsilon_{n}\right\} \\
& \Gamma_{n} \subset\left\{y \in \Omega_{n}: \operatorname{dist}\left(y, F_{n}\right)<\epsilon_{n}\right\},
\end{aligned}
$$

where $0<\epsilon_{n}<\frac{1}{2} \operatorname{dist}\left(F_{n}, \partial \Omega \backslash A\right)$ is chosen so that

$$
\mathbb{H}_{N-1} \chi_{\Gamma_{n}} \rightarrow \mathbb{H}_{N-1} \chi_{A} \text { and } u \chi_{\Gamma_{n}} d \omega^{n} \rightarrow v .
$$

Here $d \omega^{n}$ is the harmonic measure in $D_{n}$. This is possible because, if $\Gamma_{n}$ is sufficiently close to $\partial \Omega_{n}$, then

$$
u \chi \Gamma_{n} d \omega^{n}-v_{n} \chi F_{n} \rightarrow 0 .
$$

(As usual in this paper, $v_{n} \chi_{F_{n}}$ denotes the Borel measure in $\mathbb{R}^{N}$ that is equal to $v_{n}$ on $F_{n}$ and zero elsewhere.) This implies that $v$ is the trace of $u$ on $A$. to $\partial \Omega_{n}$,

Since $v_{n}$ is also the trace of $\underline{u}_{n}$ on $F_{n}$ it follows that, if $\Gamma_{n}$ is sufficiently close

$$
\underline{u}_{n} \chi_{\Gamma_{n}} d \omega^{n}-v_{n} \chi_{F_{n}} \rightarrow 0 .
$$

As $\underline{u}_{n} \downarrow \underline{u}$ we deduce that $v$ is also the trace of $\underline{u}$ on $A$.

If $u$ is a subsolution the argument is essentially the same. Let $\bar{u}_{n}$ be the smallest solution that dominates $u$ in $\Omega_{n}$. Then the sequence $\left\{\bar{u}_{n}\right\}$ is increasing, but it is dominated by a solution $w$. Therefore it converges to a solution and this is the smallest solution dominating $u$, namely, $\bar{u}$. By Theorem $4.3, \underline{u}_{n}$ and $u\lfloor$ possess the same trace on $\partial \Omega_{n}$. Let $\tau_{n}$ be the trace of $\underline{u}_{n}$ on $\partial \Omega_{n}$ and put $v_{n}=\tau_{n} \chi_{F_{n}}$. The rest of the proof is as before.

Definition 4.5. Let $u$ be a positive supersolution, respectively subsolution, of (4.1). A point $y \in \partial \Omega$ is a regular boundary point relative to $u$ if there exists an open neighborhood $D$ of $y$ such that $g \circ u \in L_{\rho}^{1}(\Omega \cap D)$. If no such neighborhood exists we say that $y$ is a singular boundary point relative to $u$.

The set of regular boundary points of $u$ is denoted by $\mathcal{R}(u)$; its complement on the boundary is denoted by $\mathcal{S}(u)$. Evidently $\mathcal{R}(u)$ is relatively open.

Theorem 4.6. Let $u$ be a positive solution of (4.1) in $\Omega$. Then u possesses a trace on $\mathcal{R}(u)$, given by a Radon measure $v$. 
Furthermore, for every compact set $F \subset \mathcal{R}(u)$,

$$
\int_{\Omega}(-u \Delta \eta+g(u) \eta) d x=-\int_{\Omega}\left(\mathbb{K}\left[v \chi_{F}\right] \Delta \eta\right) d x
$$

for every $\eta \in X(\Omega)$ such that supp $\eta \cap \partial \Omega \subset F$.

Proof. The first assertion is an immediate consequence of Corollary 4.4.

We turn to the proof of the second assertion. Let $F$ be a compact subset of $\mathcal{R}(u)$ and let $\eta \in X(\Omega)$ be a function such that the following conditions hold for some open set $E_{\eta}$ :

$$
\operatorname{supp} \eta \subset \bar{\Omega} \cap E_{\eta}, \quad F \subset E_{\eta} \cap \partial \Omega, \quad \bar{E}_{\eta} \cap \mathcal{S}(u)=\emptyset, \quad x_{0} \in D_{\eta}:=\Omega \cap E_{\eta} .
$$

By Definition 4.5, if $D$ is a subdomain of $\Omega$ such that $\bar{D} \cap \mathcal{S}(u)=\emptyset$ then $g \circ u \in$ $L_{\rho}^{1}(D)$, where $\rho$ is the first normalized eigenfunction of $\Omega$. Let $E$ be a $C^{2}$ domain such that

$$
\bar{E}_{\eta} \subset E, \quad \mathbb{H}_{N-1}(\partial \Omega \cap \partial E)=0, \quad \bar{E} \cap \mathcal{S}(u)=\emptyset .
$$

Put $D:=E \cap \Omega$ and note that $g \circ u \in L_{\rho}^{1}(D)$.

If $\phi$ denotes the first normalized eigenfunction in $D$ then $\phi \leq c \rho$ for some positive constant $c$. Therefore the fact that $g \circ u \in L_{\rho}^{1}(D)$ implies that $g \circ u \in L_{\phi}^{1}(D)$ and the properties of $\eta$ imply that $\eta \in X(D)$. Hence $u$ possesses a boundary trace $\tau^{D}$ on $\partial D$ and

$$
\int_{D}(-u \Delta \eta+g(u) \eta) d x=-\int_{D}\left(\mathbb{K}^{D}\left[\tau^{D}\right] \Delta \eta\right) d x .
$$

Let $\Gamma=\bar{E} \cap \partial \Omega$ and $\Gamma^{\prime}=\partial D \backslash \Gamma$; note that $\Gamma \cap \mathcal{S}(u)=\emptyset$ and $\eta$ vanishes in a neighborhood of $\partial E \cap \bar{\Omega}$. Put $\tau_{\Gamma}^{D}=\tau^{D} \chi_{\Gamma}$ and $\tau_{\Gamma^{\prime}}^{D}=\tau^{D}-\tau_{\Gamma}^{D}$. Then $d \tau_{\Gamma^{\prime}}^{D}=u d S$ on $\Gamma^{\prime}$ and, as $u \in C(\bar{D} \backslash \Gamma)$,

$$
\mathbb{K}^{D}\left[\tau_{\Gamma^{\prime}}^{D}\right] \in C(\bar{D} \backslash \Gamma) .
$$

Furthermore $\eta$ vanishes in a neighborhood of $\Gamma^{\prime}$ and consequently

$$
\begin{aligned}
\int_{D}\left(\mathbb{K}^{D}\left[\tau_{\Gamma^{\prime}}^{D}\right] \Delta \eta\right) d x & =\int_{D}\left(\int_{\partial D \backslash \Gamma} P^{D}(x, y) u(y) d S_{y}\right) \Delta \eta(x) d x \\
& =\int_{\partial D \backslash \Gamma}\left(\int_{D} P^{D}(x, y) \Delta \eta(x) d x\right) u(y) d S_{y}=0 .
\end{aligned}
$$

Thus

$$
\int_{\Omega}(-u \Delta \eta+g(u) \eta) d x=-\int_{\Omega} \mathbb{K}^{D}\left[\tau_{\Gamma}^{D}\right] \Delta \eta d x .
$$

(Changing the domain of integration from $D$ to $\Omega$ makes no difference since $\eta$ vanishes in $\Omega \backslash D$.) 
Now, $\tau_{\Gamma}^{D}$ is the trace of $u$ on $\Gamma$ relative to $D$ while $v \chi_{\Gamma}$ is the trace of $u$ on $\Gamma$ relative to $\Omega$. Since $D \subset \Omega$ it follows that

$$
\tau_{\Gamma}^{D} \leq v \chi_{\Gamma}
$$

Let $\left\{E^{j}\right\}$ be an increasing sequence of $C^{2}$ domains such that each domain possesses the same properties as $E$ and,

$$
\bar{E}^{j} \cap \partial \Omega=\bar{E} \cap \partial \Omega=\Gamma, \text { and } D^{j}:=E^{j} \cap \Omega \uparrow \Omega \text {. }
$$

For each $j \in \mathbb{N}$ and $y \in \Gamma$, the function $K^{D^{j}}(\cdot, y)$ is harmonic in $D^{j}$, vanishes on $\partial D^{j} \backslash\{y\}$ and $K^{D^{j}}\left(x_{0}, y\right)=1$. Furthermore the sequence $\left\{K^{D^{j}}(\cdot, y)\right\}$ is nondecreasing. Therefore it converges uniformly in compact subsets of $(\Omega \cup \Gamma) \backslash\{y\}$. The limit is the corresponding kernel function in $\Omega$, namely $K^{\Omega}(\cdot, y)$. (Recall that the kernel function is unique.)

In view of (4.9), the sequence $\left\{\tau_{\Gamma}^{D^{j}}\right\}$ is bounded. Therefore there exists a subsequence, which we still denote by $\left\{\tau_{\Gamma}^{D^{j}}\right\}$, such that

$$
\tau_{\Gamma}^{D^{j}} \rightarrow \tau_{\Gamma}
$$

weakly relative to $C(\Gamma)$. Combining these facts we obtain,

$$
\mathbb{K}^{D_{j}}\left[\tau_{\Gamma}^{D^{j}}\right] \rightarrow \mathbb{K}^{\Omega}\left[\tau_{\Gamma}\right]
$$

Hence, by (4.7),

$$
\int_{\Omega}(-u \Delta \eta+g(u) \eta) d x=-\int_{\Omega}\left(\mathbb{K}^{\Omega}\left[\tau_{\Gamma}\right] \Delta \eta\right) d x .
$$

Finally, as $\tau_{\Gamma}^{D_{j}}$ is the trace of $u$ on $\Gamma$ relative to $D_{j}$ then, in view of (4.10), the limit $\tau_{\Gamma}$ is the trace of $u$ on $\Gamma$ relative to $\Omega$, i.e.,

$$
\tau_{\Gamma}=v \chi_{\Gamma}
$$

This relation and (4.11) imply (4.6).

Theorem 4.7. I. Let $u$ be a positive supersolution of (4.1) in $\Omega$ and let $\underline{u}$ be the largest solution dominated by $u$. Then,

$$
\mathcal{S}(u)=\mathcal{S}(\underline{u}), \quad \mathcal{R}(u)=\mathcal{R}(\underline{u}) .
$$

Both $u$ and $\underline{u}$ possess a trace on $\mathcal{R}(u)$ and the two traces are equal.

II. Let $u$ be a positive subsolution of (4.1) in $\Omega$ and let $\bar{u}$ be the smallest solution which dominates $u$. If $u$ is dominated by a solution $w$ of (4.1) then both $u$ and $\bar{u}$ 
possess a trace on $\mathcal{R}(w)$ (which is contained in $\mathcal{R}(u)$ ) and the two traces are equal on this set.

In particular, if $\mathcal{R}(w)=\mathcal{R}(u)$ then (4.12), with $\underline{u}$ replaced by $\bar{u}$, holds and both $u$ and $\bar{u}$ possess a trace on $\mathcal{R}(u)$, the two traces being equal.

III. Let $v$ denote the trace of $u$ on $\mathcal{R}(u)$. Then, for every compact set $F \subset \mathcal{R}(u)$,

$$
\int_{\Omega}(-u \Delta \eta+g(u) \eta) d x \begin{cases}\geq-\int_{\Omega}\left(\mathbb{K}\left[v \chi_{F}\right] \Delta \eta\right) d x, & \text { u supersolution, } \\ \leq-\int_{\Omega}\left(\mathbb{K}\left[v \chi_{F}\right] \Delta \eta\right) d x, & \text { u subsolution }\end{cases}
$$

for every $\eta \in X(\Omega), \eta \geq 0$, such that $\operatorname{supp} \eta \cap \partial \Omega \subset F$.

Proof. Part I. is a consequence of Corollary $4.4 \mathrm{I}$.

The first assertion in II. follows from Corollary 4.4 II. with $A=\mathcal{R}(w)$. The second assertion in II. is an immediate consequence of the first.

By Theorem 4.6, $\underline{u}$ (respectively $\bar{u}$ ) satisfy (4.6), where $v$ is the trace of $\underline{u}$ (respectively $\bar{u}$ ) on $\mathcal{R}(u)$. Since $v$ is also the trace of $u$ on $\mathcal{R}(u)$ we obtain statement III.

Theorem 4.8. Assume that $g \in \mathcal{G}$ satisfies the Keller-Osserman condition.

(i) Let $u$ be a positive solution of (4.1) and let $\left\{\Omega_{n}\right\}$ be a Lipschitz exhaustion of $\Omega$. If $y \in \mathcal{S}(u)$ then, for every nonnegative $Z \in C(\bar{\Omega})$ such that $Z(y) \neq 0$

$$
\lim \int_{\partial \Omega_{n}} Z u d \omega_{n}=\infty .
$$

(ii) Let $u$ be a positive supersolution of (4.1) and let $\left\{\Omega_{n}\right\}$ be a $C^{1}$ exhaustion of $\Omega$. If $y \in \mathcal{S}(u)$ then (4.14) holds for every nonnegative $Z \in C(\bar{\Omega})$ such that $Z(y) \neq 0$.

Proof. The proof of satement (i) is essentially the same as for the corresponding result in smooth domains [23, Lemma 2.8] and therefore will be omitted. In fact the assumption that $g$ satisfies the Keller-Osserman condition implies that the set of conditions II in [23, Lemma 2.8] is satisfied. Here too, the Keller-Osserman condition can be replaced by the weaker set of conditions II in the same way as in [23].

Part (ii) is a consequence of Theorem 4.7 and statement (i).

Definition 4.9. Let $g \in \mathcal{G}$. Let $u$ be a positive solution of (4.1) with regular boundary set $\mathcal{R}(u)$ and singular boundary set $\mathcal{S}(u)$. The Radon measure $v$ in $\mathcal{R}(u)$ associated with $u$ as in Theorem 4.6 is called the regular part of the trace of $u$. The couple $(\nu, \mathcal{S}(u))$ is called the boundary trace of $u$ on $\partial \Omega$. This trace is also represented by the (possibly unbounded) Borel measure $\bar{v}$ given by

$$
\bar{v}(E)= \begin{cases}v(E), & \text { if } E \subset \mathcal{R}(u) \\ \infty, & \text { otherwise }\end{cases}
$$


The boundary trace of $u$ in the sense of this definition will be denoted by $\operatorname{tr}_{\partial \Omega} u$.

Let

$$
V_{v}:=\sup \left\{u_{\nu_{\chi_{F}}}: F \subset \mathcal{R}(u), F \text { compact }\right\}
$$

where $u_{v \chi_{F}}$ denotes the solution of (3.7) with $\mu=v \chi_{F}$. Then $V_{v}$ is called the semi-regular component of $u$.

Remark 4.10. Let $\tau$ be a Radon measure on a relatively open set $A \subset \partial \Omega$. Suppose that for every compact set $F \subset A, u_{\tau \chi_{F}}$ is defined. If $V_{\tau}$ is defined as above, it need not be a solution of (4.1) or even be finite. However, if $g$ satisfies the KellerOsserman condition or if $u_{\tau \chi_{F}}$ is dominated by a solution $w$, independent of $F$, then $V_{\tau}$ is a solution.

Definition 4.11. A compact set $F \subset \partial \Omega$ is removable relative to (4.1) if the only non-negative solution $u \in C(\bar{\Omega} \backslash F)$ which vanishes on $\bar{\Omega} \backslash F$ is the trivial solution $u=0$.

Remark 4.12. In the case of power nonlinearities in smooth domains there exists a complete characterization of removable sets (see [22] and the references therein).

Lemma 4.13. Let $g \in \mathcal{G}$ and assume that $g$ satisfies the Keller-Osserman condition. Let $F \subset \partial \Omega$ be a compact set and denote by $\mathcal{U}_{F}$ the class of solutions $u$ of (4.1) which satisfy the condition,

$$
u \in C(\bar{\Omega} \backslash F), \quad u=0 \text { on } \partial \Omega \backslash F .
$$

Then there exists a function $U_{F} \in \mathcal{U}_{F}$ such that

$$
u \leq U_{F} \quad \forall u \in \mathcal{U}_{F} .
$$

Furthermore, $\mathcal{S}\left(U_{F}\right)=: F^{\prime} \subset F ; F^{\prime}$ need not be equal to $F$.

The proof is standard and will be omitted.

Definition 4.14. $U_{F}$ is called the maximal solution associated with $F$. The set $F^{\prime}=\mathcal{S}\left(U_{F}\right)$ is called the $g$-kernel of $F$ and denoted by $k_{g}(F)$.

Note. The situation $\mathcal{S}\left(U_{F}\right) \subsetneq F$ occurs if and only if there exists a closed set $F^{\prime} \subset F$ such that $F \backslash F^{\prime}$ is a non-empty removable set. In this case $U_{F}=U_{F^{\prime}}$.

Lemma 4.15. Let $F_{1}, F_{2}$ be two compact subsets of $\partial \Omega$. Then,

$$
F_{1} \subset F_{2} \Longrightarrow U_{F_{1}} \leq U_{F_{2}}
$$

and

$$
U_{F_{1} \cup F_{2}} \leq U_{F_{1}}+U_{F_{2}} \text {. }
$$

If $F$ is a compact subset of $\partial \Omega$ and $\left\{N_{k}\right\}$ is a decreasing sequence of relatively open neighborhoods of $F$ such that $\bar{N}_{k+1} \subset N_{k}$ and $\cap N_{k}=F$ then

$$
U_{\bar{N}_{k}} \rightarrow U_{F}
$$

uniformly in compact subsets of $\Omega$. 
Proof. The first statement is an immediate consequence of the definition of maximal solution.

Next we verify (4.20). By (4.18) the sequence $\left\{U_{\bar{N}_{k}}\right\}$ decreases and therefore it converges to a solution $U$. Clearly $U$ has trace zero outside $F$ so that $U \leq U_{F}$ On the other hand, for every $k, U_{\bar{N}_{k}} \geq U_{F}$. Hence $U=U_{F}$.

We turn to the verification of (4.19). Let $u$ be a positive solution of (5.1) which vanishes on $\partial \Omega \backslash\left(F_{1} \cup F_{2}\right)$. We shall show that there exists solutions $u_{1}, u_{2}$ of (5.1) such that

$$
u_{i}=0 \text { on } \partial \Omega \backslash F_{i}, u \leq u_{1}+u_{2} .
$$

First we prove this statement in the case where $F_{1} \cap F_{2}=\emptyset$. Let $E_{1}, E_{2}$ be $C^{1}$ domains such that $\bar{E}_{1} \cap \bar{E}_{2}=\varnothing$ and $F_{i} \subset E_{i} \cap \partial \Omega$, (i=1,2). Let $\left\{\Omega_{n}\right\}$ be a Lipschitz exhaustion of $\Omega$ and put $A_{n, i}=\partial \Omega_{n} \cap E_{i}$, (i=1,2). Let $v_{n, i}$ be the solution of (5.1) in $\Omega_{n}$ with boundary data $u \chi_{A_{n, i}}$ and $v_{n}$ be the solution in $\Omega_{n}$ with boundary data $u\left(1-\chi_{A_{n, 1} \cup A_{n, 2}}\right)$. Then

$$
u \leq v_{n}+v_{n, 1}+v_{n, 2}
$$

By taking a subsequence if necessary we may assume that the sequences $\left\{v_{n}\right\}$, $\left\{v_{n, 1}\right\},\left\{v_{n, 2}\right\}$ converge. Then $\lim v_{n, i}=U_{i}$ where $U_{i}$ vanishes on $\partial \Omega \backslash E_{i},(\mathrm{i}=1,2)$. In addition, as the trace of $u$ on $\partial \Omega \backslash\left(F_{1} \cup F_{2}\right)$ is zero, we have $\lim v_{n}=0$. Thus

$$
u \leq U_{1}+U_{2}
$$

Now take decreasing sequences of $C^{1}$ domains $\left\{E_{k, 1}\right\},\left\{E_{k, 2}\right\}$ such that

$$
\bar{E}_{k, 1} \cap \bar{E}_{k, 2}=\emptyset, \quad F_{i} \subset E_{k, i} \cap \partial \Omega, \quad \bar{E}_{k, i} \cap \partial \Omega \downarrow F_{i} \quad i=1,2 .
$$

Construct $U_{k, i}$ corresponding to $E_{k, i}$ in the same way that $U_{i}$ corresponds to $E_{i}$. Then,

$$
u \leq U_{k, 1}+U_{k, 2}
$$

and, by (4.20), taking a subsequence if necessary,

$$
u_{i}:=\lim _{k \rightarrow \infty} U_{k, i}=0 \text { on } \partial \Omega \backslash F_{i}, \quad i=1,2
$$

This proves (4.21) in the case where $F_{1}, F_{2}$ are disjoint.

In the general case, let $\left\{N_{j}\right\}$ be a decreasing sequence of relatively open neighborhoods of $F_{1} \cap F_{2}$ such that

$$
\bar{N}_{j+1} \subset N_{j}, \quad \cap N_{j}=F_{1} \cap F_{2}
$$

Put $F_{j, 2}^{\prime}=F_{2} \backslash N_{j}$. 
Let $\left\{M_{j}\right\}$ be a decreasing sequence of relatively open neighborhoods of $F_{1}$ such that

$$
\bar{M}_{j+1} \subset M_{j}, \quad \cap M_{j}=F_{1}, \quad \bar{M}_{j} \cap F_{j, 2}^{\prime}=\emptyset .
$$

Put $F_{j, 1}^{\prime}:=\bar{M}_{j}$.

Let $v_{j}$ be the largest solution dominated by $u$ and vanishing on the complement of $F_{j, 1}^{\prime} \cup F_{j, 2}^{\prime}$ :

$$
\begin{aligned}
\partial \Omega \backslash\left(F_{j, 1}^{\prime} \cup F_{j, 2}^{\prime}\right) & =\partial \Omega \backslash\left(\left(F_{1} \cup F_{2}\right) \backslash\left(N_{j} \backslash \bar{M}_{j}\right)\right) \\
& =\left(\partial \Omega \backslash\left(F_{1} \cup F_{2}\right)\right) \cup\left(N_{j} \backslash \bar{M}_{j}\right) .
\end{aligned}
$$

Furthermore, $\left(u-U_{\bar{N}_{j} \backslash M_{j}}\right)_{+}$is a subsolution which is dominated by $u$ and vanishes on the complement of $F_{j, 1}^{\prime} \cup F_{j, 2}^{\prime}$. Therefore $v_{j}$ satisfies

$$
u \geq v_{j} \geq\left(u-U_{\bar{N}_{j} \backslash M_{j}}\right)_{+},
$$

which implies,

$$
0 \leq u-v_{j} \leq U_{\bar{N}_{j} \backslash M_{j}} \leq U_{\bar{N}_{j}} .
$$

By (4.20), $U_{\bar{N}_{j}} \downarrow U_{F_{1} \cap F_{2}}$. Taking a converging subsequence $v_{j_{i}} \rightarrow v$ we obtain

$$
0 \leq u-v \leq U_{F_{1} \cap F_{2}} .
$$

By the previous part of the proof there exist solutions $v_{j, 1}, v_{j, 2}$, whose boundary trace is supported in $F_{j, 1}^{\prime}$ and $F_{j, 2}^{\prime}$ respectively, such that

$$
v_{j} \leq v_{j, 1}+v_{j, 2} \text {. }
$$

Taking a subsequence we may assume convergence of $\left\{v_{j, 1}\right\}$ and $\left\{v_{j, 2}\right\}$. Then $u_{i}=$ $\lim v_{j, i}$ has boundary trace supported in $F_{i}$. Finally,

$$
u \leq v+U_{F_{1} \cap F_{2}} \leq u_{1}+u_{2}+U_{F_{1} \cap F_{2}}
$$

and $\operatorname{tr}_{\partial \Omega} u_{1}$ is supported in $F_{1}$ while $\operatorname{tr}_{\partial \Omega}\left(u_{2}+U_{F_{1} \cap F_{2}}\right)$ is supported in $F_{2}$. Since $u-u_{1}$ is a subsolution dominated by the supersolution $u_{2}+U_{F_{1} \cap F_{2}}$ there exists a solution $w_{2}$ between them and we obtain

$$
u \leq u_{1}+w_{2}
$$

where $\operatorname{tr}_{\partial \Omega} w_{2}$ is supported in $F_{2}$.

The next theorem deals with some aspects of the generalized boundary value problem:

$$
\begin{aligned}
-\Delta u+g \circ u & =0, \quad u \geq 0 \text { in } \Omega, \\
\operatorname{tr}_{\partial \Omega} & =(v, F),
\end{aligned}
$$

where $F \subset \partial \Omega$ is a compact set and $v$ is a (non-negative) Radon measure on $\partial \Omega \backslash F$. 
Theorem 4.16. Let $g \in \mathcal{G}$ and assume that $g$ is convex and satisfies the KellerOsserman condition.

EXISTENCE. The following set of conditions is necessary and sufficient for the existence of a solution $u$ of (4.22):

(i) For every compact set $E \subset \partial \Omega \backslash F$, the problem

$$
-\Delta u+g(u)=0 \text { in } \Omega, u=v \chi_{E} \text { on } \partial \Omega,
$$

possesses a solution.

(ii) If $k_{g}(F)=F^{\prime}$, then $F \backslash F^{\prime} \subset \mathcal{S}\left(V_{v}\right)$.

When this holds,

$$
V_{v} \leq u \leq V_{v}+U_{F} .
$$

Furthermore if $F$ is a removable set then (4.22) possesses exactly one solution.

UNIQUENESS. Given a compact set $F \subset \partial \Omega$, assume that

$$
U_{E} \text { is the unique solution with trace }\left(0, k_{g}(E)\right)
$$

for every compact $E \subset F$. Under this assumption:

(a) If $u$ is a solution of (4.22) then

$$
\max \left(V_{v}, U_{F}\right) \leq u \leq V_{v}+U_{F} .
$$

(b) Equation (5.1) possesses at most one solution satisfying (4.26).

(c) Condition (4.25) is necessary and sufficient in order that (4.22) posses at most one solution.

\section{MONOTONICITY.}

(d) Let $u_{1}, u_{2}$ be two positive solutions of (4.1) with boundary traces $\left(v_{1}, F_{1}\right)$ and $\left(\nu_{2}, F_{2}\right)$ respectively. Suppose that $F_{1} \subset F_{2}$ and that $v_{1} \leq v_{2} \chi_{F_{1}}=: v_{2}^{\prime}$. If (4.25) holds for $F=F_{2}$ then $u_{1} \leq u_{2}$.

Proof. First assume that there exists a solution $u$ of (4.22). By Theorem 4.6 condition (i) holds. Consequently $V_{v}$ is well defined by (4.16).

Since $V_{v} \leq u$ the function $w:=u-V_{v}$ is a subsolution of (4.1). Indeed, as $g$ is convex and $g(0)=0$ we have

$$
g(a)+g(b) \leq g(a+b) \quad \forall a, b \in \mathbb{R}_{+} .
$$

Therefore

$$
0=-\Delta w+\left(g(u)-g\left(V_{v}\right) \geq-\Delta w+g(w) .\right.
$$

By Theorem 4.3, as $g$ satisfies the Keller-Osserman condition, there exists a solution $\bar{w}$ of (4.1) which is the smallest solution dominating $w$. 
By Theorem 4.7, the traces of $w$ and $\bar{w}$ are equal on $A=\mathcal{R}(u) \subset \mathcal{R}(\bar{w})$. Clearly the trace of $w$ on $\mathcal{R}(u)$ is zero. The definitions of $V_{v}$ and $\bar{w}$ imply,

$$
\max \left(V_{v}, \bar{w}\right) \leq u \leq V_{v}+\bar{w} .
$$

Therefore

$$
\mathcal{S}(\bar{w}) \cup \mathcal{S}\left(V_{v}\right)=\mathcal{S}(u) .
$$

In addition, as $\bar{w}$ has trace zero in $\partial \Omega \backslash F$, it follows, by the definition of the maximal function, that

$$
\bar{w} \leq U_{F} \text { and consequently } \mathcal{S}(\bar{w}) \subset k_{g}(F) .
$$

These observations imply that condition (ii) must hold. Inequality (4.24) follows from (4.28) and this inequality implies that if $F$ is a removable set then (4.22) possesses exactly one solution.

Now we assume that conditions (i) and (ii) hold and prove existence of a solution. The function $V_{v}$ is well defined and $V_{v}+U_{F}$ is a supersolution of (4.1) whose boundary trace is $(\nu, F)$. Therefore, by Theorem 4.7 , the largest solution dominated by it has the same boundary trace, i.e. solves (4.22).

Next assume that condition (4.25) is satisfied. It is obvious that (4.25) is necessary for uniqueness. In addition, (4.25) implies that $U_{F} \leq u$ and consequently (4.24) implies (4.26). It is also clear that (b) implies the sufficiency part of (c).

Therefore it remains to prove statements (b) and (d). Let $u$ be the smallest solution dominating the subsolution $\max \left(V_{\nu}, U_{F}\right)$ and let $v$ be the largest solution dominated by $V_{v}+U_{F}$.

To establish (b) we must show that $u=v$. By (4.26) $v-u \leq V_{v}$. In addition the subsolution $v-u$ has trace zero on $\partial \Omega \backslash F$. Therefore

$$
v-u \leq \min \left(V_{v}, U_{F}\right)
$$

Let $\left\{N_{k}\right\}$ be a decreasing sequence of open sets converging to $F$ such that $N_{k+1} \Subset$ $N_{k}$. Assuming for a moment that $v$ is a finite measure, the trace of $V_{v}$ on $N_{k}$ is $v_{k}:=v \chi_{N_{k}}$ and it tends to zero as $k \rightarrow \infty$. Therefore, in this case,

$$
\min \left(V_{\nu}, U_{F}\right) \leq V_{\nu_{k}} \rightarrow 0
$$

and hence $u=v$. Of course this also implies uniqueness (statement (c)) in the case where $v$ is a finite measure.

In the general case we argue as follows. Let $v_{k}$ be the unique solution with boundary trace $\left(v_{k}^{\prime}, \bar{N}_{k}\right)$ where $v_{k}^{\prime}=v\left(1-\chi_{\bar{N}_{k}}\right)$. By taking a subsequence if necessary, we may assume that $\left\{v_{k}\right\}$ converges to a solution $v^{\prime}$. By (4.26),

$$
\max \left(V_{v_{k}^{\prime}}, U_{\bar{N}_{k}}\right) \leq v_{k} \leq V_{v_{k}^{\prime}}+U_{\bar{N}_{k}}
$$

and, by the previous part of the proof, $v_{k}$ is the largest solution dominated by $V_{v_{k}^{\prime}}+$ $U_{\bar{N}_{k}}$. We claim that if $w$ is a solution of (5.1) then

$$
V_{v} \leq w \leq V_{v}+U_{F} \Longrightarrow w \leq V_{v_{k}^{\prime}}+U_{\bar{N}_{k}} .
$$


Indeed,

$w \leq V_{v}+U_{F} \Longrightarrow w \leq V_{v_{k}^{\prime}}+V_{v_{k}}+U_{F} \Longrightarrow w \leq V_{v_{k}^{\prime}}+U_{\bar{N}_{k}}+U_{F} \Longrightarrow w \leq V_{v_{k}^{\prime}}+2 U_{\bar{N}_{k}}$.

Thus

which implies

$$
0 \leq w-V_{v_{k}^{\prime}} \leq 2 U_{\bar{N}_{k}}
$$

$$
w-V_{v_{k}^{\prime}} \leq U_{\bar{N}_{k}}
$$

because any solution (or subsolution) dominated by $2 U_{\bar{N}_{k}}$ is also dominated by $U_{\bar{N}_{k}}$.

Hence $v_{k} \geq v$ and consequently $v^{\prime} \geq v$.

By (4.20) $\bar{U}_{\bar{N}_{k}} \downarrow U_{F}$ and by definition $V_{v_{k}^{\prime}} \uparrow V_{\nu}$. Therefore

$$
\max \left(V_{v}, U_{F}\right) \leq v^{\prime} \leq V_{v}+U_{F} .
$$

Since $v$ is the largest solution dominated by $V_{v}+U_{F}$ and $v \leq v^{\prime}$ it follows that $v=v^{\prime}$.

Let $u_{k}$ be the unique solution with boundary trace $\left(v_{k}^{\prime}, k_{g}(F)\right)$. By (4.26),

$$
\max \left(V_{v_{k}^{\prime}}, U_{k_{g}(F)}\right) \leq u_{k} \leq V_{v_{k}^{\prime}}+U_{k_{g}(F)} .
$$

Since $u_{k} \leq u$ and $\left\{u_{k}\right\}$ increases (because $\left\{V_{v_{k}^{\prime}}\right\}$ increases) it follows that $u^{\prime}=$ $\lim u_{k} \leq u$. Furthermore,

$$
\max \left(V_{v}, U_{k_{g}(F)}\right) \leq u^{\prime} \leq V_{v}+U_{k_{g}(F)} .
$$

If (4.22) possesses a solution then condition (ii) holds. Therefore for any solution $w$ of $(5.1)$

$$
\max \left(V_{v}, U_{k_{g}(F)}\right) \leq w \Longrightarrow \max \left(V_{v}, U_{F}\right) \leq w .
$$

Hence $\max \left(V_{v}, U_{F}\right) \leq u^{\prime}$ and, as $u^{\prime} \leq u$ we conclude that $u^{\prime}=u$.

Finally, for every $\epsilon>0$,

$$
(1-\epsilon) V_{v_{k}^{\prime}}+\epsilon U_{k_{g}(F)} \leq u_{k}
$$

and consequently

$$
\begin{aligned}
& \left.v_{k}-u_{k} \leq V_{v_{k}^{\prime}}+U_{\bar{N}_{k}}-\left((1-\epsilon) V_{v_{k}^{\prime}}+\epsilon U_{k_{g}(F)}\right)\right) \\
& =U_{\bar{N}_{k}}-(1-\epsilon) U_{k_{g}(F)}+\epsilon V_{v_{k}^{\prime}} \leq U_{\overline{N_{k} \backslash F}}+U_{F}-(1-\epsilon) U_{k_{g}(F)}+\epsilon V_{v_{k}^{\prime}} \\
& \leq \epsilon\left(U_{F}+V_{v_{k}^{\prime}}\right) \rightarrow \epsilon\left(U_{F}+V_{v}\right) .
\end{aligned}
$$

This implies $u_{k}=v_{k}$ and hence $u=v$. This establishes statement (b) and hence the sufficiency in (c).

Finally we establish monotonicity. Let $v_{i}$ be the unique solution of (5.1) with boundary trace $\left(v_{i}, F_{i}\right),(\mathrm{i}=1,2)$. Then $v_{i}$ is the largest solution dominated by $V_{v_{i}}+$ $U_{F_{i}}(\mathrm{i}=1,2)$. The argument used in proving (4.30) yields

$$
V_{v_{1}} \leq w \leq V_{v_{1}}+U_{F_{1}} \Longrightarrow w \leq V_{\nu_{2}}+U_{F_{2}} .
$$

This implies $v_{1} \leq v_{2}$. 


\section{Equation with power nonlinearity in a Lipschitz domain}

In this section we study the trace problem and the associated boundary value problem for equation

$$
-\Delta u+|u|^{q-1} u=0
$$

in a Lipschitz bounded domain $\Omega$ and $q>1$. The main difference between the smooth cases and the Lipschitz case is the fact that the notion of critical exponent is pointwise. If $G$ is any domain in $\mathbb{R}^{N}$ we denote

$$
\mathcal{U}(G):=\{\text { the set of solutions (5.1) in } G\} .
$$

and $\mathcal{U}_{+}(G)=\{u \in \mathcal{U}(G): u \geq 0$ in $G\}$. Notice that any solution is at least $C^{3}$ in $G$ and any positive solution is $C^{\infty}$. The next result is proved separately by Keller [16] and Osserman [27].

Proposition 5.1. Let $q>1, \Omega \subset \mathbb{R}^{N}$ be any domain and $u \geq \in C(\Omega)$ be a weak solution of

$$
-\Delta u+A u^{q} \leq B \quad \text { in } \Omega .
$$

for some $A>0$ and $B \geq 0$. Then there exists $C_{i}(N, q)>0(i=1,2)$ such that

$$
u(x) \leq C_{1}\left(\frac{1}{\sqrt{A} \operatorname{dist}(x, \partial \Omega)}\right)^{2 /(q-1)}+C_{2}\left(\frac{B}{A}\right)^{1 / q} \quad \forall x \in \Omega .
$$

For a solution of (5.1) in $\Omega$ which vanishes on the boundary except at one point, we have a more precise estimate.

Proposition 5.2. Let $q>1, \Omega \subset \mathbb{R}^{N}$ be a bounded Lipschitz domain, $y \in \partial \Omega$ and $u \in \mathcal{U}_{+}(\Omega)$ is continuous in $\left.\bar{\Omega} \backslash\{y\}\right)$ and vanishes on $\partial \Omega \backslash\{y\}$. Then there exists $C_{3}(N, q, \Omega)>0$ and $\alpha \in(0,1]$ such that

$$
u(x) \leq C_{3}(\operatorname{dist}(x, \partial \Omega))^{\alpha}|x-y|^{-2 /(q-1)-\alpha} \quad \forall x \in \Omega .
$$

Furthermore $\alpha=1$ if $\Omega$ is a domain of class $W^{2, s}$ with $s>N$.

Note: A bounded domain $\Omega$ is of class $W^{2, s}$ if, for every $z \in \partial \Omega$, there exists a neighborhood $Q_{z}$ and a local set of coordinates $\zeta$ centered at $z$, such that $Q_{z} \cap \partial \Omega$ can be represented in the form $\zeta_{N}=F\left(\zeta_{1}, \ldots, \zeta_{N-1}\right)$ and $F$ belongs to $W^{2, s}$ in a neighborhood of the origin in $\mathbb{R}^{N-1}$.

Proof. By translation we can assume that $y=0$. Let $\tilde{u}$ be the extension of $u_{+}$by zero outside $\bar{\Omega} \backslash\{0\}$. Then it is a subsolution of (5.1) in $\mathbb{R}^{N} \backslash\{0\}$ (see [11] e.g.). Thus

$$
\tilde{u}(x) \leq C_{1}|x|^{-2 /(q-1)} \quad \forall x \neq 0,
$$

and, with the same estimate for $u_{-}$, we derive

$$
|u(x)| \leq C_{1}|x|^{-2 /(q-1)} \quad \forall x \in \Omega .
$$


Next we set, for $k>0, T_{k}[u]$ defined by $T_{k}[u](x)=k^{-2 /(q-1)} u\left(k^{-1} x\right)$, valid for any $x \in \Omega_{k}=k \Omega$. Then $u_{k}:=T_{k}[u]$ satisfies the same equation as $u$ in $\Omega_{k}$, is continuous in $\bar{\Omega}_{k} \backslash\{0\}$ and vanishes on $\partial \Omega_{k} \backslash\{0\}$. Then

$$
u_{k}(x) \leq C_{1}|x|^{-2 /(q-1)} \quad \forall x \in \Omega_{k},
$$

thus, by elliptic equation theory in uniformly Lipschitz domains, (which is the case if $k \geq 1$ )

$$
\left\|u_{k}\right\|_{C^{\alpha}\left(\Omega_{k} \cap\left(B_{7 / 4} \backslash B_{5 / 4}\right)\right)} \leq C\left\|u_{k}\right\|_{L^{\infty}\left(\Omega_{k} \cap\left(B_{2} \backslash B_{1}\right)\right)}=C_{2} .
$$

This implies

$$
\begin{aligned}
& \left|u\left(k^{-1} x^{\prime}\right)-u\left(k^{-1} z^{\prime}\right)\right| \leq C_{2} k^{-2 /(q-1)-\alpha}\left|x^{\prime}-z^{\prime}\right|^{\alpha} \\
& \forall(x, z) \in \Omega_{k} \times \Omega_{k}: 5 / 4 \leq\left|x^{\prime}\right|,\left|z^{\prime}\right| \leq 7 / 4 .
\end{aligned}
$$

Let $(x, z)$ in $\Omega \times \Omega$ close enough to 0 . First, if $5 / 7 \leq|x| /|z| \leq 7 / 5$ there exists $k \geq 1$ such that $5 / 4 \leq|k x|,|k z| \leq 7 / 4$. Then

$$
|u(x)-u(z)| \leq C_{3}|x|^{-2 /(q-1)-\alpha}|x-z|^{\alpha} .
$$

If we take in particular $x$ such that $z=\operatorname{Proj}_{\partial \Omega}(\mathrm{x})$ satisfies the above restriction, we derive

$$
u(x) \leq C_{3}|x|^{-2 /(q-1)-\alpha}(\operatorname{dist}(x, \partial \Omega))^{\alpha} .
$$

Because $\Omega$ is Lipschitz, it is easy to see that there exists $\beta \in(0,1 / 2)$ such that whenever dist $(x, \partial \Omega)=\left|x-\operatorname{Proj}_{\partial \Omega}(\mathrm{x})\right| \leq \beta|x|$, there holds

$$
5 / 7 \leq|x| /\left|\operatorname{Proj}_{\partial \Omega}(\mathrm{x})\right| \leq 7 / 5 .
$$

Next we suppose $\left|x-\operatorname{Proj}_{\partial \Omega}(\mathrm{x})\right|>\beta|x|$. Then, by the Keller-Osserman estimate,

$$
u(x) \leq C|x|^{-2 / q-1)-\alpha}|x|^{\alpha} \leq C \beta^{-\alpha}|x|^{-2 / q-1)-\alpha}\left|x-\operatorname{Proj}_{\partial \Omega}(\mathrm{x})\right|^{\alpha},
$$

which is (5.5). If we assume that $\partial \Omega$ is $W^{2, s}$, with $s>N$, then we can perform a change $W^{2, s}$ of coordinates near 0 with transforms $\partial \Omega \cap B_{R}(0)$ into $\mathbb{R}_{+}^{N} \cap B_{R}(0)$ and the equation into

$$
-\sum_{i, j} \frac{\partial}{\partial x_{i}}\left(a_{i j} \frac{\partial \tilde{u}}{\partial x_{j}}\right)+|\tilde{u}|^{q-1} \tilde{u}=0, \quad \text { in } \mathbb{R}_{+}^{N} \cap B_{R}(0) \backslash\{0\},
$$

where the $a_{i j}$ are the partial derivatives of the coordinates and thus belong to $W^{1, s}\left(B_{R}\right)$. By developping, $\tilde{u}$ satisfies

$$
-\sum_{i, j} a_{i j} \frac{\partial^{2} \tilde{u}}{\partial x_{i} \partial x_{j}}-\sum_{j} b_{j} \frac{\partial \tilde{u}}{\partial x_{j}}+|\tilde{u}|^{q-1} \tilde{u}=0 .
$$

Notice that, since $s>N$, the $a_{i j}$ are continuous while the $b_{i}$ are in $L^{s}$. The same regularity holds uniformly for the rescaled form of $\tilde{u}_{k}:=T_{k}[\tilde{u}]$. By the AgmonDouglis-Nirenberg estimates $\tilde{u}_{k}$ belongs to $W^{2, s}$. Since $s>N, \tilde{u}$ satisfies an uniform $C^{1}$ estimates, which implies that we can take $\alpha=1$. 


\subsection{Analysis in a cone}

The removability question for solutions of (5.1) near the vertex of a cone has been studied in [9], and we recall this result below.

If we look for separable solutions of (5.1) under the form $u(x)=u(r, \sigma)=$ $r^{\beta} \omega(\sigma)$, where $(r, \sigma) \in \mathbb{R}^{+} \times S^{N-1}$ are the spherical coordinates, one finds immediately $\beta=-2 /(q-1)$ and $\omega$ is a solution of

$$
-\Delta^{\prime} \omega-\lambda_{N, q} \omega+|\omega|^{q-1} \omega=0
$$

on $S^{N-1}$ with

$$
\lambda_{N, q}=\frac{2}{q-1}\left(\frac{2 q}{q-1}-N\right) .
$$

Thus, a solution of (5.1) in the cone $C_{S}=\left\{(r, \sigma): r>0, \sigma \in S \subset S^{N-1}\right\}$, vanishing on $\partial C_{S} \backslash\{0\}$, has the form $u(r, \sigma)=r^{-2 /(q-1)} \omega(\sigma)$ if and only if $\omega$ is a solution of (5.8) in $S$ which vanishes on $\partial S$. The next result [9, Propopsition 2.1] gives the the structure of the set of positive solutions of (5.8).

Proposition 5.3. Let $\lambda_{S}$ be the first eigenvalue of the Laplace-Beltrami operator $-\Delta^{\prime}$ in $W_{0}^{1,2}(S)$. Then

(i) If $\lambda_{S} \geq \lambda_{N, q}$ there exists no solution to (5.8) vanishing on $\partial S$.

(ii) If $\lambda_{S}<\lambda_{N, q}$ there exists a unique positive solution $\omega=\omega_{S}$ to (5.8) vanishing on $\partial S$. Furthermore $S \subset S^{\prime} \Longrightarrow \omega_{S} \leq \omega_{S^{\prime}}$.

The following is a consequence of Proposition 5.3.

Proposition 5.4. [9] Assume $\Omega$ a bounded domain with a purely conical part with vertex 0 , that is

$$
\Omega \cap B_{r_{0}}(0)=C_{S} \cap B_{r_{0}}(0)=\left\{x \in \cap B_{r_{0}}(0) \backslash\{0\}: x /|x| \in S\right\} \cup\{0\}
$$

and that $\partial \Omega \backslash\{0\}$ is smooth. Then, if $\lambda_{S} \geq \lambda_{N, q}$, any solution $u \in \mathcal{U}(\Omega)$ which is continuous in $\bar{\Omega} \backslash\{0\}$ and vanishes on $\partial \Omega \backslash\{0\}$ is identically 0 .

Remark 5.5. If $S \subset S^{N-1}$ is a domain and $\lambda_{S}$ the first eigenvalue of the LaplaceBeltrami operator $-\Delta^{\prime}$ in $W_{0}^{1,2}(S)$ we denote by $\tilde{\alpha}_{S}$ and $\alpha_{S}$ the positive root and the absolute value of the negative root respectively, of the equation

$$
X^{2}+(N-2) X-\lambda_{S}=0 \text {. }
$$

Thus

$$
\begin{aligned}
& \tilde{\alpha}_{S}=\frac{1}{2}\left(2-N+\sqrt{(N-2)^{2}+4 \lambda_{S}}\right), \\
& \alpha_{S}=\frac{1}{2}\left(N-2+\sqrt{(N-2)^{2}+4 \lambda_{S}}\right) .
\end{aligned}
$$


It is straightforward that

$$
\lambda_{S} \geq \lambda_{N, q} \Longleftrightarrow \alpha_{S} \geq \frac{2}{q-1},
$$

and, in case of equality, the exponent $q=q_{S}$ satisfies $q_{S}=1+2 / \alpha_{S}$.

From the previous remarks it follows that, if $C_{S}$ is the cone with vertex at the origin and "opening" $S \subset S^{N-1}$, we have

$$
K^{C_{S}}(x, 0)=|x|^{-\alpha_{S}} \omega_{S}(\sigma), \quad \rho(x)=|x|^{\tilde{\alpha}_{S}} \omega_{S}(\sigma),
$$

where $\omega_{S}$ is as in Proposition 5.3.

Combining the removability result with the admissibility condition Theorem 3.10 , we obtain the following.

Theorem 5.6. The problem

$$
\begin{aligned}
& -\Delta u+|u|^{q-1} u=0 \text { in } C_{S}, \\
& \quad u \in C\left(\bar{C}_{S} \backslash\{0\}\right), \quad u=0 \text { on } \partial C_{S} \backslash\{0\}
\end{aligned}
$$

possesses a non-trivial solution if and only if

$$
1<q<q_{S}=1+2 / \alpha_{S} .
$$

Under this condition the following statements hold.

(a) For every $k \neq 0$ there exists a unique solution $v_{k}$ of (5.1) with boundary trace $k \delta_{0}$. In addition we have

$$
v_{k} / v_{1}(x) \rightarrow k \quad \text { uniformly as } x \rightarrow 0
$$

(b) Equation (5.1) possesses a unique solution $U$ in $C_{S}$ such that $\mathcal{S}(U)=\{0\}$ and its trace on $\partial C_{S} \backslash\{0\}$ is zero. This solution satisfies

$$
|x|^{\frac{2}{q-1}} U(x)=U(x /|x|)=\omega_{S}(x /|x|)
$$

and

$$
U=v_{\infty}:=\lim _{k \rightarrow \infty} v_{k}
$$

Proof. (a) By (5.11),

$$
\int_{C_{S} \cap B_{1}} K^{q}(x, 0) \rho(x) d x \leq C \int_{0}^{1} r^{\tilde{\alpha}_{S}-q \alpha_{S}+N-1} d r<\infty,
$$

since

$$
\tilde{\alpha}_{S}-q \alpha_{S}+N-1=1-(q-1) \alpha_{S}>-1 .
$$


Thus $q$ is admissible for $C_{S} \cap B_{1}$ at 0 . By Theorem 3.10 , for every $k \in \mathbb{R}$, there exists a unique solution of (5.1) with boundary trace $k \delta_{0}$.

Observe that, for every $a, j>0, \tilde{v}_{j}(x):=a^{2 /(q-1)} v_{j}(a x)$ is a solution of (5.1) in $C_{S}$. This solution has boundary trace $k \delta_{0}$ where $k=a^{2 /(q-1)} j$. Because of uniqueness, $\tilde{v}_{j}=v_{k}$. Thus

$$
v_{k}(x)=a^{2 /(q-1)} v_{j}(a x), \quad k=a^{2 /(q-1)} j .
$$

This implies (5.13).

(b) Let $w$ be a solution in $C_{S}$ such that $\mathcal{S}(w)=\{0\}$ and its trace on $\partial C_{S} \backslash\{0\}$ is zero. We claim that

$$
w \geq v_{\infty}:=\lim k \rightarrow \infty v_{k}
$$

Indeed, for every $S^{\prime} \Subset S, k>0$,

$$
\int_{a S^{\prime}} w d \omega_{a} \rightarrow \infty, \quad \limsup \int_{a S^{\prime}} v_{k} d \omega_{a}<\infty \text { as } a \rightarrow 0
$$

where $d \omega_{a}$ denotes the harmonic measure for a bounded Lipschitz domain $\Omega_{a}$ such that $a S^{\prime} \subset \partial \Omega_{a}$ and $\Omega_{a} \uparrow C_{S}$. Therefore, using the classical Harnack inequality up to the boundary, $w / v_{k} \rightarrow \infty$ as $|x| \rightarrow 0$ in $C_{S^{\prime}}$. In addition, either by Hopf's maximum principle (if $S$ is smooth) or by the boundary Harnack principle (if $S$ is merely Lipschitz),

$$
c^{-1} v_{1} \leq w \leq c v_{1} \text { in } C_{S \backslash S^{\prime}} .
$$

This inequality together with (5.16) yields,

$$
c^{-1} v_{k} \leq w \leq c v_{k} \text { in } C_{S \backslash S^{\prime}}
$$

with $c$ independent of $k$. Therefore $c^{-1} v_{k} \leq w$ in $C_{S}$. If $1 / c>k / c j>1$ then $\frac{k}{j} v_{j} \leq v_{k} \leq c w$ and consequently $v_{j}<w$. Here we used the fact that $\frac{k}{j} v_{j}$ is a subsolution with boundary trace $k \delta_{0}$.

Let $U_{0}$ be the maximal solution with trace 0 on $\partial C_{S} \backslash\{0\}$ and singular boundary point at 0 . Then

$$
U_{0}(x)=a^{2 /(q-1)} U_{0}(a x) \quad \forall a>0, x \in C_{S},
$$

because $a^{2 /(q-1)} U_{0}(a x)$ is again a solution which dominates every solution with trace 0 on $\partial C_{S} \backslash\{0\}$ and singular boundary point at 0 . Hence,

$$
U_{0}(x)=|x|^{-2 /(q-1)} U_{0}(x /|x|)=|x|^{-2 /(q-1)} \omega_{S}(x /|x|) .
$$

The second equality follows from the uniqueness part in Proposition 5.3 since the function $x \rightarrow U_{0}(x /|x|)$ is continuous in $\bar{S}$ and vanishes on $\partial S$.

Inequality (5.17) implies that $v_{\infty}$ is the minimal positive solution such that $\mathcal{S}(w)=\{0\}$ and its trace on $\partial C_{S} \backslash\{0\}$ is zero. Using this fact we prove in the same way that $v_{\infty}$ satisfies

$$
v_{\infty}(x)=|x|^{-2 /(q-1)} v_{\infty}(x /|x|)=|x|^{-2 /(q-1)} \omega_{S}(x /|x|) .
$$

This implies (5.15) and the uniqueness in statement (b). 
In the next theorem we describe the precise asymptotic behavior of solutions in a conical domain with mass concentrated at the vertex.

Theorem 5.7. Let $C_{S}$ be a cone with vertex 0 and opening $S \subset S^{N-1}$ and assume that $1<q<q_{S}=1+2 / \alpha_{S}$. Denote by $\phi_{S}$ the first eigenfunction of $-\Delta^{\prime}$ in $W_{0}^{1,2}(S)$ normalized by $\max \phi_{S}=1$. Then the function

$$
\Phi_{S}=x^{-\alpha} \phi_{S}(x /|x|),
$$

with $\alpha_{S}$ as in (5.10), is harmonic in $C_{S}$ and vanishes on $\partial C_{S} \backslash\{0\}$. Thus there exists $\gamma>0$ such that the boundary trace of $\Phi_{S}$ is the measure $\gamma \delta_{0}$. Put $\Phi_{1}:=\frac{1}{\gamma} \Phi_{S}$.

Let $r_{0}>0$ and denote $\Omega_{S}=C_{S} \cap B_{r_{0}}(0)$. For every $k \in \mathbb{R}$, let $u_{k}$ be the unique solution of (5.1) in $\Omega_{S}$ with boundary trace $k \delta_{0}$. Then

$$
u_{k}(x)=k \Phi_{1}(x)(1+o(1)) \quad \text { as } x \rightarrow 0 .
$$

If $v_{k}$ is the unique solution of (5.1) in $C_{S}$ with boundary trace $k \delta_{0}$ then

$$
u_{k} / v_{k} \rightarrow 1 \text { and } v_{k} /\left(k \Phi_{1}\right) \rightarrow 1 \text { as } x \rightarrow 0 .
$$

The function $u_{\infty}=\lim _{k \rightarrow \infty} u_{k}$ is the unique positive solution of (5.1) in $\Omega_{S}$ which vanishes on $\partial \Omega_{S} \backslash\{0\}$ and is strongly singular at 0 (i.e., 0 belongs to its singular set). Its asymptotic behavior at 0 is given by,

$$
u_{\infty}(x)=|x|^{-\frac{2}{q-1}} \omega_{S}(x /|x|)(1+o(1)) \quad \text { as } x \rightarrow 0 .
$$

Proof. Step 1: Construction of a fundamental solution. Put

$$
\Phi(x)=|x|^{-\alpha_{S}} \phi_{S}(x /|x|), \quad \tilde{\Phi}(x)=|x|^{\tilde{\alpha}_{S}} \phi_{S}(x /|x|)
$$

with $\alpha_{S}, \tilde{\alpha}_{S}$ as in (5.10). Then $\Phi$ and $\tilde{\Phi}$ are harmonic in $C_{S}$, $\Phi$ vanishes on $\partial C_{S} \backslash\{0\}$ and $\tilde{\Phi}$ vanishes on $\partial C_{S}$. Furthermore, since $q<1+2 / \alpha_{S}$,

$$
\int_{C_{S} \cap B_{1}(0)} \Phi^{q} \rho d x<\infty .
$$

Therefore the boundary trace of $\Phi$ is a bounded measure concentrated at the vertex of $C_{S}$, which means that the trace is $\gamma \delta_{0}$ for some $\gamma>0$. (Here $\delta_{0}$ denotes the Dirac measure on $\partial C_{S}$ concentrated at the origin.)

The function

$$
\Psi(x)=\frac{1}{\gamma}\left(\Phi(x)-r_{0}^{\tilde{\alpha}_{S}-\alpha_{S}} \tilde{\Phi}(x)\right)
$$

is harmonic and positive in $\Omega_{S}$ and vanishes on $\partial \Omega_{S} \backslash\{0\}$. Its boundary trace is $\delta_{0}$. 
Step 2: Weakly singular behaviour. By Theorem 3.10, for any $k \geq 0$, there exists a unique function $u_{k} \in L_{\rho}^{q}\left(\Omega_{S}\right)$ with trace $k \delta_{0}$ and by (3.8)

$$
u_{k}(x)=k \Psi(x)-\mathbb{G}\left[\left|u_{k}\right|^{q}\right] .
$$

Since $|x|^{\alpha} s u_{k}$ is bounded, we set

$$
v(t, \sigma)=r^{\alpha} u_{k}(r, \sigma), \quad t=-\ln r .
$$

Then $v$ satisfies

$$
v_{t t}+\left(2 \alpha_{S}+2-N\right) v_{t}+\lambda_{S} v+\Delta^{\prime} v-e^{\left(\alpha_{S}(q-1)-2\right) t}|v|^{q-1} v=0
$$

in $D_{S, t_{0}}:=\left[t_{0}, \infty\right) \times S$ (with $\left.t_{0}:=-\ln r_{0}\right)$ and vanishes on $\left[t_{0}, \infty\right) \times \partial S$. Since $0 \leq u_{k}(x) \leq k \Psi(x), v$ is uniformly bounded, and, since $\alpha_{S}(q-1)-2<0, v(t,$. is uniformly bounded in $C^{\alpha}(\bar{S})$ for some $\alpha \in(0,1)$. Furthermore, $\nabla^{\prime} v(t,$.$) (by$ definition $\nabla^{\prime}$ is the covariant gradient on $\left.S^{N-1}\right)$ is bounded in $L^{2}(S)$, independently of $t$. Set

$$
y(t)=\int_{S} v(t, \sigma) \phi_{S} d V(\sigma), \quad F(t)=\int_{S}\left(|v|^{q-1} v\right)(t, \sigma) \phi_{S} d V(\sigma) .
$$

From (5.24), it follows

$$
\frac{d}{d t}\left(e^{\left(2 \alpha_{S}+2-N\right) t} y^{\prime}\right)=e^{\left((q+1) \alpha_{S}-N\right) t} F
$$

where $d V$ is the volume measure on $S^{N-1}$. By (5.10), $\gamma:=2 \alpha_{S}+2-N>0$, then

$$
y^{\prime}(t)=e^{-\gamma\left(t-t_{0}\right)} y^{\prime}\left(t_{0}\right)+e^{-\gamma t} \int_{t_{0}}^{t} e^{\left((q+1) \alpha_{S}-N\right) s} F(s) d s,
$$

and

$$
\left|y^{\prime}(t)\right| \leq c_{1} e^{-\gamma\left(t-t_{0}\right)}+c_{2} e^{\left(\alpha_{S}(q-1)-2\right) t} .
$$

This implies that there exists $k^{*} \in \mathbb{R}^{+}$such that

$$
\lim _{t \rightarrow \infty} y(t)=k^{*} .
$$

Next we use the fact that the following Hilbertian decomposition holds

$$
L^{2}(S)=\oplus_{k=1}^{\infty} \operatorname{ker}\left(-\Delta^{\prime}-\lambda_{k} I\right)
$$

where $\lambda_{k}$ is the $k$-th eigenvalue of $-\Delta^{\prime}$ in $W_{0}^{1,2}(S)$ (and $\left.\lambda_{S}=\lambda_{1}\right)$. Let $\tilde{v}$ and $\tilde{F}$ be the projections of $v$ and $|v|^{q-1} v$ onto $\operatorname{ker}\left(-\Delta^{\prime}-\lambda_{S} I\right)^{\perp}$. Since

$$
\tilde{v}_{t t}+\left(2 \alpha_{S}+2-N\right) \tilde{v}_{t}+\lambda_{S} \tilde{v}+\Delta^{\prime} \tilde{v}-e^{\left(\alpha_{S}(q-1)-2\right) t} \tilde{F}=0
$$


we obtain, by multiplying by $\tilde{w}$ and integrating on $S$,

$$
V^{\prime \prime}+\left(2 \alpha_{S}+2-N\right) V^{\prime}-\left(\lambda_{2}-\lambda_{S}\right) V+e^{\left(\alpha_{S}(q-1)-2\right) t} \Phi \geq 0,
$$

where $V(t)=\|\tilde{v}(t, .)\|_{L^{2}(S)}$ and $\Phi(t)=\|\tilde{F}(t, .)\|_{L^{2}(S)}$. The associated o.d.e.

$$
z^{\prime \prime}+\left(2 \alpha_{S}+2-N\right) z^{\prime}-\left(\lambda_{2}-\lambda_{S}\right) z+e^{\left(\alpha_{S}(q-1)-2\right) t} \Phi=0,
$$

admits solutions under the form

$$
z(t)=a_{1} e^{-\mu_{1} t}+a_{2} e^{\mu_{2} t}+d(t) e^{\left(\alpha_{S}(q-1)-2\right) t}
$$

where $-\mu_{1}$ and $\mu_{2}$ are respectively the negative and the positive roots of

$$
X^{2}+\left(2 \alpha_{S}+2-N\right) X-\left(\lambda_{2}-\lambda_{S}\right)=0,
$$

and $|d(t)| \leq c \Phi$ if $\alpha_{S}(q-1)-2 \neq-\mu_{1}$, or $|d(t)| \leq c t^{1} \Phi$ if $\alpha_{S}(q-1)-2=-\mu_{1}$. Applying the maximum principle, to (5.26), we derive

$$
\|\tilde{v}(t, .)\|_{L^{2}(S)} \leq\left\|\tilde{v}\left(t_{0}, .\right)\right\|_{L^{2}(S)} e^{-\mu_{1}\left(t-t_{0}\right)}+d(t) e^{\left(\alpha_{S}(q-1)-2\right) t} \quad \forall t \geq t_{0} .
$$

By the standard elliptic regularity results in Lipschitz domains [10], we obtain from (5.27), for any $t>t_{0}+1$,

$$
\|\tilde{v}(t, .)\|_{C^{\alpha}(S)} \leq c_{1}\|\tilde{v}\|_{L^{2}((t-1, t+1) \times S)}+c_{2}\left\|e^{\left(\alpha_{S}(q-1)-2\right) s} \tilde{F}\right\|_{L^{\infty}((t-1, t+1) \times S)},
$$

for some $\alpha \in(0,1]$ depending of the regularity of $\partial S$. Thus

$$
\|\tilde{v}(t, .)\|_{C^{\alpha}(S)} \leq c e^{-\mu_{1} t}+c^{\prime} t e^{\left(\alpha_{S}(q-1)-2\right) t} .
$$

Combining (5.25) and (5.29) we obtain that

$$
|x|^{\alpha} u_{k}(x)-k^{*} \phi_{S}(x /|x|) \rightarrow 0 \quad \text { as } x \rightarrow 0
$$

in $C^{\alpha}(S)$. Furthermore $0 \leq k^{*} \leq k$.

Step 3: Identification of $k^{*}$. Let $\left\{\Omega_{n}\right\}$ be a Lipschitz exhaustion of $\Omega_{S}$ and denote by $\omega_{n}$ (respectively $\omega$ ) the harmonic measure on $\partial \Omega_{n}$ (respectively $\partial \Omega_{S}$ ). By Proposition 3.8

$$
\lim _{n \rightarrow \infty} \int_{\partial \Omega_{n}} u_{k} d \omega_{n}=k .
$$

On the other hand, by (5.30),

$$
u_{k} /\left(k^{*}|x|^{-\alpha_{S}} \phi_{S}\right) \rightarrow 1 \text { as } x \rightarrow 0 .
$$


Hence

$$
\begin{aligned}
\lim _{n \rightarrow \infty} \int_{\partial \Omega_{n}} u_{k} d \omega_{n} & =k^{*} \lim _{n \rightarrow \infty} \int_{\partial \Omega_{n}}|x|^{-\alpha_{S}} \phi_{S} d \omega_{n} \\
& =k^{*} \gamma \lim _{n \rightarrow \infty} \int_{\partial \Omega_{n}} \Phi_{1} d \omega_{n}=k^{*} \gamma
\end{aligned}
$$

Thus

$$
k=k^{*} \gamma
$$

This and (5.30) imply (5.19).

Further,

$$
u_{k} \leq v_{k} \leq k \Phi_{1}
$$

since $\Phi_{1}$ is harmonic in $C_{S}$. Therefore (5.19) implies (5.20).

Step 4: Study when $k \rightarrow \infty$. By Theorem 5.6, equation (5.1) possesses a unique solution $U$ in $C_{S}$ such that $U=0$ on $\partial C_{S} \backslash\{0\}$ and $U$ has strong singularity at the vertex, i.e., $0 \in \mathcal{S}(U)$. By (5.14) and (5.15) this solution satisfies

$$
U=v_{\infty}:=\lim _{k \rightarrow \infty} v_{k}=|x|^{-\frac{2}{q-1}} \omega_{S}
$$

Let $V$ be the maximal solution in $\Omega_{S}$ vanishing on $\partial \Omega_{S} \backslash\{0\}$. Its extension by zero to $C_{S}$ is a subsolution and consequently, $V \leq U$.

Let $w$ be the unique solution of (5.1) in $\Omega_{S}$ such that $w=U$ on $\partial \Omega_{S} \cap B_{r_{0}}(0)$ and $w=0$ on the remaining part of the boundary. Then $w<U$ so that $U-w$ is a subsolution of (5.1) in $\Omega_{S}$ which vanishes on $\partial \Omega_{S} \backslash\{0\}$. Therefore $U-w \leq V$. Thus

$$
U-w \leq V \leq U \text { and } U / V \rightarrow 1 \text { as } x \rightarrow 0 .
$$

Let $u$ be an arbitrary positive solution in $\Omega_{S}$ vanishing on $\partial \Omega_{S} \backslash\{0\}$. Denote by $u^{*}$ its extension by zero to $C_{S}$. Then $u^{*}$ is a subsolution and, by Theorem 4.3 , there exists a solution $\bar{u}$ of (5.1) in $C_{S}$ which is the smallest solution dominating $u^{*}$. The solution $\bar{u}$ can be obtained from $u^{*}$ as follows. Let $\left\{r_{n}\right\}$ be a sequence decreasing to zero, $r_{1}<r_{0}$, and denote

$$
D_{n}=C_{S} \backslash B_{r_{n}}(0), \quad h_{n}=u^{*}\left\lfloor_{\partial D_{n}} .\right.
$$

Let $w_{n}$ be the solution of (5.1) in $D_{n}$ such that $w_{n}=h_{n}$ on the boundary. Then $\left\{w_{n}\right\}$ increases and

$$
\bar{u}=\lim w_{n} .
$$

If $u$ has strong singularity at the origin then, of course, the same is true with respect to $\bar{u}$ and consequently, by Theorem 5.6,

$$
\bar{u}=U \text {. }
$$

In the remaining part of the proof we assume only (5.35) and show that this implies $u=V$. 
Let $z$ be the solution of (5.1) in $\Omega_{S}$ such that $z=U$ on $\partial \Omega_{S} \cap \partial B_{r_{0}}$ and 0 on $\partial \Omega_{S} \cap \partial C_{S}$. Then $u+z$ is a supersolution in $\Omega_{S}$. Let

$$
\Omega_{n}=\Omega_{S} \backslash B_{r_{n}}(0)=D_{n} \cap B_{r_{0}}(0) .
$$

The trace of $u+z$ on $\partial \Omega_{n}$ is given by

$$
f_{n}= \begin{cases}U & \text { on } \partial \Omega_{n} \cap \partial B_{r_{0}} \\ h_{n}+z & \text { on } \partial \Omega_{n} \backslash \partial B_{r_{0}} .\end{cases}
$$

Since $U=\bar{u} \geq u^{*}$ we have $f_{n} \geq h_{n}$. Therefore, if $\tilde{w}_{n}$ is the solution of (5.1) in $\Omega_{n}$ such that $\tilde{w}_{n}=f_{n}$ on the boundary then

$$
w_{n} \leq \tilde{w}_{n} \leq u+z \text { in } \Omega_{n} .
$$

Hence, by (5.34),

$$
U \leq u+z .
$$

Since $z \rightarrow 0$ as $x \rightarrow 0$, it follows that

$$
\lim \sup U / u \leq 1 \text { as } x \rightarrow 0 .
$$

Since $u<V$, (5.33) implies that

$$
\liminf U / u \geq 1 \text { as } x \rightarrow 0 .
$$

Therefore $U / u \rightarrow 1$ as $x \rightarrow 0$ and consequently, by (5.33) and the maximum principle, $u=V$. This proves the uniqueness stated in the last part of the theorem and (5.33) implies (5.21).

Corollary 5.8. Suppose that $u$ is a positive solution of (5.1) in $\Omega_{S}$ which vanishes on $\partial \Omega_{S} \backslash\{0\}$ and

$$
\sup _{\Omega_{S}}|x|^{\alpha_{S}} u=\infty .
$$

Then $u=u_{\infty}$.

Proof. Let $\bar{u}$ be as in (5.34). Since $\bar{u} \geq u$ it follows that

$$
\sup _{\Omega_{S}}|x|^{\alpha_{S}} \bar{u}=\infty .
$$

By Theorem 5.6 $\bar{u}=U$. The last part of the proof shows that $u=u_{\infty}$.

As a consequence of Theorem 5.7 we obtain the classification of positive solutions of (5.1) in conical domains with isolated singularity located at the vertex. In the case of a half space such a classification was obtained in [11]. 
Theorem 5.9. Let $C_{S}$ be as in Theorem 5.7, $\Omega_{s}=C_{S} \cap B_{r_{0}}(0)$ for some $r_{0}>0$ and $1<q<q_{S}=1+2 / \alpha_{S}$. If $u \in C\left(\bar{\Omega}_{S} \backslash\{0\}\right)$ is a positive solution of (5.1) vanishing on $\partial C_{S} \cap B_{r_{0}}(0) \backslash\{0\}$, the following alternative holds:

Either

(i) $\lim \sup _{x \rightarrow 0}|x|^{-\tilde{\alpha}_{S}} u(x)<\infty$ and thus $u \in C\left(\bar{\Omega}_{s}\right)$.

or

(ii) there exist $k>0$ such that (5.19) holds

or

(iii) (5.21) holds.

Proof. Let $u_{\epsilon}$ be the solution of (5.1) in $\Omega_{S, \epsilon}=\Omega_{S} \backslash B_{\epsilon}(0)$ with boundary data $u$ on $\Omega_{S, \epsilon} \cap \partial B_{\epsilon}(0)$ and zero on $\partial \Omega_{S, \epsilon} \backslash \partial B_{\epsilon}(0)$. Then

$$
0 \leq u_{\epsilon} \leq u \leq u_{\epsilon}+Z(x) \quad \forall x \in \Omega_{S, \epsilon},
$$

where $Z$ is harmonic in $\Omega_{S}$, vanishes on $\partial \Omega_{S} \backslash \partial B_{r_{0}}(0)$ and coincides with $u$ on $C_{S} \cap \partial B_{r_{0}}(0)$. Furthermore $0<\epsilon<\epsilon^{\prime} \Longrightarrow u_{\epsilon} \leq u_{\epsilon^{\prime}}$ in $\Omega_{s, \epsilon^{\prime}}$. Thus $u_{\epsilon}$ converges, as $\epsilon \rightarrow 0$, to a solution $\tilde{u}$ of (5.1) which vanishes on $\partial \Omega_{S} \backslash\{0\}$ and satisfies

$$
0 \leq \tilde{u}(x) \leq u(x) \leq \tilde{u}(x)+Z(x) \quad \forall x \in \Omega_{s} .
$$

If

$$
\limsup _{x \rightarrow 0}|x|^{\alpha} S \tilde{u}(x)<\infty,
$$

it follows from Theorem 5.7-Step 2, that there exists $k^{*} \geq 0$ such that

$$
\tilde{u}(x)=k^{*}|x|^{-\alpha} \phi_{S}(x /|x|)(1+o(1)) \quad \text { as } x \rightarrow 0 .
$$

If $k^{*}>0$ then $u$ satisfies (ii). If $k^{*}=0$, it is straightforward to see that, for any $\epsilon>0, \tilde{u}(x) \leq \epsilon|x|^{-\alpha_{S}}$. Thus

$$
u(x) \leq Z(x)=c|x|^{\tilde{\alpha}_{S}} \phi_{S}(x /|x|)(1+o(1)) \quad \text { as } x \rightarrow 0,
$$

by standard expansion of harmonic functions at 0 .

Finally, if

$$
\limsup _{x \rightarrow 0}|x|^{\alpha} S \tilde{u}(x)=\infty,
$$

then, by Corollary 5.8, $\tilde{u}=u_{\infty}$ and consequently, by Theorem 5.7, $\tilde{u}-$ and therefore $u$ - satisfies (5.21). 


\subsection{Analysis in a Lipschitz domain}

In a general Lipschitz bounded domain tangent planes have to be replaced by asymptotic cones, and these asymptotic cones can be inner or outer.

Definition 5.10. Let $\Omega$ be a bounded Lipschitz domain and $y \in \partial \Omega$. For $r>0$, we denote by $\mathcal{C}_{y, r}^{I}$ (respectively $\mathcal{C}_{y, r}^{O}$ ) the set of all open cones $C_{s, y}$ with vertex at $y$ and smooth opening $S \subset \partial B_{1}(y)$ such that $C_{s, y} \cap B_{r}(y) \subset \Omega$ (respectively $\left.\Omega \cap B_{r}(y) \subset C_{s, y}\right)$. Further we denote

$$
C_{y, r}^{I}:=\bigcup\left\{C_{S, y}: C_{S, y} \in \mathcal{C}_{y, r}^{I}\right\}, \quad C_{y, r}^{O}:=\bigcap\left\{C_{S, y}: C_{S, y} \in \mathcal{C}_{y, r}^{O}\right\}
$$

and

$$
C_{y}^{I}:=\bigcup_{r>0} C_{y, r}^{I}, \quad C_{y}^{O}:=\bigcap_{r>0} C_{y, r}^{O} .
$$

The cone $C_{y}^{I}$ (respectively $C_{y}^{O}$ ) is called the limiting inner cone (respectively outer cone) at $y$. Finally we denote

$$
\begin{aligned}
S_{y, r}^{I} & :=C_{y, r}^{I} \cap \partial B_{1}(y), & S_{y, r}^{O} & :=C_{y, r}^{O} \cap \partial B_{1}(y), \\
S_{y}^{I} & :=C_{y}^{I} \cap \partial B_{1}(y), & S_{y}^{O} & :=C_{y}^{O} \cap \partial B_{1}(y) .
\end{aligned}
$$

Remark 5.11. In this definition, we identify $\partial B_{1}(y)$ with the manifold $S^{N-1}$. Notice that the following monotonicity holds

$$
0<s<r \Longrightarrow\left\{\begin{array}{l}
C_{y, r}^{I} \subset C_{y, s}^{I} \\
C_{y, s}^{O} \subset C_{y, r}^{I} .
\end{array}\right.
$$

Definition 5.12. If $C_{S}$ is a cone with vertex $y$ and opening $S$ and if $\lambda_{S}$ is the first eigenvalue of $-\Delta^{\prime}$ in $W_{0}^{1,2}(S)$, we denote

$$
\alpha_{S}=\frac{1}{2}\left(N-2+\sqrt{(N-2)^{2}+4 \lambda_{S}}\right) \text {, and } q_{S}=1+2 / \alpha_{S} .
$$

Thus $q_{S}$ is the critical value for the cone $C_{S}$ at its vertex.

Remark 5.13. As $r \mapsto S_{y, r}^{I}$ is nondecreasing, it follows that $r \mapsto \lambda_{S_{y, r}^{I}}$ is nonincreasing and consequently $r \mapsto q_{S_{y, r}^{I}}$ is nondecreasing. It is classical that

$$
\lim _{r \rightarrow 0} \lambda_{S_{y, r}^{I}}=\lambda_{S_{y}^{I}} \text {. }
$$

A similar observation holds with respect to $S_{y, r}^{O}$ if we interchange the terms "nondecreasing" and "nonincreasing". In particular

$$
\lim _{r \rightarrow 0} \lambda_{S_{y, r}^{O}}=\lambda_{S_{y}^{O}} .
$$

In view of (5.46) we conclude that,

$$
\lim _{r \rightarrow 0} q_{S_{y, r}^{I}}=q_{S_{y}^{I}}, \quad \lim _{r \rightarrow 0} q_{S_{y, r}^{O}}=q_{S_{y}^{O}} .
$$

We also need the following notation: 
Definition 5.14. Let $\Omega$ be a bounded Lipschitz domain. For every compact set $E \subset \partial \Omega$ denote,

$$
q_{E}^{*}=\lim _{r \rightarrow 0} \inf \left\{q_{S_{z, r}^{I}}: z \in \partial \Omega, \operatorname{dist}(z, E)<r\right\}
$$

If $E$ is a singleton, say $\{y\}$, we replace $q_{E}^{*}$ by $q_{y}^{*}$.

Remark 5.15. For a cone $C_{S}$ with vertex $y, q_{y}^{*} \leq q_{S}$. However if $C_{S}$ is contained in a half space then $q_{y}^{*}=q_{S}$. On the other hand, if $C_{S}$ strictly contains a half space then $q_{y}^{*}<q_{S}$.

If $\Omega$ is the complement of a bounded convex domain then, for every $y \in \partial \Omega$,

$$
q_{y}^{*}=(N+1) /(N-1) \text {. }
$$

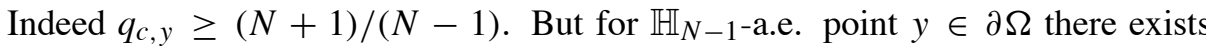
a tangent plane and consequently $q_{c, y}=(N+1) /(N-1)$. This readily implies (5.51).

Since $\Omega$ is Lipschitz, there exists $r_{\Omega}>0$ such that, for every $r \in\left(0, r_{\Omega}\right)$ and every $z \in \partial \Omega$, there exists a cone $C$ with vertex at $z$ such that $C \cap B_{r}(z) \subset \bar{\Omega}$. Denote

$$
a(r, y):=\inf \left\{q_{S_{z, r}^{I}}: z \in \partial \Omega \cap B_{r}(y)\right\} \quad \forall r \in\left(0, r_{\Omega}\right), y \in \partial \Omega .
$$

Then,

$$
\begin{aligned}
q_{E}^{*} & :=\liminf _{r \rightarrow 0}\{a(r, y): y \in E\} \\
& \leq \inf \left\{\lim _{r \rightarrow 0} a(r, y): y \in E\right\}=\inf \left\{q_{y}^{*}: y \in E\right\} .
\end{aligned}
$$

Indeed, the monotonicity of the function $r \mapsto q_{S_{y, r}^{I}}$ (for each fixed $y \in \partial \Omega$ ) implies

$$
q_{y}^{*}=\lim _{r \rightarrow 0} a(r, y)=\sup _{0<r<r_{\Omega}} a(r, y)
$$

As

$$
q_{E}^{*}=\lim _{r \rightarrow 0} \inf \{a(r, y): y \in E\}
$$

inequality (5.52) follows immediately from (5.53).

Finally we observe that, if $E$ is a compact subset of $\partial \Omega$ then

$$
(E)_{r}:=\{z \in \partial \Omega: \operatorname{dist}(z, E) \leq r\} \Longrightarrow q_{(E)_{r}}^{*} \uparrow q_{E}^{*} \text { as } r \downarrow 0 .
$$

In order to deal with boundary value problems in a general Lipschitz domain $\Omega$ we must study the question of q-admissibility of $\delta_{y}, y \in \partial \Omega$. This question is addressed in the following: 
Theorem 5.16. If $y \in \partial \Omega$ and $1<q<q_{S_{y}^{I}}:=1+2 / \alpha_{S_{y}^{I}}$ then

$$
\int_{\Omega} K^{q}(x, y) \rho(x) d x<\infty .
$$

Furthermore, if $E$ is a compact subset of $\partial \Omega$ and $1<q<q_{E}^{*}$ then, there exists $M>0$ such that,

$$
\int_{\Omega} K^{q}(x, y) \rho(x) d x \leq M \quad \forall y \in E .
$$

Proof. We recall some sharp estimates of the Poisson kernel due to Bogdan [3]. Set $\kappa=1 / 2\left(\sqrt{1+K^{2}}\right)$, where $K$ is the Lipschitz constant of the domain, seen locally as the graph of a function from $\mathbb{R}^{N-1}$ into $\mathbb{R}$. Let $x_{0} \in \Omega$ and set $\phi(x):=G\left(x, x_{0}\right)$. Then there exists $c_{1}>0$ such that for any $y \in \partial \Omega$ and $x \in \Omega$ satisfying $|x-y| \leq r_{0}$, there holds

$$
c_{1}^{-1} \frac{\phi(x)}{\phi^{2}(\xi)}|x-y|^{2-N} \leq K(x, y) \leq c_{1} \frac{\phi(x)}{\phi^{2}(\xi)}|x-y|^{2-N},
$$

for any $\xi$ such that $B_{\kappa|x-y|}(\xi) \subset \Omega \cap B_{|x-y|}(y)$. This implies

$$
c_{2}^{-1} \frac{\phi^{q+1}(x)}{\phi^{2 q}(\xi)}|x-y|^{(2-N) q} \leq K^{q}(x, y) \rho(x) \leq c_{2} \frac{\phi^{q+1}(x)}{\phi^{2 q}(\xi)}|x-y|^{(2-N) q}
$$

for some $c_{2}$ since $\phi$ and $\rho$ are comparable in $B_{r_{0}}(y)$, uniformly with respect to $y$ (provided we have chosen $r_{0} \leq \operatorname{dist}\left(x_{0}, \partial \Omega\right) / 2$. Let $C_{s, y}$ be a smooth cone with vertex at $y$ and opening $S:=C_{s, y} \cap \partial B_{1}(y)$, such that $\bar{C}_{s, y} \cap \partial B_{r_{0}}(y) \subset \Omega$. We can impose to the point $\xi$ in inequality (5.57) to be such that $\xi /|\xi|:=\Xi_{0} \in S$, or, equivalently, such that $|\xi-y| \leq \gamma$ dist $(\xi, \partial \Omega)$ for some $\gamma>1$ independent of $\xi$, $|x-y|$ and $y$. Then, by Carleson estimate [2, Lemma 2.4] and Harnack inequality, there exists $c_{5}$ independent of $y$ such that there holds

$$
\frac{\phi(\xi)}{\phi(x)} \geq c_{3}
$$

for all $x \in \Omega \cap B_{r_{0}}(y)$ and all $\xi$ as above. Consequently, (5.58) yields to

$$
K^{q}(x, y) \rho(x) \leq c_{4} \phi^{1-q}(\xi)|x-y|^{(2-N) q} .
$$

There exists a separable harmonic function $v$ in $C_{s, y}$ under the form

$$
v(z)=|z-y|^{\alpha_{S}+2-N} \phi_{S}((z-y) /|z-y|)
$$

where $\phi_{S}$ is the first eigenfunction of $-\Delta^{\prime}$ in $W_{0}^{1,2}(S)$ normalized by $\max \phi_{S}=$ $1, \lambda_{S}$ the corresponding eigenvalue and $\alpha_{S}$ is given by (5.10). By the maximum principle,

$$
v(z) \leq c_{5} \phi(z) \quad \forall z \in C_{S, y} \cap B_{r_{0}}(y) .
$$


Therefore there exists $c_{6}>0$ such that

$$
\phi(\xi) \geq c_{6}|\xi-y|^{\alpha}{ }^{+2-N} .
$$

Because $|x-y| \geq|\xi-y| \geq \kappa|x-y| / 2$, from the choice of $\xi$, it follows

$$
K^{q}(x, y) \rho(x) \leq \frac{c_{7}}{|x-y|^{(q-1) \alpha_{S}+N-2}} \quad \forall x \in \Omega \cap B_{r_{0}}(y) .
$$

Clearly, if we choose $q$ such that $1<q<q_{S_{y}^{I}}:=1+2 / \alpha_{S_{y}^{I}}$, then $q<1+2 / \alpha_{s_{r, y}^{I}}$ for some $r$ small enough and we can take $C_{S, y}=C_{y, r}^{I}$. Thus (5.55) follows.

We turn to the proof of (5.56). To simplify the notation we assume that $q<$ $q_{\partial \Omega}^{*}$. The argument is the same in the case $q<q_{E}^{*}$.

If we assume $q<\lim _{r \rightarrow 0} \inf \left\{q_{S_{z, r}^{I}}: z \in \partial \Omega\right\}$, then for $\epsilon>0$ small enough, there exists $r_{\epsilon}>0$ such that

$$
0<r \leq r_{\epsilon} \Longrightarrow 1<q<\inf \left\{q_{S_{z, r}^{I}}: z \in \partial \Omega\right\}-\epsilon \quad \forall 0<r \leq r_{\epsilon} .
$$

Notice that the shape of the cone may vary, but, since $\partial \Omega$ is Lipschitz there exists a fixed relatively open subdomain $S^{*} \subset \partial B_{1}$ such that for any $y \in \partial \Omega$, there exists an isometry $\mathcal{R}_{y}$ of $\mathbb{R}^{N}$ with the property that $\mathcal{R}_{y}\left(\bar{S}^{*}\right) \subset S_{y, r}^{I}$ for all $0<r \leq r_{\epsilon}$. Here we use the fact that $r \mapsto S_{y, r}^{I}$ is increasing when $r$ decreases. If we take $\xi$ such that $\xi /|\xi|=\Xi_{0} \in \mathcal{R}_{y}\left(S^{*}\right)$, then the constants in Bogdan estimate (5.57) and Carleson inequality (5.59) are independent of $y \in \partial \Omega$ if we replace $r_{0}$ by $\inf \left\{r_{\epsilon}, r_{0}\right\}$. Hereafter we shall assume that $r_{\epsilon} \leq r_{0}$. Set

$$
v_{S}(t)=|t-y|^{\alpha_{S}+2-N} \phi_{S}((t-y) /|t-y|)
$$

with $S=S_{y, r_{\epsilon}}^{I}$. Then $v_{S}$ is well defined in the cone $C_{S, y}$ with vertex $y$ and opening $S$. Let

$$
\Sigma_{c r_{\epsilon}}:=\left\{t \in \Omega: \operatorname{dist}(t, \partial \Omega)=c r_{\epsilon}\right\} .
$$

Because $\partial \Omega$ is Lipschitz, we can choose $0<c<1$ such that $C_{S, y} \cap \Sigma_{c r_{\epsilon}} \subset B_{r_{\epsilon}}(z)$. Then we can compare $v_{S}$ and $\phi$ on the set $\Sigma_{c r_{\epsilon}}$. It follows by maximum principle that estimate (5.61) is still valid with a constant may depend on $r_{\epsilon}$, but not on $y$. Because

$$
\min _{\mathcal{R}_{y}\left(S^{*}\right)} \phi_{S_{y, r_{\epsilon}}^{I}} \geq c_{8}
$$

where $c_{8}$ is independent of $y,(5.62)$ holds under the form

$$
\phi(\xi) \geq c_{6}|\xi-y|^{\alpha} s_{y, r_{\epsilon}}^{+2-N},
$$

where, we recall it, $\xi$ satisfies $\xi /|\xi| \in \mathcal{R}_{y}\left(S^{*}\right)$, and is associated to any $x \in B_{r_{\epsilon}}(y) \cap$ $\Omega$ by the property that $B_{\kappa|x-y|}(\xi) \subset B_{|x-y|}(y) \cap \Omega$, and thus $|x-y| \geq|\xi-y| \geq$ $\kappa|x-y| / 2$. Then (5.63) holds uniformly with respect to $y$, with $r_{0}$ replaced by $r_{\epsilon}$. This implies (5.56). 
The next proposition partially complements Theorem 5.16.

Proposition 5.17. Let $y \in \partial \Omega$ and $q>q_{s_{y}^{o}}$. Then any solution of (5.1) in $\Omega$ which vanishes on $\partial \Omega \backslash\{0\}$ is identically 0 .

Remark 5.18. This proposition implies that, if $q>q_{S_{y}^{O}}$,

$$
\int_{\Omega} K^{q}(x, y) \rho(x) d x=\infty .
$$

Otherwise $\delta_{y}$ would be admissible.

Proof. We consider a local outer smooth cone with vertex at $y, C_{2}$, such that $\bar{\Omega} \cap$ $B_{r_{0}}(y) \backslash\{0\} \subset C_{2} \cap B_{r_{0}}(y):=C_{2, r_{0}}$. We denote by $S^{*}=C_{2} \cap \partial B_{1}(y)$ its opening. For $\epsilon>0$ small enough, we consider the doubly truncated cone $C_{2, r_{0}}^{\epsilon}=\cap C_{2, r_{0}} \backslash$ $\left.B_{\epsilon}(y)\right\}$ and the solution $v:=v_{\epsilon}$ to

$$
\begin{cases}-\Delta v+v^{q}=0 & \text { in } C_{2, r_{0}}^{\epsilon} \\ v=\infty & \text { on } \partial B_{\epsilon}(y) \cap C_{2} \\ v=\infty & \text { on } \partial B_{r_{0}}(y) \cap C_{2} \\ v=0 & \text { on } \partial C_{2} \cap B_{r_{0}}(y) \backslash \bar{B}_{\epsilon}(y),\end{cases}
$$

where $q \geq q_{S^{*}}:=1+2 / \alpha_{S^{*}}$, and $\alpha_{S^{*}}$ is expressed by (5.10) with $S$ replaced by $S^{*}$. Then $v_{\epsilon}$ dominates in $C_{2, r_{0}}^{\epsilon} \cap \Omega$ any positive solution $u$ of (5.1) in $\Omega$ which vanishes on $\partial \Omega \backslash\{0\}$. Letting $\epsilon \rightarrow 0, v_{\epsilon}$ converges to $v_{0}$ which satisfies

$$
\begin{cases}-\Delta v+v^{q}=0 & \text { in } C_{2, r_{0}} \\ v=\infty & \text { on } \partial B_{r_{0}} \cap C_{2} \\ v=0 & \text { on } \partial C_{2} \cap B_{r_{0}}(y) .\end{cases}
$$

Furthermore $u \leq v_{0}$ in $B_{r_{0}} \cap \Omega$. Because $q_{S^{*}}$ is the critical exponent in $C_{2}$, the singularity at 0 is removable, which implies that $v(x) \rightarrow 0$ when $x \rightarrow 0$ in $C_{2}$. Thus $u_{+}(x) \rightarrow 0$ when $x \rightarrow 0$ in $\Omega$. Thus $u_{+}=0$. But we can take any cone with vertex $y$ containing $\Omega$ locally in $B_{r}(y)$ for $r>0$. This implies that for any $q>q_{S_{y}^{O}}$, any solution of (5.1) which vanishes on $\partial \Omega \backslash\{0\}$ is non-positive. In the same way it is non-negative.

Definition 5.19. If $y \in \partial \Omega$ we say that an exponent $q \geq 1$ is:

(i) Admissible at $y$ if

$$
\|K(., y)\|_{L_{\rho}^{q}(\Omega)}<\infty
$$

and we set

$$
q_{1, y}=\sup \{q>1: q \text { admissible at } y\} .
$$


(ii) Acceptable at $y$ if there exists a solution of (5.1) with boundary trace $\delta_{y}$, and we set

$$
q_{2, y}=\sup \{q>1: q \text { acceptable at } y\} .
$$

(iii) Super-critical at $y$ if any solution of (5.1) which is continuous in $\Omega \backslash\{0\}$ and vanishes on $\partial \Omega \backslash\{0\}$ is identically zero, and we set

$$
q_{3, y}=\inf \{q>1: q \text { super-critical at } y\} .
$$

Proposition 5.20. Assume $\Omega$ is a bounded Lipschitz domain and $y \in \partial \Omega$. Then

$$
q_{S_{y}^{I}} \leq q_{1, y} \leq q_{2, y} \leq q_{3, y} \leq q_{S_{y}^{O}}
$$

If $1<q<q_{2, y}$ then, for any real a there exists exactly one solution of (5.1) with boundary trace $\gamma \delta_{y}$.

Proof. It follows from Theorem 5.16 that $q_{S_{y}^{I}} \leq q_{1, y}$ and from Proposition 5.17 that $q_{3, y} \leq q_{S_{y}}$. It is clear from the definition and Theorem 3.10 that $q_{1, y} \leq q_{2, y} \leq q_{3, y}$. Thus (5.68) holds.

Now assume that $q<q_{2, y}$ so that there exists a solution $u$ with boundary trace $\delta_{y}$. By the maximum principle $u>0$ in $\Omega$. If $a \in(0,1)$ then $a u$ is a subsolution of (5.1) with boundary trace $a \delta_{y}$ and $a u<u$. Therefore by Corollary 4.4 II, the smallest solution dominating $a u$ has boundary trace $a \delta_{y}$. If $a>1$ then $a u$ is a supersolution and the same conclusion follows from Corollary 4.4 I. If $v_{a}$ is the (unique) solution of (5.1) with boundary trace $a \delta_{y}$ then $-v$ is the (unique) solution with boundary trace $-a \delta_{y}$.

Theorem 5.21. Assume $y \in \partial \Omega$ is such that $S_{y}^{O}=S_{y}^{I}=S$, let $\lambda_{S}$ be the first eigenvalue of $-\Delta^{\prime}$ in $W_{0}^{1,2}(S)$ and denote

$$
q_{c, y}:=1+2 / \alpha_{S}
$$

with $\alpha_{S}$ as in (5.10). Then $q_{1, y}=q_{2, y}=q_{3, y}=q_{c, y}$ and

(i) if $1<q<q_{c, y}$ then $\delta_{y}$ is admissible;

(ii) if $q>q_{c, y}$ then the only solution of (5.1) in $\Omega$ vanishing on $\partial \Omega \backslash\{y\}$ is the trivial solution;

(iii) if $q=q_{c, y}$ and $u$ is a solution of (5.1) in $\Omega$ vanishing on $\partial \Omega \backslash\{y\}$ then

$$
u=o(1)|x-y|^{-\frac{2}{q-1}} \text { as } x \rightarrow y \text { in } \Omega \text {. }
$$

Remark 5.22. We know that, in the conical case, the conclusion of statement (ii) holds for $q=q_{c, y}$ as well. Consequently, in a polyhedral domain $\Omega$, an isolated singularity at a point $y \in \partial \Omega$ is removable if $q \geq q_{c}(y)$. We do not know if this holds in general Lipschitz domains. 
Proof. The above assertion, except for statement (iii), is an immediate consequence of Proposition 5.20, Definition 5.12 and the remark following that definition.

It remains to prove (iii). We may assume that $u>0$. Otherwise we observe that $|u|$ is a subsolution of (5.1) and by Theorem 4.3(ii) there exists a solution $v$ dominating it. It is easy to verify that the smallest solution dominating $|u|$ vanishes on $\partial \Omega \backslash\{y\}$.

For any $r>0$ let $u_{r}$ be the extension of $u$ by zero to $D_{r}:=C_{S_{r}} \cap B_{r}(y)$. Thus $u_{r}$ is a subsolution in $D_{r}, u_{r} \in C\left(\bar{D}_{r} \backslash\{y\}\right)$ and $u_{r}=0$ on $\left(\partial C_{S_{r}} \cap B_{r}(y)\right) \backslash\{y\}$. The smallest solution above it, say $\tilde{u}_{r}$ is in $C\left(\bar{D}_{r} \backslash\{y\}\right)$ and $\tilde{u}_{r}=0$ on $\left(\partial C_{S_{r}} \cap B_{r}(y)\right) \backslash$ $\{y\}$. By a standard argument this implies that there exists a positive solution $\tilde{v}_{r}$ in $D_{r}$ such that $\tilde{v}_{r}$ vanishes on $\partial D_{r} \backslash\{y\}$ and

$$
u_{r} \leq 2 \tilde{v}_{r} \text { in } D_{r} .
$$

We extend this solution by zero to the entire cone $C_{S_{r}^{O}}$, obtaining a subsolution $\tilde{w}_{r}$ and finally (again by Theorem 4.3(ii)) a solution $w_{r}$ in $C_{S_{r}^{O}}$ which vanishes on $\partial C_{S_{r}} \backslash\{y\}$ and satisfies

$$
u_{r} \leq 2 w_{r} \text { in } D_{r} .
$$

Observe that $q_{S_{r}^{O}} \downarrow q_{c, y}$ as $r \downarrow 0$. If $q_{c, y}=q_{S_{r}}$ for some $r>0$ then the existence of a solution $w_{r}$ as above is impossible. Therefore we conclude that $q_{c, y}<q_{S_{r}}$ and therefore, by Theorem 5.6, there exists a solution $v_{\infty, r}$ in $C_{S_{r}^{O}}$ such that

$$
v_{\infty, r}(x)=|x-y|^{-\frac{2}{q-1}} \omega_{S_{r}}((x-y) /|x-y|) \quad \forall x \in C_{S_{r}^{O}} .
$$

This solution is the maximal solution in $C_{S_{r}^{O}}$ so that

$$
w_{r} \leq v_{\infty, r} \text { in } D_{r}
$$

But, since $q=q_{S} O$, it follows that $\omega_{S_{r}^{O}} \rightarrow 0$ as $r \rightarrow 0$. This implies (5.70).

The next result provides an important ingredient in the study of general boundary value problems in Lipschitz domains.

Theorem 5.23. Assume that $q>1, \Omega$ is a bounded Lipschitz domain and $u \in$ $\mathcal{U}_{+}(\Omega)$. If $y \in \mathcal{S}(u)$ and $q<q_{y}^{*}$ then, for every $k>0$, the measure $k \delta_{y}$ is admissible and

$$
u \geq u_{k \delta_{y}} \quad \forall k \geq 0 .
$$

Remark 5.24. Here $q_{y}^{*}=q_{\{y\}}^{*}$ is defined as in part $\mathrm{E}$ of the introduction. If $q>q_{y}^{*}$, (5.71) need not hold. For instance, consider the cone $C_{S}$ with vertex at the origin, such that $S \subset S^{N-1}$ is a smooth domain and $S^{N-1} \backslash S$ is contained in an open half space. Then $q_{c, 0}>(N+1) /(N-1)$ while $q_{c, x}=(N+1) /(N-1)$ for any $x \neq 0$ on the boundary of the cone. Thus $q^{*}(0)<q_{c, 0}$. Suppose that $q \in\left(q_{0}^{*}, q_{c, 0}\right)$. Let $F$ be a closed subset of $\partial C_{S}$ such that $0 \in F$ but 0 is a $C_{2 / q, q^{\prime}}$-thin point of $F$. Let $u$ be the maximal solution in $C_{S}$ vanishing on $\partial C_{S} \backslash F$. Then $0 \in \mathcal{S}(u)$ but (5.71) does not hold for any $k>0$. 
Proof. Up to an isometry of $\mathbb{R}^{N}$, we can assume that $y=0$ and represent $\partial \Omega$ near 0 as the graph of a Lipschitz function. This can be done in the following way: we define the cylinder $C_{R}^{\prime}:=\left\{x=\left(x^{\prime}, x_{N}\right): x^{\prime} \in B_{R}^{\prime}\right\}$ where $B_{R}^{\prime}$ is the $(N-1)$-ball with radius $R$. We denote, for some $R>0$ and $0<\sigma<R$,

$$
\partial \Omega \cap C_{R}^{\prime}=\left\{x=\left(x^{\prime}, \eta\left(x^{\prime}\right)\right): x^{\prime} \in B_{R}^{\prime}\right\},
$$

and

$$
\Sigma_{\delta, \sigma}=\left\{x=\left(x^{\prime}, \eta\left(x^{\prime}\right)+\delta\right): x^{\prime} \in B_{\sigma}^{\prime}\right\},
$$

and assume that, if $0<\delta \leq R$,

$$
\Omega_{\delta}^{R}=\left\{x=\left(x^{\prime}, x_{N}\right): x^{\prime} \in B_{R}^{\prime}, \eta\left(x^{\prime}\right)<x_{N}<\eta\left(x^{\prime}\right)+R\right\} \subset \Omega .
$$

We can also assume that $\eta(0)=0$. Although the two harmonic measures in $\Omega$ and $\partial \Omega \cap C_{R}^{\prime}$ differ, it follow by Dahlberg's result that there exists a constant $c>0$ such that, if $\delta<\delta_{0} \leq R / 2$,

$$
c^{-1} \omega_{\Omega}^{x_{0}}(E) \leq \omega_{\Omega_{\delta}^{R}}^{x_{0}}\left(E+\epsilon \mathbf{e}_{N}\right) \leq c \omega_{\Omega}^{x_{0}}(E),
$$

for any Borel set $E \subset \partial \Omega \cap C_{\delta}^{\prime}$. Therefore, if we set

$$
M_{\epsilon, \sigma}=\int_{\Sigma_{\epsilon, \sigma}} u(x) d \omega^{x_{0}},(x),
$$

it follows that $\lim _{\epsilon \rightarrow 0} M_{\epsilon, \sigma}=\infty$ since $0 \in \mathcal{S}(u)$. We can suppose that $\sigma$ is small enough so that there exists $\hat{q} \in\left(q, q_{y}^{*}\right)$ and $M>0$ such that, for any $p \in[1, \hat{q}]$

$$
\int_{\Omega} K^{p}(x, z) \rho(x) d x \leq M \quad \forall z \in \partial \Omega \cap B_{\sigma} .
$$

For fixed $k$ there exists $\epsilon=\epsilon(\delta)>0$ such that $M_{\epsilon, \sigma}=k$. There exists a uniform Lipschitz exhaustion $\left\{\Omega_{\epsilon}\right\}$ of $\Omega$ with the following properties:

(i) $\Omega_{\epsilon} \cap C_{R}^{\prime} \cap\left\{x=\left(x^{\prime}, x_{N}\right): a<x_{N}<b\right\}=\Sigma_{\epsilon, R}$, for some fixed $a$ and $b$.

(ii) The $\Omega_{\epsilon}$ and $\Omega$ have the same Lipschitz character $L$.

It follows that the Poisson kernel $K^{\Omega_{\epsilon}}$ in $\Omega_{\epsilon}$ respectively endows the same properties (5.72) as $K$ except $\Omega$ has to be replaced by $\Omega_{\epsilon}, \rho$ by $\rho_{\epsilon}:=\operatorname{dist}\left(., \partial \Omega_{\epsilon}\right.$ and $z$ has to belong to $\partial \Omega_{\epsilon} \cap B_{\sigma}$. Next, we consider the solution $v=v_{\epsilon(\sigma))}$ of

$$
\begin{cases}-\Delta v+v^{q}=0 & \text { in } \Omega_{\epsilon} \\ v=u \chi_{\Sigma_{\epsilon, \sigma}} & \text { in } \partial \Omega_{\epsilon} .\end{cases}
$$

By the maximum principle, $u \geq v$ in $\Omega_{\epsilon}$. Furthermore $v \leq \mathbb{K}^{\Omega_{\epsilon}}\left[u \chi_{\Sigma_{\epsilon, \sigma}}\right]$. Let $\hat{q}=\left(q+\tilde{q}_{\sigma}\right) / 2$ and $\omega \subset \Omega$ be a Borel subset. By convexity

$$
\int_{\omega}\left(\mathbb{K}^{\Omega_{\epsilon}}\left[u \chi_{\Sigma_{\epsilon, \sigma}}\right]\right)^{\hat{q}} \rho(x) d x \leq M M_{\epsilon, \sigma} .
$$


Thus, by Hölder's inequality

$$
\int_{\omega}\left(\mathbb{K}^{\Omega_{\epsilon}}\left[u \chi_{\Sigma_{\epsilon, \sigma}}\right]\right)^{q} \rho(x) d x \leq\left(\int_{\omega} \rho(x) d x\right)^{1-q / \hat{q}}\left(M M_{\epsilon, \sigma}\right)^{q / \hat{q}} .
$$

By standard a priori estimates, $v_{\epsilon(\sigma)} \rightarrow v_{0}$ (up to a subsequence) a.e. in $\Omega$, thus $v_{\epsilon(\sigma)}^{q} \rightarrow v_{0}^{q}$. By Vitali's theorem and the uniform integrability of the $\left\{v_{\epsilon(\sigma)}\right\}$, $v_{\epsilon(\sigma)} \rightarrow v_{0}$ in $L_{\rho}^{q}(\Omega)$. Because

$$
v_{\epsilon(\sigma)}+\mathbb{G}^{\Omega_{\epsilon}}\left[v_{\epsilon(\sigma)}^{q}\right]=\mathbb{K}^{\Omega_{\epsilon}}\left[u \chi_{\Sigma_{\epsilon, \sigma}}\right]
$$

where $\mathbb{G}^{\Omega_{\epsilon}}$ is the Green operator in $\Omega_{\epsilon}$, and

$$
\mathbb{K}^{\Omega_{\epsilon}}\left[u \chi_{\Sigma_{\epsilon, \sigma}}\right] \rightarrow M_{\epsilon, \sigma} K(., y)=k K(., y)
$$

as $\sigma \rightarrow 0$, it follows that $u \geq v_{0}$, and $v_{0}$ satisfies

$$
v_{0}+\mathbb{G}^{\Omega}\left[v_{0}^{q}\right]=k K(., y) .
$$

Then $v_{0}=u_{k \delta_{y}}$, which ends the proof.

Corollary 5.25. Let $\left\{y_{j}\right\}_{j=1}^{n} \subset \partial \Omega$ be a set of points such that

$$
q<\inf \left\{q_{y_{j}}^{*}: j=1, \ldots, n\right\} .
$$

Then, for any set of positive numbers $k_{1}, \cdots, k_{n}$, there exists a unique solution $u_{\mu}$ of (5.1) in $\Omega$ with boundary trace $\mu=\sum_{j=1}^{n} k_{j} \delta_{y_{j}}$.

$$
\text { If } u \in \mathcal{U}_{+}(\Omega) \text { and }\left\{y_{j}\right\}_{j=1}^{n} \subset \mathcal{S}(u) \text { then } u \geq u_{\mu} \text {. }
$$

Proof. From Theorem 5.23, $u \geq u_{k_{j} \delta_{y_{j}}}$ for any $j=1, \ldots, n$. Thus $u \geq \tilde{u}_{\{k\}}=$ $\max \left(u_{k_{j} \delta_{y_{j}}}\right)$, which is a subsolution with boundary trace $\sum_{j} k_{j} \delta_{y_{j}}$. But $\tilde{v}_{\{k\}}$, the solution with boundary trace $\sum_{j} k_{j} \delta_{y_{j}}$ is the smallest solution above $\tilde{u}_{\{k\}}$. Therefore the conclusion of the corollary holds.

As a consequence one obtains:

Theorem 5.26. Let $E \subset \partial \Omega$ be a closed set and assume that $q<q_{E}^{*}$. Then, for every $\mu \in \mathfrak{M}(\Omega)$ such that $\operatorname{supp} \mu \subset E$ there exists a (unique) solution $u_{\mu}$ of (5.1) in $\Omega$ with boundary trace $\mu$.

If $\left\{\mu_{n}\right\}$ is a sequence in $\mathfrak{M}(\Omega)$ such that supp $\mu_{n} \subset E$ and $\mu_{n} \rightarrow \mu$ weak* then $u_{\mu_{n}} \rightarrow u_{\mu}$ locally uniformly in $\Omega$. $\mathcal{S}(u)$,

If $u \in \mathcal{U}_{+}(\Omega)$ and $q<q_{\mathcal{S}(u)}^{*}$ then, for every $\mu \in \mathfrak{M}(\Omega)$ such that $\operatorname{supp} \mu \subset$

$$
u_{\mu} \leq u
$$


Proof. Without loss of generality we assume that $\mu \geq$ ). Let $\left\{\mu_{n}\right\}$ be a sequence of measures on $\partial \Omega$ of the form

$$
\mu_{n}=\sum_{j=1}^{k_{n}} a_{j, n} \delta_{y_{j, n}}
$$

where $y_{j, n} \in E, a_{j, n}>0$ and $\sum_{j=1}^{k_{n}} a_{j, n}=\|\mu\|$, such that $\mu_{n} \rightarrow \mu$ weakly*. Passing to a subsequence if necessary, $u_{\mu_{n}} \rightarrow v$ locally uniformly in $\Omega$. In order to prove the first assertion it remains to show that $v=u_{\mu}$.

If $0<r$ is sufficiently small, there exists $\hat{q}_{r} \in\left(q, q_{E}^{*}\right)$ and $M_{r}>0$ such that, for any $p \in\left[1, \hat{q}_{r}\right]$ and every $z \in \partial \Omega$ such that dist $(z, E)<r$, estimate $(5.72)$ holds. It follows that the family of functions

$$
\{K(\cdot, z): z \in \partial \Omega, \operatorname{dist}(z, E)<r\}
$$

is uniformly integrable in $L_{\rho}^{q}(\Omega)$ and consequently the family

$$
\left\{\mathbb{K}[v] ; \nu \in \mathfrak{M}(\partial \Omega),\|v\|_{\mathfrak{M}} \leq 1, \operatorname{supp} v \subset\{z \in \partial \Omega: \operatorname{dist}(z, E)<r\}\right\}
$$

is uniformly integrable in $L_{\rho}^{q}(\Omega)$. By a standard argument (using Vitali's convergence theorem) this implies that $v=u_{\mu}$. This proves the first two assertions of the theorem.

The last assertion is an immediate consequence of the above together with Corollary 5.25. Indeed, if $E=\mathcal{S}(u)$ then, by Corollary 5.25, $u \geq u_{\mu_{n}}$. Therefore $u \geq u_{\mu}$.

Proposition 5.27. Let $y \in \partial \Omega$ and $1<q<q_{S_{y}^{I}}$. Then there exists a maximal solution $u:=U_{y}$ of $(5.1)$ such that $\operatorname{tr}\left(U_{y}\right)=(\{y\}, 0)$. It satisfies

$$
\liminf _{\substack{x \rightarrow y \\ x-y \\|x-y|}}|x-y|^{2 /(q-1)} U_{y}(x) \geq \omega_{S_{y}^{I}}(\sigma),
$$

uniformly on any compact subset of $S_{y}^{I}$, where $\omega_{S_{y}^{I}}$ is the unique positive solution of

$$
\begin{cases}-\Delta^{\prime} \omega-\lambda_{N, q} \omega+|\omega|^{q-1} \omega=0 & \text { in } S_{y}^{I} \\ \omega=0 & \text { on } \partial S_{y}^{I},\end{cases}
$$

normalized by $\omega\left(\sigma_{0}\right)=1$ for some fixed $\sigma_{0} \in S_{y}^{I}$.

For $r>0$ small enough, we denote by $\omega_{S_{y, r}^{O}}$ the unique positive solution of

$$
\begin{cases}-\Delta^{\prime} \omega-\lambda_{N, q} \omega+|\omega|^{q-1} \omega=0 & \text { in } S_{y, r}^{O} \\ \omega=0 & \text { on } \partial S_{y, r}^{O}\end{cases}
$$


normalized in the same way. Then

$$
\limsup _{\substack{x \rightarrow y \\ x-y \\|x-y|}}|x-y|^{2 /(q-1)} U_{y}(x) \leq \omega_{S_{y, r}^{O}}(\sigma) .
$$

Finally, if $S_{y}^{O}=S_{y}^{I}=S$, then

$$
\lim _{\substack{x \rightarrow y \\ x-y \\|x-y|}}|x-y|^{2 /(q-1)} U_{y}(x)=\omega_{S}(\sigma) .
$$

Proof. We recall that $C_{y, r}^{I}$ (respectively $C_{y, r}^{O}$ ) is an $r$-inner cone (respectively $r$ outer cone) at $y$ with opening $S_{y, r}^{I} \subset \partial B_{1}(y)$ (respectively $S_{y, r}^{O} \subset \partial B_{1}(y)$ ). This is well defined for an $r>0$ small enough so that $q<q_{S_{y, r}^{I}}$. We denote by $\omega_{s_{y, r}^{I}}$ the unique positive solution of

$$
\begin{cases}-\Delta^{\prime} \omega-\lambda_{N, q} \omega+|\omega|^{q-1} \omega=0 & \text { in } S_{y, r}^{I} \\ \omega=0 & \text { on } \partial S_{y, r}^{I} .\end{cases}
$$

We construct $U_{y} \in \mathcal{U}_{+}(\Omega)$, vanishing on $\partial \Omega \backslash\{y\}$ in the following way. For $0<$ $\epsilon<r$, we denote by $v:=U_{y, \epsilon}$ the solution of

$$
\begin{cases}-\Delta v+\left|v^{q-1}\right| v=0 & \text { in } \Omega \backslash \bar{B}_{\epsilon}(y) \\ v=0 & \text { in } \partial \Omega \backslash \bar{B}_{\epsilon}(y) \\ v=\infty & \text { in } \Omega \cap \partial B_{\epsilon}(y) .\end{cases}
$$

Let $v:=V_{\epsilon}^{I}$ (respectively $v:=V_{\epsilon}^{O}$ ) be the solution of

$$
\left\{\begin{array}{lll}
-\Delta v+\left|v^{q-1}\right| v=0 & \text { in } C_{S_{y, r}^{I}} \backslash \bar{B}_{\epsilon}(y) & \text { (respectively } \left.C_{S_{y, r}^{O}} \backslash \bar{B}_{\epsilon}(y)\right) \\
v=0 & \text { in } \partial C_{S_{y, r}^{I}} \backslash \bar{B}_{\epsilon}(y) & \text { (respectively } \left.\partial C_{S_{y, r}^{O}} \backslash \bar{B}_{\epsilon}(y)\right) \\
v=\infty & \text { in } \left.C_{S_{y, r}^{I}} \cap \partial B_{\epsilon}(y) \text { (respectively } C_{S_{y, r}^{O}} \cap \partial B_{\epsilon}(y)\right) .
\end{array}\right.
$$

Then there exist $m>0$ depending on $r$, but not on $\epsilon$, such that

$$
V_{\epsilon}^{I}(x)-m \leq U_{y, \epsilon}(x) \leq V_{\epsilon}^{O}(x)+m
$$

for all $x \in C_{y, r}^{I} \backslash\left\{B_{\epsilon}(y)\right\}$ for the left-hand side inequality, and $x \in \partial \Omega \cap B_{r}(y) \backslash$ $\left\{B_{\epsilon}(y)\right\}$ for the right-hand side one. When $\epsilon \rightarrow 0, V_{\epsilon}^{I}$ converges to the explicit separable solution $x \mapsto|x-y|^{-2 /(q-1)} \omega_{s_{y, r}^{I}}$ in $C_{s_{y, r}^{I}}$ (the positive cone with vertex generated by $S_{y, r}^{I}$ ). Similarly $V_{\epsilon}^{O}$ converges to the explicit separable solution $x \mapsto$ 
$|x-y|^{-2 /(q-1)} \omega_{S_{y, r}^{O}}$ in $C_{S_{y, r}^{O}}$. Furthermore $\epsilon<\epsilon^{\prime} \Longrightarrow U_{y, \epsilon} \leq U_{y, \epsilon^{\prime}}$. If $U_{y}=$ $\lim _{\epsilon \rightarrow 0}\left\{U_{y, \epsilon}\right\}$, there holds

$$
\begin{aligned}
& |x-y|^{-2 /(q-1)} \omega_{S_{y, r}^{I}}\left(\frac{x-y}{|x-y|}\right)-m \leq U_{y}(x) \\
& \leq|x-y|^{-2 /(q-1)} \omega_{S_{y, r}^{I}}\left(\frac{x-y}{|x-y|}\right)+m .
\end{aligned}
$$

These inequalities imply

$$
\liminf _{\substack{x \rightarrow y \\ x-y \\|x-y|}}|x-y|^{2 /(q-1)} U_{y}(x) \geq \omega_{S_{y, r}^{I}}(\sigma) .
$$

Inequality (5.79) is obtained in a similar way. Since $\lim _{r \rightarrow 0} \omega_{S_{y, r}^{I}}=\omega_{S_{y}^{I}}$ uniformly in compact subsets of $S_{y}^{I}$ we also obtain (5.76). If $S_{y}^{O}=S_{y}^{I}=S$, then $\omega_{S_{y}^{I}}=$ $\omega_{S_{y}^{O}}=\omega_{S}$, thus (5.80) holds.

Remark 5.28. Because $U_{y}$ is the maximal solution which vanishes on $\partial \Omega \backslash\{y\}$, the function $u_{\infty \delta_{y}}=\lim _{k \rightarrow \infty} u_{k \delta_{y}}$ also satisfies inequality (5.79). We conjecture that $u_{\infty \delta_{y}}$ always satisfies estimate (5.76). This is true if the outer and inner cone at $y$ are the same. In fact in that case we obtain a much stronger result:

Theorem 5.29. Assume $y \in \partial \Omega$ is such that $S_{y}^{O}=S_{y}^{I}=S$ and $q<q_{c, y}$. Then $U_{y}=u_{\infty \delta_{y}}$.

Proof. Without loss of generality we can assume that $y=0$ and will denote $B_{r}=$ $B_{r}(0)$ for $r>0$. Let $C_{r}^{I}$ (respectively $C_{r}^{O}$ ) be a cone with vertex 0 , such that $\overline{C_{r}^{I} \cap B_{r}} \backslash\{0\} \subset \Omega$ (respectively $\Omega \cap B_{r} \subset C_{r}^{O}$ ). We recall that the characteristic exponents $\alpha_{S_{0}^{I}}$ and $\alpha_{S_{0}^{O}}$ are defined according to Definition 5.10 and Definition 5.12. Since

$$
\alpha_{S_{0}^{I}}=\lim _{r \rightarrow 0} \alpha_{S_{0, r}^{I}}=\lim _{r \rightarrow 0} \alpha_{S_{0, r}^{O}}=\alpha_{S_{0}^{O}}<2 /(q-1),
$$

we can choose $r$ such that

$$
q \alpha_{S_{0, r}^{I}}-\alpha_{S_{0, r}^{O}}<2-(q-1)\left(\alpha_{S_{0, r}^{I}}-\alpha_{S_{0, r}^{O}}\right),
$$

and for simplicity, we set $\alpha_{s_{0, r}^{I}}=\alpha_{I}, \alpha_{s_{0, r}^{O}}=\alpha_{O}$ and

$$
\gamma_{r}=\frac{q-1}{2+\alpha_{o}-q \alpha_{I}} .
$$

Step 1 . We claim that there exists $c>0$ and $c^{*}>0$ such that, for any $m>0$

$$
u_{m \delta_{0}}(x) \geq c^{*} m|x|^{-\alpha_{O}} \quad \forall x \in B_{c m^{-\gamma r}} \cap C_{r}^{I} .
$$


Since $m K(., 0)$ is a super-solution for (5.1),

$$
u_{m \delta}(x) \geq m K(x, 0)-m^{q} \int_{\Omega} G(z, x) K^{q}(z, 0) d z .
$$

If we assume that $x \in C_{r}^{I} \cap B_{r}$, then $\operatorname{dist}(x, \partial \Omega) \geq \theta|x|$ for some $\theta>0$ since $\overline{C_{r}^{I} \cap B_{r}} \backslash\{0\} \subset \Omega$. Using Bogdan's estimate and Harnack inequality we derive

$$
K(x, 0) \geq c_{1} \frac{|x|^{2-N}}{G\left(x, x_{0}\right)},
$$

for some fixed point $x_{0}$ in $\Omega$. But the Green function in $\Omega \cap B_{r}$ is dominated by the Green function in $C_{r}^{O} \cap B_{r}$, thus $G\left(x, x_{0}\right) \leq c_{2}|x|^{\tilde{\alpha}_{O}}$ where $\tilde{\alpha}_{O}=2-N+\alpha_{O}$. This implies

$$
K(x, 0) \geq c_{3}|x|^{-\alpha_{O}} \quad \forall x \in C_{r}^{I} \cap B_{r} .
$$

Similarly (and it is a very rough estimate)

$$
K(x, 0) \leq c_{4}|x|^{-\alpha_{I}} \quad \forall x \in \Omega .
$$

Because $G(x, z) \leq c_{5}|x-z|^{2-N}$, we obtain

$$
\int_{\Omega} G(z, x) K^{q}(z, 0) d z \leq c_{6} \int_{B_{R}}|x-z|^{2-N}|z|^{-\alpha} I d z .
$$

We write

$$
\begin{aligned}
\int_{B_{R}}|x-z|^{2-N}|z|^{-q \alpha} I d z= & \int_{B_{2|x|}}|x-z|^{2-N}|z|^{-q \alpha} I d z \\
& +\int_{B_{R} \backslash B_{2|x|}|x-z|^{2-N}|z|^{-q \alpha} I z .}
\end{aligned}
$$

But

$$
\int_{B_{2|x|}}|x-z|^{2-N}|z|^{-q \alpha_{I}} d z=|x|^{2-q \alpha_{I}} \int_{B_{2}(0)}|\xi-t|^{2-N}|t|^{-q \alpha_{I}} d t
$$

where $\xi=x /|x|$ is fixed. In the same way

$$
\begin{aligned}
\int_{B_{R} \backslash B_{2|x|}}|x-z|^{2-N}|z|^{-q \alpha_{I}} d z & \leq \int_{B_{R} \backslash B_{2|x|}}|z|^{2-N-q \alpha_{I}|x|^{2-q \alpha_{I}} d z} \\
& \leq|x|^{2-q \alpha_{I}} \int_{B_{R /|x|} \backslash B_{2}}|t|^{2-N-q \alpha_{I}} d t \\
& \leq c_{7}|x|^{2-q \alpha_{I}} \int_{2}^{R /|x|} s^{1-q \alpha_{I}} d s .
\end{aligned}
$$


Thus

$$
\int_{B_{R} \backslash B_{2|x|}}|x-z|^{2-N}|z|^{-q \alpha_{I}} d z \leq \begin{cases}c_{8} & \text { if } 1-q \alpha_{I}>-1 \\ c_{8}|\ln | x|| & \text { if } 1-q \alpha_{I}=-1 \\ c_{8}|x|^{2-q \alpha_{I}} & \text { if } 1-q \alpha_{I}<-1 .\end{cases}
$$

Combining (5.87) and (5.88) yields to (5.86).

Step 2. There holds

$$
u_{\infty \delta_{0}}(x) \geq\left(|x|^{-2 / q-1)}-r^{-2 /(q-1)}\right) \omega_{S_{r}^{I}}(x /|x|) \quad \forall x \in C_{r}^{I} \cap B_{r}
$$

where $\omega_{S_{r}^{I}}$ is the unique positive solution of (5.81). For $\ell>0$, let $u_{\ell \delta_{0}}^{I}$ be the solution of

$$
\begin{cases}-\Delta u+u^{q}=0 & \text { in } C_{r}^{I} \\ u=\ell \delta_{0} & \text { on } \partial C_{r}^{I} .\end{cases}
$$

By comparing $u_{\ell \delta_{0}}^{I}$ with the Martin kernel in $C_{r}^{I}$,

$$
u_{\ell \delta_{0}}^{I}(x) \leq c_{10} \ell|x|^{-\alpha_{I}} \quad \forall x \in C_{r}^{I} .
$$

Because

$$
c_{10} \ell|x|^{-\alpha_{I}} \leq c^{*} m|x|^{-\alpha_{O}} \quad \forall x \quad \text { s.t. }|x| \geq c_{11}\left(\frac{\ell}{m}\right)^{\left(\alpha_{I}-\alpha_{O}\right)^{-1}},
$$

it follows

$$
u_{m \delta_{0}}(x) \geq u_{\ell \delta}^{I}(x) \quad \forall x \quad \text { s.t. } c_{11}\left(\frac{\ell}{m}\right)^{\left(\alpha_{I}-\alpha_{O}\right)^{-1}} \leq|x| \leq c^{*} m^{-\gamma_{r}} .
$$

Notice that (5.85) implies

$$
\left(\frac{\ell}{m}\right)^{\left(\alpha_{I}-\alpha_{0}\right)^{-1}}=o\left(m^{-\gamma_{r}}\right) \text { as } m \rightarrow \infty .
$$

Since $u_{\ell \delta_{0}}^{I}(x) \leq|x|^{-2 /(q-1)} \omega_{S_{r}^{I}}(x /|x|)$, it follows, by the maximum principle, that

$$
u_{m \delta_{0}}(x) \geq u_{\ell \delta_{0}}^{I}(x)-r^{-2 /(q-1)} \omega_{S_{r}^{I}}(x /|x|)
$$

for every $x \in C_{r}^{I} \cap B_{r}$ such that $|x| \geq c_{11}\left(\frac{\ell}{m}\right)^{\left(\alpha_{I}-\alpha_{O}\right)^{-1}}$. Letting successively $m \rightarrow \infty$ and $\ell \rightarrow \infty$ and using

$$
\lim _{\ell \rightarrow \infty} u_{\ell \delta_{0}}^{I}(x)=|x|^{-2 /(q-1)} \omega_{S_{r}^{I}}(x /|x|) \quad \forall x \in C_{r}^{I},
$$

we obtain (5.89). 
Step 3. Let $u \in \mathcal{U}_{+}(\Omega), u$ vanishing on $\partial \Omega \backslash\{0\}$. Because

$$
u(x) \leq C_{N, q}|x|^{-2 /(q-1)}
$$

and $\overline{C_{r}^{I} \cap B_{r}} \backslash\{0\} \subset \Omega$, it is a classical consequence of Harnack inequality that, for any $x$ and $x^{\prime} \in \overline{C_{r}^{I} \cap B_{r / 2}}$ such that $2^{-1}|x| \leq\left|x^{\prime}\right| \leq 2|x|, u$ satisfies

$$
c_{12}^{-1} u\left(x^{\prime}\right) \leq u(x) \leq c_{12} u\left(x^{\prime}\right),
$$

where $c_{12}>0$ depends on $N, q$ and $\min \left\{\operatorname{dist}(z, \partial \Omega) /|z|: z \in \overline{C_{r}^{I} \cap B_{r}}\right\}$.

Step 4 . There exists $c_{13}=c_{13}(q, \Omega)>0$ such that

$$
U_{0}(x) \leq c_{13} u_{\infty \delta}(x) \quad \forall x \in \Omega .
$$

Because of (5.79) and the fact that for $r>0$ and any compact subset $K \subset S_{0, r}^{I}$

$$
1 \leq \frac{\omega_{s_{0, r}^{O}}(\sigma)}{\omega_{s_{0, r}^{I}}(\sigma)} \leq M \quad \forall \sigma \in K,
$$

where $M$ depends on $K$, there exists $c_{14}>0$ such that

$$
1 \leq \frac{U_{0}(x)}{u_{\infty \delta_{0}}(x)} \leq c_{14} \quad \forall x \in B_{r} \text { s.t. } x /|x| \in K .
$$

Using Step 3, there also holds

$$
\begin{aligned}
c_{15}^{-1} & \leq \min \left\{\frac{U_{0}\left(x^{\prime}\right)}{U_{0}(x)}, \frac{u_{\infty \delta_{0}}\left(x^{\prime}\right)}{u_{\infty \delta_{0}}(x)}\right\} \\
& \leq \max \left\{\frac{U_{0}\left(x^{\prime}\right)}{U_{0}(x)}, \frac{u_{\infty \delta_{0}}\left(x^{\prime}\right)}{u_{\infty \delta_{0}}(x)}\right\} \leq c_{15} \quad \forall x, x^{\prime} \in B_{r / 2},
\end{aligned}
$$

provided $x /|x|$ and $x^{\prime} /\left|x^{\prime}\right| \in K$ and $2^{-1}|x| \leq\left|x^{\prime}\right| \leq 2|x|$. For $0<s \leq r / 2$, set $\Gamma_{s}=\Omega \cap \partial B_{s}$. There exists $n_{0} \in \mathbb{N}_{*}$ and $\kappa \in(0,1 / 4)$, independent of $s$, such that for any $x \in \Gamma_{s}$ such that $x /|x| \in K$, there exists at most $n_{0}$ points $a_{j}\left(j=1, \ldots j_{x}\right)$ such that $a_{j} \in \Gamma_{s}, a_{1} \in \partial \Omega, \kappa s \leq \operatorname{dist}\left(a_{j}, \partial \Omega\right) \leq s,\left|a_{j}-a_{j+1}\right| \leq s / 2$ for $j=1, \ldots j_{x}$ and $a_{j_{x}}=x$. Using Proposition 6.1 and the remark hereafter,

$$
c^{-1} \frac{U_{0}(z)}{U_{0}\left(a_{1}\right)} \leq \frac{u_{\infty \delta_{0}}(z)}{u_{\infty \delta_{0}}\left(a_{1}\right)} \leq c \frac{U_{0}(z)}{U_{0}\left(a_{1}\right)} \quad \forall z \in \Gamma_{s} \cap B_{a_{0}} .
$$

Combining with (5.95) we derive

$$
U_{0}(x) \leq c c_{15}^{n_{0}} u_{\infty \delta_{0}}(x) \quad \forall x \in \Gamma_{s} .
$$


Because $c c_{15}^{n_{0}} u_{\infty \delta_{0}}$ is a super-solution of (5.1) (clearly $c c_{15}^{n_{0}}>1$ ),

$$
U_{0} \leq c c_{15}^{n_{0}} u_{\infty \delta_{0}} \text { in } \Omega \backslash \bar{B}_{s} \quad \forall s \in(0, r] .
$$

Thus (5.94) follows with $c_{13}=c c_{15}^{n_{0}}$.

Step 5. End of the proof. It is based upon an idea introduced in [20]. If we assume $U_{0}>u_{\infty \delta_{0}}$, the convexity of $x \mapsto x^{q}$ implies that the function

$$
v=u_{\infty \delta_{0}}-\frac{1}{2 c_{13}}\left(U_{0}-u_{\infty \delta_{0}}\right)
$$

is a super solution such that

$$
a u_{\infty \delta_{0}} \leq v<u_{\infty \delta_{0}}
$$

where $a=\frac{1+c_{13}}{2 c_{13}}<1$. Since $a u_{\infty \delta_{0}}$ is a subsolution, it follows that there exists a solution $w$ such that

$$
a u_{\infty \delta_{0}}<w<v<u_{\infty \delta_{0}} .
$$

But this is impossible because, for any $a \in(0,1)$, the smallest solution dominating $a u_{\infty \delta_{0}}$ is $u_{\infty \delta_{0}}$.

The next result extends a theorem of Marcus and Véron [20].

Theorem 5.30. Assume that $\Omega$ is a bounded Lipschitz domain such that $S_{y}^{O}=S_{y}^{I}=$ $S_{y}$ for every $y \in \partial \Omega$. Further, assume that

$$
1<q<q_{\partial \Omega}^{*} .
$$

Then for any outer regular Borel measure $\bar{v}$ on $\partial \Omega$ there exists a unique solution $u$ of (5.1) such that $\operatorname{tr}_{\partial \Omega}(u)=\bar{v}$.

Proof. We assume $\bar{v} \sim(v, F)$ in the sense of Definition 4.9 where $F$ is a closed subset of $\partial \Omega$ and $v$ a Radon measure on $\mathcal{R}=\partial \Omega \backslash F$. We denote by $U_{F}$ the maximal solution of (5.1) defined in Lemma 4.13. Because $q<q_{\partial \Omega}^{*}$, for any $y \in F$ there exists $u_{\infty \delta_{y}}$ (and actually $u_{\infty \delta_{y}}=U_{y}$ by Theorem 5.29). Then $U_{F} \geq U_{y}$ by Lemma 4.15, thus $\mathcal{S}\left(U_{F}\right)=F^{\prime}=F$ with the notation of Definition 4.14. By Theorem 5.26, any Radon measure is q-admissible thus for any compact subset $E \subset$ $\mathcal{R}$ there exist a unique solution $u_{v \chi_{E}}$ of (5.1) with boundary trace $v \chi_{E}$. Therefore there exists a solution with boundary trace $\bar{v}$ and, by Theorem 4.16, its uniqueness is reduced to showing that $U_{F}$ is the unique solution with boundary trace $(0, F)$. Assume $u_{F}$ is any solution with trace $(0, F)$. By Theorem 5.23 and Theorem 5.29, there holds

$$
u_{F}(x) \geq u_{\infty \delta_{y}}(x)=U_{y}(x) \quad \forall y \in F, \forall x \in \Omega .
$$

Next we prove: 
Assertion. There exists $C>0$ depending on $F, \Omega$ and $q$ such that

$$
U_{F}(x) \leq C u_{F}(x) \quad \forall x \in \Omega .
$$

There exists $r_{0}>0$ and a circular cone $C_{0}$ with vertex 0 and opening $S_{0} \subset \partial B_{1}$ such that for any $y \in \partial \Omega$ there exists an isometry $T_{y}$ of $\mathbb{R}^{N}$ such that $T_{y}\left(\bar{C}_{0}\right) \cap B_{r_{0}}(y) \subset$ $\Omega \cup\{y\}$. We shall denote by $C_{1}$ a fixed sub-cone of $C_{0}$ with vertex 0 and opening $S_{1} \Subset S_{0}$. In order to simplify the geometry, we shall assume that both $C_{0}$ and $C_{0}$ are radially symmetric cones. If $x \in \Omega$ is such that dist $(x, \partial \Omega) \leq r_{0} / 2$, either

(i) there exists some $y \in \mathcal{S}$ and an isometry $T_{y}$ such that $T_{y}\left(\bar{C}_{0}\right) \cap B_{r_{0}}(y) \subset \Omega \cup\{y\}$ and $(x-y) /|x-y| \in S_{1}$,

(ii) or such a $y$ and $\mathcal{R}_{y}$ does not exist.

In the first case, it follows from Proposition 5.27 and Theorem 5.29 that

$$
u_{F}(x) \geq c_{1}|x-y|^{-2 /(q-1)} .
$$

Furthermore, the constant $c_{1}$ depends on $r, S q$ and $\Omega$, but not on $u_{F}$. By (5.5)

$$
U_{F}(x) \leq c_{2}(\operatorname{dist}(x, \partial \Omega))^{-2 /(q-1)} .
$$

Since in case (i), there holds dist $(x, \partial \Omega) \geq c_{3}|x-y|$ for some $c_{3}>1$ depending on $S_{0}$ and $S_{1}$, it follows that (5.97) holds with $c=c_{1} c_{2}^{2 /(q-1)} / c_{3}$.

In case (ii), $x$ does not belong to any cone radially symmetric cones with opening $S_{1}$ and vertex at some $y \in \mathcal{S}$. Therefore, there exists $c_{4}<1$ depending on $C_{1}$ such that

$$
\operatorname{dist}(x, \partial \Omega) \leq c_{4} \operatorname{dist}(x, \mathcal{S}) .
$$

We denote $r_{x}:=\operatorname{dist}(x, \mathcal{S})$. If

$$
\operatorname{dist}(x, \partial \Omega) \leq \min \left\{c_{4}, 10^{-1}\right\} r_{x},
$$

there exists $\xi_{x} \in \partial \Omega$ such that $\left|x-\xi_{x}\right| \operatorname{dist}(x, \partial \Omega)$. Then $B 9 r_{x} / 10\left(\xi_{x}\right) \subset B_{r_{x}}(x)$. We can apply Proposition 6.1 in $\Omega \cap B_{9 r_{x} / 10}\left(\xi_{x}\right)$. Since $x \in B_{r_{x} / 5}\left(\xi_{x}\right)$, there holds

$$
c_{5}^{-1} \frac{u_{F}(z)}{U_{F}(z)} \leq \frac{u_{F}(x)}{U_{F}(x)} \leq c_{5} \frac{u_{F}(z)}{U_{F}(z)} \quad \forall z \in B_{r_{x} / 5}\left(\xi_{x}\right) \cap \Omega .
$$

We can take in particular $z$ such that $\left|z-\xi_{x}\right|=r_{x} / 5$ and $\operatorname{dist}(z, \partial \Omega)=$ $\max \left\{\operatorname{dist}(t, \partial \Omega): t \in B_{r_{x} / 5}\left(\xi_{x}\right) \cap \Omega\right\}$. Since the distance from $z$ to $\mathcal{S}$ is comparable to dist $(z, \partial \Omega)$, there exist $n_{0} \in \mathbb{N}_{*}$ depending on the geometry of $\Omega$ and $n_{0}$ points $\left\{a_{j}\right\}$ with the properties that $\operatorname{dist}\left(a_{j}, \partial \Omega\right) \geq \operatorname{dist}(z, \partial \Omega), B_{r_{x} / 10}\left(a_{j}\right) \cap$ $B_{r_{x} / 10}\left(a_{j+1}\right) \neq \emptyset$ for $j=1, \ldots, n_{0}-1, a_{1}=z$ and $a_{n_{0}}$ have the property (i) above, that is there exists some $y \in \mathcal{S}$ and an isometry $\mathcal{R}_{y}$ such that $\mathcal{R}_{y}\left(\bar{C}_{0}\right) \cap B_{r_{0}}(y) \subset$ 
$\Omega \cup\{y\}$ and $\left(a_{n_{0}}-y\right) /\left|a_{n_{0}}-y\right| \in S_{1}$. By classical Harnack inequality (see Theorem 5.29 Step 3), there holds

$$
u_{F}\left(a_{j}\right) \geq c_{6} u_{F}\left(a_{j+1}\right) \quad \text { and } \quad U_{F}\left(a_{j}\right) \geq c_{6}^{-1} U_{F}\left(a_{j+1}\right)
$$

for some $c_{6}>1$ depending on $N, q$ and $\Omega$ via the cone $C_{0}$. Therefore

$$
U_{F}(x) \leq c_{5} c_{6}^{2 n_{0}} \frac{u_{F}\left(a_{n_{0}}\right)}{U_{F}\left(a_{n_{0}}\right)} u_{F}(x) \leq c_{7} u_{F}(x),
$$

which implies (5.97) from case (i) applied to $a_{n_{0}}$.

Finally, if (5.100) holds, but also

$$
\operatorname{dist}(x, \partial \Omega) \geq \min \left\{c_{4}, 10^{-1}\right\} r_{x},
$$

this means that dist $(x, \partial \Omega)$ is comparable to $r_{x}$. Then we can perform the same construction as in the case (5.101) holds, except that we consider balls $B_{\operatorname{dist}(x, \partial \Omega) / 4}\left(a_{j}\right)$ in order to connect $x$ to a point $a_{n_{0}}$ satisfying (i). The number $n_{0}$ is always independent of $u_{F}$. Thus we derive again estimate (5.97) provided dist $(x, \partial \Omega) \leq r_{0} / 2$. In order to prove that this holds in whole $\Omega$, we consider some $0<r_{1} \leq r_{0} / 2$ such that $\Omega_{r_{1}}^{\prime}:=\left\{x \in \Omega: \operatorname{dist}(x, \Omega)>r_{1}\right\}$ is connected. The function $v$ solution of

$$
\begin{cases}-\Delta v+v^{q}=0 & \text { in } \Omega_{r_{1}}^{\prime} \\ v=c_{1} u_{F} & \text { in } \partial \Omega_{r_{1}}^{\prime}\end{cases}
$$

is larger that $U_{F}$ in $\Omega_{r_{1}}^{\prime}$. Since $c_{1} u_{F}$ is a super solution, $v \leq c_{1} u_{F}$ in $\Omega_{r_{1}}^{\prime}$. This implies that (5.97) holds in $\Omega$.

Inequality (5.97) implies uniqueness by the same argument as in the proof of Theorem 5.29, Step 5.

\section{Boundary Harnack inequality}

In this section we prove the following:

Proposition 6.1. Assume $\Omega$ is a bounded Lipschitz domain, $A \subset \partial \Omega$ is relatively open and $q>1$. Let $\left(r_{0}, \lambda_{0}\right)$ be the Lipschitz characteristic of $\Omega$ (see Subsection 2.1).

Let $u_{i} \in C(\Omega \cup A), i=1,2$, be positive solutions of

$$
-\Delta u+u^{q}=0 \quad \text { in } \Omega
$$

such that $u_{2} \leq u_{1}$ and $u_{i}=0$ on $A$. Put $S=\partial \Omega \backslash A$ and $d(x, S)=\operatorname{dist}(x, S)$. Let $y \in A$ and let

$$
r:=\min \left(r_{0} / 8, \frac{1}{4} d(y, S)\right.
$$


so that

$$
\partial\left(B_{4 r}(y) \cap \Omega\right)=\left(\bar{B}_{4 r}(y) \cap \partial \Omega\right) \cup\left(\partial B_{4 r}(y) \cap \Omega\right) .
$$

Assume also that there exists a constant $c_{1}$ independent of $y$ such that

$$
u_{1}(z) \leq c_{1} u_{2}(z)
$$

for any $z \in \partial B_{3 r}(y) \cap \Omega$ such that $\operatorname{dist}(z, \partial \Omega) \geq \beta|z-y|$, then

$$
\begin{aligned}
& c^{-1} \frac{u_{1}(z)}{u_{1}\left(z^{\prime}\right)} \leq \frac{u_{2}(z)}{u_{2}\left(z^{\prime}\right)} \leq c \frac{u_{1}(z)}{u_{1}\left(z^{\prime}\right)} \quad \forall z, z^{\prime} \in B_{2 r}(y) \cap \Omega \\
& \text { such that }\left|z^{\prime}\right|=2 r \text {, dist }\left(z^{\prime}, \partial \Omega\right) \geq \beta\left|z^{\prime}-y\right|
\end{aligned}
$$

where the constant $c>0$ depends only on $N, q, \beta, c_{1}$ and the Lipschitz characteristic of $\Omega$. In particular

$$
u_{1}(z) \leq c u_{2}(z) \quad \forall z \in B_{2 r}(y) \cap \Omega
$$

Proof. Without loss of generality we assume that $y=0$. We can also assume that the truncated cone with vertex 0

$$
\Gamma:=\left\{\zeta \in \mathbb{R}^{N}: 0<|\zeta|<4 r, \operatorname{dist}(\zeta, \partial \Omega)>\beta|\zeta|\right\}
$$

is such that $\bar{\Gamma}$ is a compact subset of $\Omega \cup\{0\}$

Let $b=d(0, S)$ and put

$$
\tilde{u}_{i}(x)=b^{-\frac{2}{q-1}} u_{1}(x / b), \quad i=1,2 .
$$

Then $\tilde{u}_{i}$ has the same properties as $u_{i}$ when $\Omega$ is replaced by $\Omega^{b}=\frac{1}{b} \Omega, S$ by $S^{b}=\frac{1}{b} S, \Gamma$ by $\Gamma^{b}=\frac{1}{b} \Gamma$ and $r$ by $\delta=r / b$. Of course $d\left(0, S^{b}\right)=1$ so that

$$
\delta=\min \left(r_{0} /(8 b), 1 / 4\right) .
$$

The functions $\tilde{u}_{i}$ satisfy the equation

$$
-\Delta \tilde{u}_{i}+\tilde{u}_{i}^{q}=0 \text { in } B_{4 \delta}(0) \cap \Omega^{b}
$$

and $\tilde{u}_{i}=0$ on $B_{4 \delta}(0) \cap \partial \Omega^{b}$. Therefore, by the Keller-Osserman estimate,

$$
\tilde{u}_{i} \leq c(N, q) \delta^{-2 /(q-1)} \text { in } \bar{B}_{3 \delta}(0) \cap \Omega^{b} .
$$

If $a(x)=\tilde{u}_{1}^{q-1}$ then $\tilde{u}_{1}$ satisfies

$$
-\Delta \tilde{u}_{1}+a(x) \tilde{u}_{1}=0 \quad \text { in }\left(\frac{1}{b} \Omega\right) \cap B_{1}(0),
$$

and $a(\cdot)$ is bounded in $\bar{B}_{3 \delta}(0)$. 
Let $w$ be the solution of

$$
\begin{cases}-\Delta w+a(x) w=0 & \text { in } B_{3 \delta}(0) \cap \Omega^{b} \\ w=0 & \text { on } \bar{B}_{3 \delta}(0) \cap \frac{1}{b} \partial \Omega \\ w=\tilde{u}_{2} \chi_{\Gamma^{b}} & \text { on } \partial B_{3 \delta}(0) \cap \Omega^{b} .\end{cases}
$$

By applying the boundary Harnack principle in $B_{3 \delta}(0) \cap \Omega^{b}$ (using the slightly more general form derived in [2, Theorem 2.1]) we obtain

$$
c^{-1} \frac{\tilde{u}_{1}\left(\zeta^{\prime}\right)}{\tilde{u}_{1}(\zeta)} \leq \frac{w\left(\zeta^{\prime}\right)}{w(\zeta)} \leq c \frac{\tilde{u}_{1}\left(\zeta^{\prime}\right)}{\tilde{u}_{1}(\zeta)} \quad \forall \zeta, \zeta^{\prime} \in B_{2 \delta}(0) \cap \Omega^{b},
$$

where the constant $c$ depends only on the Lipschitz characteristic of $\Omega^{b}$ (which is $\left(r_{0} / b, \lambda_{0} b\right)$ and therefore "better" then that of $\Omega$ when $\left.b \leq 1\right)$. Notice that

$$
\tilde{u}_{i}(\zeta) \leq c_{2} \tilde{u}_{i}\left(\zeta^{\prime}\right) \quad \forall \zeta, \zeta^{\prime} \in \Gamma^{b} \text { s.t. } 2 \delta \leq|\zeta|,\left|\zeta^{\prime}\right| \leq 3 \delta
$$

by Harnack inequality. Since $a(x)$ is bounded, it follows by standard representation formula and Harnack inequality applied to $\tilde{u}_{2}$ that

$$
\begin{aligned}
\min \left\{w(x):|x|=\delta, x \in \Gamma^{b}\right\} & \geq c_{3}^{\prime} \min \left\{w(x):|x|=3 \delta, x \in \Gamma^{b}\right\} \\
& \geq c_{3} \max \left\{w(x):|x|=3 \delta, x \in \Gamma^{b}\right\},
\end{aligned}
$$

where the constants $c_{i}(i=1,2)$ depend on the opening of the cone and thus on the Lipschitz characteristic of $\Omega^{b}$. Since $w \leq \tilde{u}_{2}$ the above inequalities imply

$$
\tilde{u}_{1}(\zeta) \leq c \tilde{u}_{2}(\zeta) \frac{\tilde{u}_{1}\left(\zeta^{\prime}\right)}{w\left(\zeta^{\prime}\right)} \leq \frac{c}{c_{3}} \tilde{u}_{2}(\zeta) \frac{\tilde{u}_{1}\left(\zeta^{\prime}\right)}{\tilde{u}_{2}\left(\zeta^{\prime}\right)} \quad \forall \zeta \in B_{2 \delta}(0) \cap \Omega^{b}, \forall \zeta^{\prime} \in \partial B_{2 \delta}(0) \cap \Gamma^{b}
$$

In particular, it implies

$$
\tilde{u}_{1}(\zeta) \leq c^{\prime} \tilde{u}_{2}(\zeta) \quad \forall \zeta \in B_{2 \delta}(0) \cap \Omega^{b}
$$

This completes the proof.

\section{References}

[1] P. BARAS and M. PIERRE, Singularités éliminables pour des équations semi-lineaires, Ann. Inst. Fourier (Grenoble) 34 (1984), 185-206.

[2] S. BAUMAN, Positive solutions of elliptic equations in nondivergence form and their adjoints, Ark. Mat. 22 (1984), 153-173. 
[3] K. Bogdan, Sharp estimates for the Green function in Lipschitz domains, J. Math. Anal. Appl. 243 (2000), 326-337.

[4] H. BrezIS and F. BROWDER, Sur une propriété des espaces de Sobolev, C. R. Acad. Sci. Paris (A-B) 287 (1978), A113-A115.

[5] B. E. DalhberG, Estimates on harmonic measures, Arch. Ration. Mech. Anal. 65 (1977), 275-288.

[6] E. B. Dynkin, "Diffusions, Superdiffusions and Partial Differential Equations", Amer. Math. Soc. Colloquium Publications, 50, Providence, RI, 2002.

[7] E. B. Dynkin, "Superdiffusions and Positive Solutions of Nonlinear Partial Differential Equations", University Lecture Series, 34, Amer. Math. Soc., Providence, RI, 2004.

[8] E. B. DYNKIN and S. E. KuZNETSOv, Solutions of nonlinear differential equations on a Riemanian manifold and their trace on the boundary, Trans. Amer. Math. Soc. 350 (1998), 4217-4552.

[9] J. FABBRI and L. VÉRON, Singular boundary value problems for nonlinear elliptic equations in non smooth domains, Adv. Differential Equations 1 (1996), 1075-1098.

[10] N. Gilbarg and N. S. TRudinger, "Partial Differential Equations of Second Order", 2nd ed., Springer-Verlag, Berlin/New-York, 1983.

[11] A. GMIRA and L. VÉRON, Boundary singularities of solutions of nonlinear elliptic equations, Duke Math. J. 64 (1991), 271-324.

[12] R. A. Hunt and R. L. WhEeden, Positive harmonic functions on Lipschitz domains, Trans. Amer. Math. Soc. 147 (1970), 507-527.

[13] D. S. JERISON and C. E. KENIG, Boundary value problems on Lipschitz domains, Studies in partial differential equations, MAA Stud. Math. 23, (1982) 1-68.

[14] D. S. JERISON and C. E. KENIG, The Dirichlet problems in non-smooth domains, Ann. of Math. 113 (1981), 367-382.

[15] C. KENIG and J. PIPHER, The h-path distribution of conditioned Brownian motion for nonsmooth domains, Probab. Theory Related Fields 82 (1989), 615-623.

[16] J. B. Keller, On solutions of $\Delta u=f(u)$, Comm. Pure Appl. Math. 10 (1957), 503-510.

[17] J. F. LE GALL, The Brownian snake and solutions of $\Delta u=u^{2}$ in a domain, Probab. Theory Related Fields 102 (1995), 393-432.

[18] J. F. LE GALL, "Spatial Branching Processes, Random Snakes and Partial Differential Equations" Lectures in Mathematics ETH Zürich. Birkhäuser Verlag, Basel, 1999.

[19] M. MARCUS, Complete classification of the positive solutions of $-\Delta u+u^{q}=0$, J. d'Analyse Math., to appear.

[20] M. MARCUS and L. VÉRON, The boundary trace of positive solutions of semilinear elliptic equations: the subcritical case, Arch. Ration. Mech. An. 144 (1998), 201-231.

[21] M. MARCUS and L. VÉRON, The boundary trace of positive solutions of semilinear elliptic equations: the supercritical case, J. Math. Pures Appl. 77 (1998), 481-521.

[22] M. MARCUS and L. VÉRON, Removable singularities and boundary traces, J. Math. Pures Appl. 80 (2001), 879-900.

[23] M. MARCUS and L. VÉRON The boundary trace and generalized boundary value problem for semilinear elliptic equations with coercive absorption, Comm. Pure Appl. Math. (6) 56 (2003), 689-731.

[24] M. MARCUS and L. VÉRON, The precise boundary trace of positive solutions of the equation $\Delta u=u^{q}$ in the supercritical case, Perspectives in nonlinear partial differential equations, Contemp. Math., Amer. Math. Soc., Providence, RI 446 (2007), 345-383.

[25] M. MARCUS and L. VÉRON, Boundary trace of positive solutions of semilinear elliptic equations in Lipschitz domains, arXiv:0907.1006 (2009).

[26] B. MSELATI, Classification and probabilistic representation of the positive solutions of a semilinear elliptic equation, Mem. Amer. Math. Soc. 168, 798 (2004).

[27] R. Osserman, On the inequality $\Delta u \geq f(u)$, Pacific J. Math. 7 (1957), 1641-1647.

[28] N. TRUDINGER, On Harnack type inequalities and their application to quasilinear elliptic equations, Comm. Pure App. Math. 20 (1967), 721-747. 
[29] L. VÉRON, "Singularities of Solutions of Second Order Quasilinear Equations", Pitman Research Notes in Math. 353, Addison-Wesley-Longman, 1996.

Department of Mathematics

Technion Haifa, Israel

marcusm@math.technion.ac.il

Laboratoire de Mathématiques

Faculté des Sciences

Parc de Grandmont

37200 Tours, France

veronl@lmpt.univ-tours.fr 